\title{
Ionic currents influencing spontaneous firing and pacemaker frequency in dopamine neurons of the ventrolateral periaqueductal gray and dorsal raphe nucleus (vIPAG/DRN): A voltage-clamp and computational modelling study
}

\author{
Antonios G. Dougalis ${ }^{1,2,3}$ • Gillian A. C. Matthews ${ }^{1,2,4} \cdot$ Birgit Liss $^{3}$ • Mark A. Ungless ${ }^{1,2}$ \\ Received: 25 April 2014 /Revised: 28 October 2016 / Accepted: 13 March 2017 / Published online: 3 April 2017 \\ (C) The Author(s) 2017. This article is published with open access at Springerlink.com
}

\begin{abstract}
Dopamine (DA) neurons of the ventrolateral periaqueductal gray (vlPAG) and dorsal raphe nucleus (DRN) fire spontaneous action potentials (APs) at slow, regular patterns in vitro but a detailed account of their intrinsic membrane properties responsible for spontaneous firing is currently lacking. To resolve this, we performed a voltage-clamp electrophysiological study in brain slices to describe their major ionic currents and then constructed a computer model and used simulations to understand the mechanisms behind autorhythmicity in silico. We found that vlPAG/DRN DA neurons exhibit a number of voltage-dependent currents activating in the subthreshold range including, a hyperpolarization-activated cation current $\left(\mathrm{I}_{\mathrm{H}}\right)$, a transient, A-type, potassium current $\left(\mathrm{I}_{\mathrm{A}}\right)$, a background, 'persistent' $\left(\mathrm{I}_{\mathrm{NaP}}\right)$ sodium current and a transient, low voltage activated (LVA) calcium current $\left(\mathrm{I}_{\mathrm{CaLVA}}\right)$. Brain slice pharmacology, in good agreement with computer simulations, showed that spontaneous firing occurred independently of $\mathrm{I}_{\mathrm{H}}, \mathrm{I}_{\mathrm{A}}$ or
\end{abstract}

Action Editor: Charles Wilson

Electronic supplementary material The online version of this article (doi:10.1007/s10827-017-0641-0) contains supplementary material, which is available to authorized users.

Mark A. Ungless

mark.ungless@imperial.ac.uk

1 MRC London Institute of Medical Sciences (LMS), Du Cane Road, London W12 0NN, UK

2 Institute of Clinical Sciences (ICS), Imperial College London, Faculty of Medicine, Du Cane Road, London W12 0NN, UK

3 Institute of Applied Physiology, University of Ulm, Faculty of Medicine, 89073 Ulm, Germany

4 Present address: Picower Institute for Learning and Memory, Department of Brain and Cognitive Sciences, Massachusetts Institute of Technology, Cambridge, MA 02139, USA calcium currents. In contrast, when blocking sodium currents, spontaneous firing ceased and a stable, non-oscillating membrane potential below AP threshold was attained. Using the DA neuron model we further show that calcium currents exhibit little activation (compared to sodium) during the interspike interval (ISI) repolarization while, any individual potassium current alone, whose blockade positively modulated AP firing frequency, is not required for spontaneous firing. Instead, blockade of a number of potassium currents simultaneously is necessary to eliminate autorhythmicity. Repolarization during ISI is mediated initially via the deactivation of the delayed rectifier potassium current, while a sodium background 'persistent' current is essentially indispensable for autorhythmicity by driving repolarization towards AP threshold.

Keywords Autorhythmicity $\cdot$ Electrophysiology $\cdot$ Delayed rectifier $\cdot$ Persistent sodium current $\cdot$ Depolarization block

\section{Introduction}

Studies of physiological characteristics of dopamine (DA) neurons have been largely concentrated around the midbrain areas of substantia nigra pars compacta $(\mathrm{SNc})$ and ventral tegmental area (VTA) due to their well-known importance in motor control and reward processing (Schultz 1998; Pollack 2001). Midbrain DA neurons fire spontaneous action potentials (APs) at 3-8 Hz in a tonic or bursting manner in vivo (Grace and Bunney 1983a, 1983b; Grace and Bunney 1984a, 1984b) and maintain spontaneous tonic (but not bursting) firing at 1$10 \mathrm{~Hz}$ even in the absence of synaptic transmission in vitro (Grace and Onn 1989; Lacey et al. 1989; Puopolo et al. 2007; Lammel et al. 2008; Khaliq and Bean 2010). Through intensive research using the in vitro brain slice preparation and/or dissociated cell preparations a number of intrinsic ionic currents have 
been described to modulate firing frequency of midbrain DA neurons including, a hyperpolarization-activated cation current $\left[\mathrm{I}_{\mathrm{H}}\right]$ (Seutin et al. 2001; Neuhoff et al. 2002), an A-type potassium current $\left[\mathrm{I}_{\mathrm{A}}\right]$ (Liss et al. 2001; Koyama and Appel 2006a; Khaliq and Bean 2008; Kimm and Bean 2014; Subramaniam et al. 2014), an M-type potassium current $\left[\mathrm{I}_{\mathrm{M}}\right]$ (Hansen et al. 2006; Koyama and Appel 2006b; Koyama et al. 2007; Drion et al. 2010), an apamin-sensitive, calcium-activated potassium current $\left[I_{\text {SK }}\right]$ (Wolfart et al. 2001; Koyama et al. 2005; Bishop et al. 2010; Deignan et al. 2012), an iberiotoxin-sensitive calcium-activated potassium current $\left[\mathrm{I}_{\mathrm{BK}}\right]$ (Kimm et al. 2015), a delayed rectifier potassium current $\left[\mathrm{I}_{\mathrm{Kdr}}\right]$ (Silva et al. 1990; Kimm et al. 2015), transient (low voltage activated, LVA) and persistent (high voltage activated, HVA) calcium currents $\left[\mathrm{I}_{\mathrm{CaLVA}}\right.$ and $\left.\mathrm{I}_{\mathrm{CaHVA}}\right]$ (Kang and Kitai 1993a, 1993b; Wolfart and Roeper 2002; Branch et al. 2014; Poetschke et al. 2015; Philippart et al. 2016) and background 'persistent' and transient sodium currents $\left[\mathrm{I}_{\mathrm{NaP}}\right.$ and $\left.\mathrm{I}_{\mathrm{NaT}}\right]$ (Puopolo et al. 2007; Khaliq and Bean 2010; Ding et al. 2011).

The tonic-firing pacemaking mechanism of SNc and VTA midbrain DA neurons seem to differ in at least two important ways. First, $\mathrm{I}_{\mathrm{H}}$ current inhibition decreases pacemaker firing frequency in some SNc neurons but not in VTA neurons (Neuhoff et al. 2002; Khaliq and Bean 2010) arguing that $I_{H}$ current may have a role in pacemaking in $\mathrm{SNc}$ neurons but it is not essential for autorhythmicity in either group. It is noteworthy that VTA neurons, especially those projecting to the prefrontal cortex, lack a prominent expression of an $\mathrm{I}_{\mathrm{H}}$ current (Margolis et al. 2006; Lammel et al. 2008). Second, during the interspike interval (ISI), SNc neurons rely substantially on subthreshold calcium conductances to repolarise to AP threshold (Wilson and Callaway 2000; Chan et al. 2007; Puopolo et al. 2007; Putzier et al. 2009) and to a far lesser degree on sodium conductances (Puopolo et al. 2007; Khaliq and Bean 2010). Interestingly, the opposite is true for VTA neurons that, rely heavily on both voltage-dependent, TTX-sensitive and voltage-independent, TTX-resistant, background 'persistent' sodium currents to repolarise to AP threshold during ISI (Khaliq and Bean 2010). SNc pacemaker firing frequency can be often greatly reduced or even halted by L-type calcium channel blockers (Nedergaard et al. 1993; Mercuri et al. 1994; Puopolo et al. 2007; Putzier et al. 2009, but see also Chan et al. 2007, Guzman et al. 2009, Poetschke et al. 2015) or abolished by cadmium (Puopolo et al. 2007) suggesting that calcium currents are indispensable for SNc autorhythmicity although the impact of L-type blockers on firing rate of SNc DA neurons is often hard to reproduce for reasons that are not clear (see Chan et al. 2007; Guzman et al. 2009; see also explanations provided in modeling study by Drion et al. 2011). In contrast, in VTA neurons, the absence of external calcium speeds rather than abolishes the pacemaker firing frequency (Khaliq and Bean 2010), whereas blockade of a TTXsensitive sodium current resulted in a stable resting membrane potential (negative to AP threshold) with no evidence of background oscillations, a situation unlike to what has been reported for SNc neurons that exhibit calcium-mediated small oscillating potentials (SOP) in the presence of sodium channel blockers (Nedergaard et al. 1993; Mercuri et al. 1994; Chan et al. 2007; Puopolo et al. 2007; Guzman et al. 2009). The presumed qualitative difference in the mechanism of pacemaking in VTA neurons and SNc neurons, in that there is little role of subthreshold calcium current in driving pacemaking in VTA neurons, also supports the hypothesis that the selective vulnerability of SNc DA neurons in Parkinson's disease (as opposed to the selective sparring of VTA neurons, see Mosharov et al. 2009; Surmeier and Schumacker 2013) may depend on this prominent calcium entry during pacemaking cycles and its link to mitochondrial potential flickering and oxidative stress (Guzman et al. 2010; Surmeier et al. 2011; Philippart et al. 2016). Given the diversity of the functional phenotypes of midbrain DA neurons based on their neuroanatomical positioning and projection targets (Lammel et al. 2008, 2011; Poulin et al. 2014; Beier et al. 2015; Lerner et al. 2015), it is likely that the VTA and SNc DA neuronal subgroups may utilise a multitude of mechanisms under different circumstances to maintain tonic firing (e.g. see results in Chan et al. 2007; Puopolo et al. 2007; Guzman et al. 2009; Poetschke et al. 2015) which in turn could influence to a different degree the neuron's propensity for degeneration.

The ventrolateral periaqueductal grey (vlPAG) and dorsal raphe nucleus (DRN) regions contain a population of DA neurons, often considered to be a dorsocaudal extension of the VTA area towards the brainstem, termed collectively the A10dc system (Hokfelt et al. 1984). These DA neurons may play a role in wakefulness ( $\mathrm{Lu}$ et al. 2006), opiate antinociception (Flores et al. 2004; Li et al. 2016), drug reward (Flores et al. 2006; Li et al. 2013) while they have been recently shown to encode the experience of social isolation (Matthews et al. 2016). Using the pitx3-GFP and TH-GFP transgenic mouse models as an aid to identification of DA neuron phenotype, we have previously reported the physiological characteristics of DA vlPAG/DRN neurons in vitro under current-clamp (regular firing with broad APs at a range $1-10 \mathrm{~Hz}$ and spike-frequency adaptation in response to prolonged depolarization, Dougalis et al. 2012). Interestingly during our studies we have also noted that spontaneous tonic firing of DA vlPAG/DRN neurons in vitro can persist in the absence of glutamatergic and GABAergic synaptic transmission but the mechanism behind this property is not known despite the availability of such data for SNc and VTA, midbrain DA neuronal groups (see Grace and Onn 1989; Silva et al. 1990; Puopolo et al. 2007; Khaliq and Bean 2010). To resolve this, we have conducted a voltage-clamp study to provide a kinetic description of major sodium, potassium and calcium ionic currents operant on DA vlPAG/DRN neurons in brain slices. Based on experimentally derived voltage- 
clamp data, we then constructed a simplified, conductancebased, Hodgkin and Huxley-type, computer model and validated its behaviour against in vitro neurophysiological data. Using simulations in the computational DA model, we explored the contribution of individual ionic currents in vlPAG/DRN DA neuron's spontaneous firing, pacemaker frequency and threshold for spike frequency adaptation in silico.

\section{Methods}

\subsection{Brain slice preparation}

Three to six months old, male, GFP-pitx3 heterozygous mice (transgenic line generated as described previously, see Zhao et al. 2004) were sacrificed by isoflurane anaesthesia followed by decapitation. All breeding and experimental procedures were conducted in accordance with the Animals (Scientific Procedures) Act of 1986 (United Kingdom) or were approved by the German Regierungspräsidium Tübingen (AZ 35/ 9185.81-3. TV1043, Reg. Nr. 0.147). The brain was rapidly removed out of the cranial cavity and bathed with ice-cold (0$2{ }^{\circ} \mathrm{C}$ ), fully equilibrated (with carbogen gas, $95 \%$ carbon dioxide and 5\% oxygen) artificial celebrospinal fluid (aCSF, composition in mM, NaCl 120, $\mathrm{KCl} 3.5, \mathrm{NaH}_{2} \mathrm{PO}_{4} 1.25$, $\mathrm{NaHCO}_{3} 26$, Glucose $10, \mathrm{MgCl}_{2} 1, \mathrm{CaCl}_{2}$ 2). Two or three thin coronal brain slices ( $220 \mu \mathrm{m}$ thickness) encompassing the dorsal raphe nucleus (DRN) were obtained using a vibratome (Leica VT1000S, Germany) and were maintained into a standard in-house-made maintenance chamber (Edwards et al. 1989) gently and continuously aerated with carbogen gas for at least one and a half hours at room temperature $\left(20-22^{\circ} \mathrm{C}\right)$ before use for electrophysiology.

\subsection{Neuron identification}

Slices were transferred to a submersion recording chamber and were continuously perfused at a rate of $2-4 \mathrm{ml} / \mathrm{min}$ with fully oxygenated aCSF (composition as above) at $35.5^{\circ} \mathrm{C}( \pm$ $0.5^{\circ} \mathrm{C}$ ) for at least $30 \mathrm{~min}$ before attempting recordings. Whole-cell current and voltage-clamp recordings were obtained from DA neurons using infra-red differential interference contract (IR-DIC) videomicroscopy as described previously (Stuart et al. 1993). In short, neurons were visualized under an upright microscope equipped with $\times 40$ or $\times 60$ objectives, an IR filter, DIC optics and a charged cooled diode (CCD) video camera. GFP-pitx3 DA neurons were firstly identified in the slice by using fluorescence illumination coupled to a GFP excitation filter and then further visualized under IR-DIC conditions prior to electrophysiology. For comparative purposes, in some experiments, we also obtained targeted recordings from SNc and VTA DA neurons as described previously (Bishop et al. 2010).

\subsection{Electrophysiology}

Tight seal (>10 G $\Omega$ ) whole-cell, current-clamp recordings were performed with a Multiclamp 700B amplifier (Molecular Devices), while voltage-clamp recordings were performed with an Axopatch 200A (Molecular Devices) or an EPC-10 (HEKA Electronics, Germany) using glass microelectrodes (3-6 M $\Omega$ in resistance) filled with a standard internal solution containing (in $\mathrm{mM}$ ) 140 potassium gluconate (KGlu), $5 \mathrm{NaCl}, 1 \mathrm{MgCl}_{2}$, 10 HEPES, 1 EGTA, 2 MgATP and 0.5 LiGTP, pH 7.25-7.35, osmolality 280-290 mosmol/1. For voltage-clamp recordings aiming to isolate specific ionic currents, internal and external solutions were modified accordingly depending on the current under investigation (see descriptive text below in solutions and voltage-clamp protocols for ionic currents). Series resistance $\left(\mathrm{R}_{\mathrm{s}}\right)$ and input resistance $\left(\mathrm{R}_{\text {in }}\right)$ were frequently monitored throughout the experiments via at $10 \mathrm{mV}, 250 \mathrm{~ms}$ hyperpolarizing step under voltageclamp. $\mathrm{R}_{\mathrm{s}}$ value was $<13 \mathrm{M} \Omega$ (average $8.3 \pm 1.1 \mathrm{M} \Omega$, $n=40$ ) for all recordings and was compensated by approximately $60-90 \%$ leaving on average an uncompensated Rs of 3.2 $\mathrm{M} \Omega$ (range, 2.5-7 $\mathrm{M} \Omega$ ) and an estimated error in voltageclamp command potentials when recording an one nanoampere current under most circumstances of $<5 \mathrm{mV}$. Whole-cell capacitive transients were evaluated under voltage-clamp through a $10 \mathrm{mV}$ step $(-50$ to $-60 \mathrm{mV})$ and were negated using the amplifier's circuitry. The recorded neurons had an average $\mathrm{C}_{\mathrm{W}}$ of $7.8 \pm 0.4 \mathrm{pF}$ ( $n=40$, range, 4.5 to $12.0 \mathrm{pF}$ ). Currents were filtered at $1 \mathrm{KHz}$ (low-pass), collected at $3-5 \mathrm{KHz}$ through an $\mathrm{A} / \mathrm{D}$ converter (PC-5230, National Instruments, USA) on WINWCP (University of Strathclyde, courtesy of Dr. John Dempster) or via an onboard card from EPC-10 to PatchMaster software (HEKA Electronics, Germany) and stored for offline analysis. The combination of our intracellular and extracellular recording solutions lead to an estimated liquid junction potential (LJP) of +7 and $+12 \mathrm{mV}$ for the cesium methanesulphonate (CsMe) and KGlu filled electrodes respectively (measured in pClamp10 calculator). Holding voltages reported herein for electrophysiological experiments have been corrected by this amount.

\subsection{Solutions and pharmacology}

The standard KGlu internal solution (detailed above) was used in voltage-clamp experiments isolating sustained $\left(\mathrm{I}_{\mathrm{Kdr}}\right.$ and $\left.\mathrm{I}_{\mathrm{M}}\right)$ and transient $\left(\mathrm{I}_{\mathrm{A}}\right)$ potassium currents as well as the hyperpolarization-activated cation current $\left(\mathrm{I}_{\mathrm{H}}\right)$. In these experiments the aCSF was supplemented with tetrodotoxin (TTX, $1 \mu \mathrm{M})$. For experiments involving calcium, barium and sodium current isolation, KGlu was substituted for $\mathrm{CsMe}$ $(120 \mathrm{mM})$ and tetraethylammonium (TEA, $20 \mathrm{mM}$ ) in internal solutions. External aCSF solution's $\mathrm{NaCl}$ concentration was 
reduced to $100 \mathrm{mM}$ and was supplemented with $20 \mathrm{mM}$ TEA, $2 \mathrm{mM}$ 4-aminopyridine (4-AP) and $1 \mu \mathrm{M}$ TTX for calcium/ barium current recordings. Barium chloride $\left(\mathrm{BaCl}_{2}\right)$ substituted calcium chloride in the external solution for barium current recordings ( $1 \mathrm{mM} \mathrm{BaCl}_{2}$ for $2 \mathrm{mM} \mathrm{CaCl}_{2}$ ). For isolation of sodium currents we included $1 \mathrm{mM} \mathrm{BaCl}_{2}$ in external aCSF and replaced TTX with cadmium chloride $(200 \mu \mathrm{M})$. Osmolality in all above aCSF solutions was kept constant at $300 \mathrm{mosmol} / \mathrm{l}$ by equivalent reductions in $\mathrm{NaCl}$ when more than $2 \mathrm{mM}$ additions were made. All drugs used in this study for formulating external and internal solutions were bought from Sigma-Aldrich (UK) apart from 2,3dihydroxy-6-nitro-7-sulfamoyl-benzo[f] quinoxaline-2,3dione (NBQX), tetrodotoxin (TTX), 10,10-bis(4Pyridinylmethyl)-9(10H)-anthracenone dihydrochloride (XE991) and 4-Ethylphenylamino-1,2-dimethyl-6ethylaminopyrimidinine (ZD7288) which were bought from Tocris (UK). For pharmacological investigations, drugs were made fresh from appropriately concentrated aliquots stored according to the manufacturer's recommendations. All drugs were dissolved on the day in freshly prepared oxygenated aCSF and superfused on the tissue for $10 \mathrm{~min}$ to achieve steady-state concentration in the intima of the brain slices before any measurements were taken.

\subsection{Voltage-clamp protocols for ionic currents}

\subsubsection{Hyperpolarization-activated cation current $\left(I_{H}\right)$}

Neurons were voltage-clamped at $-47 \mathrm{mV}$ and hyperpolarizing steps of $1 \mathrm{~s}$ in duration were delivered in $10 \mathrm{mV}$ decrements from -62 to $-152 \mathrm{mV}$ in the presence of TTX $(1 \mu \mathrm{M})$ using a standard KGlu based-internal solution. Determination of $\mathrm{I}_{\mathrm{H}}$ current amplitude at potentials more negative than $-152 \mathrm{mV}$ was not routinely attempted as this lead to loss of recording. $\mathrm{I}_{\mathrm{H}}$ currents were measured as slowly activating inward currents calculated from the difference of the instantaneous currents and the steady-state currents, in the first 5$20 \mathrm{~ms}$ after the beginning and $25 \mathrm{~ms}$ before the end of the hyperpolarizing voltage step respectively, at each test holding potential.

\subsubsection{A-type $\left(I_{A}\right)$ and delayed rectifier $\left(I_{K d r}\right)$ potassium currents}

$\mathrm{I}_{\mathrm{A}}$ currents were isolated by digital subtraction of currents between two specific voltage-clamp protocols using a standard KGlu based-internal solution in the presence of TTX $(1 \mu \mathrm{M})$ as reported previously (Koyama and Appel 2006a). $\mathrm{I}_{\mathrm{A}}$ current amplitude was measured from peak till $25 \mathrm{~ms}$ before the end of the inactivating current during the $1 \mathrm{~s}$ long pulses used in our study. To study $\mathrm{I}_{\mathrm{A}}$ current steady-state activation, neurons were held at $-72 \mathrm{mV}$ and a $250 \mathrm{~ms}$ hyperpolarizing prepulse to $-112 \mathrm{mV}$ was used to facilitate the recovery from inactivation of the $\mathrm{I}_{\mathrm{A}}$ current. Subsequently the neurons were stepped to the test voltage from -92 to $+18 \mathrm{mV}$ in $10 \mathrm{mV}$ increments for $1 \mathrm{~s}$ to activate and record the resultant $\mathrm{I}_{\mathrm{A}}$ current transient and its inactivation (protocol 1). In the second protocol, neurons were held at $-72 \mathrm{mV}$ and a $250 \mathrm{~ms}$ prepulse to $-52 \mathrm{mV}$ was given to facilitate the inactivation of the $\mathrm{I}_{\mathrm{A}}$ transient current before stepping the neurons from -92 to $+18 \mathrm{mV}$ in $10 \mathrm{mV}$ increments for $1 \mathrm{~s} \mathrm{(protocol} \mathrm{2).}$ Currents obtained from the second protocol were slowly activating sustained outward currents reminiscent of a delayed rectifier $\left(\mathrm{I}_{\mathrm{Kdr}}\right)$ and were subtracted from the currents obtained from protocol 1 to obtain the $\mathrm{I}_{\mathrm{A}}$ current. To study $\mathrm{I}_{\mathrm{A}}$ current steady-state inactivation, neurons were held at $-47 \mathrm{mV}$ and a $250 \mathrm{~ms}$ prepulse was given from $-152 \mathrm{mV}$ to $-52 \mathrm{mV}$ (in $10 \mathrm{mV}$ increments) before stepping the neuron to the test voltage of $-47 \mathrm{mV}$ for $1 \mathrm{~s}$ to record the extend of $\mathrm{I}_{\mathrm{A}}$ current inactivation as a function of the prepulse holding voltage. Current subtraction was not necessary for steady-state inactivation experiments because the fast peak of $\mathrm{I}_{\mathrm{A}}$ current was not contaminated by the slowly activating sustained currents at the test voltage of $-47 \mathrm{mV}$. We also obtained $\mathrm{I}_{\mathrm{A}}$ currents via a single activation voltage protocol (protocol 1 described above) in the presence of $10 \mathrm{mM}$ TEA (to block $\mathrm{I}_{\mathrm{Kdr}}$ potassium currents) as described previously (Silva et al. 1990) to compare the results obtained via the two protocol subtraction method. Finally, $\mathrm{I}_{\mathrm{A}}$ currents were also isolated via a third method using protocol 1 above in the presence of TEA $(10 \mathrm{mM})$ by current subtraction after the addition of $2 \mathrm{mM}$ 4-AP to reveal the 4-AP sensitive component. $\mathrm{I}_{\mathrm{Kdr}}$ currents were isolated using a KGlu based-internal solution in the presence of TTX $(1 \mu \mathrm{M})$ as reported previously (Silva et al. 1990) using a modified protocol 2 detailed above. To study $\mathrm{I}_{\mathrm{Kdr}}$ current steady-state activation neurons were held at $-72 \mathrm{mV}$ and a $250 \mathrm{~ms}$ hyperpolarizing prepulse to $-52 \mathrm{mV}$ was used to facilitate the inactivation of the $\mathrm{I}_{\mathrm{A}}$ transient current. Subsequently the neurons were stepped to the test voltage from -92 to $+18 \mathrm{mV}$ in $10 \mathrm{mV}$ increments for $1 \mathrm{~s}$ to activate and record the resultant slowly-activating sustained $\mathrm{I}_{\mathrm{Kdr}}$ current. Current amplitude was measured $25 \mathrm{~ms}$ before the end of the depolarizing step.

\subsubsection{M-type $\left(I_{M}\right)$ potassium currents}

$\mathrm{I}_{\mathrm{M}}$ currents were isolated using a standard deactivation protocol in KGlu-based internal solution in the presence of TTX $(1 \mu \mathrm{M})$ as reported previously (Koyama and Appel 2006b). The neurons were held at $-72 \mathrm{mV}$ and were given a depolarizing prepulse to $-32 \mathrm{mV}$ for $1 \mathrm{~s}$ before stepped down to the test voltage from -42 to $-72 \mathrm{mV}$ in $10 \mathrm{mV}$ decrements for $1 \mathrm{~s}$ to record the resultant $\mathrm{I}_{\mathrm{M}}$ current deactivation tail. 


\subsubsection{Calcium and Barium currents $\left(I_{C a}\right.$ and $\left.I_{B a}\right)$}

Low and high threshold voltage-activated (LVA and HVA) inward calcium/barium currents (LVA and HVA) were isolated using a combination of blockers in both internal and external solutions in an attempt to block potassium and sodium conductances as described previously (Brevi et al. 2001). Neurons were bathed in TTX $(1 \mu \mathrm{M}), 4-\mathrm{AP}(2 \mathrm{mM})$ and TEA $(20 \mathrm{mM})$ and recorded with CsMe $(120 \mathrm{mM})$ and TEA $(20 \mathrm{mM})$ based internal solutions. For $\mathrm{I}_{\mathrm{Ca}}$ experiments, neurons were held at $-67 \mathrm{mV}$ and were depolarized to the test voltage from -57 to $+3 \mathrm{mV}$ in $10 \mathrm{mV}$ increments for $250 \mathrm{~ms}$. Calcium currents recorded through this protocol would inactivate with different time constants during our steps at nearly all potentials. This inactivation could theoretically result from calcium-mediated intracellular calcium release (that can induce calcium channel inactivation) or it could be directly related to the kinetic, voltage-dependent properties of the calcium channels (Giannattasio et al. 1991; Haack and Rosenberg 1994; Catterall 2000; Budde et al. 2002). To reduce the amount of inactivation seen with calcium and discriminate better between these two possibilities we have used barium ions $(1 \mathrm{mM})$ instead of calcium $(2 \mathrm{mM})$ as the charge carrier, since barium currents undergo significantly less inactivation during long steps than calcium currents (see Hille 2001). Furthermore, to ascertain the presence of LVA currents we recorded barium currents from a more hyperpolarized potential $(-87 \mathrm{mV}) . \mathrm{I}_{\mathrm{Ba}}$ were recorded via two different protocols essentially used to isolate LVA/HVA or HVA currents alone (Brevi et al. 2001). Neurons were held at either $-87 \mathrm{mV}$ (LVA/ HVA protocol) or $-67 \mathrm{mV}$ (HVA protocol) and were depolarized in $10 \mathrm{mV}$ increments for $250 \mathrm{~ms}$ to $+3 \mathrm{mV}$. Current subtraction was used to isolate the LVA component from LVA/HVA protocol by subtracting the HVA protocol currents. As an alternative method to study calcium and barium currents, we employed fast voltageramps $(200-500 \mathrm{mV} / \mathrm{s}$, from -107 to $+13 \mathrm{mV})$ to measure the background flowing currents at different potentials after leak current subtraction.

\subsubsection{Sodium currents $\left(I_{N a}\right)$}

Fast transient $\left(\mathrm{I}_{\mathrm{NaT}}\right)$ and persistent $\left(\mathrm{I}_{\mathrm{NaP}}\right)$ sodium currents were recorded from neurons bathed in TEA $(20 \mathrm{mM})$, barium chloride $(1 \mathrm{mM})$ and cadmium chloride $(200 \mu \mathrm{M})$ using CsMe/ TEA $(120 / 20 \mathrm{mM})$-based internal solutions as reported previously (Pignatelli et al. 2005; Magistretti et al. 2006). Neurons were held at $-107 \mathrm{mV}$ and a depolarizing prepulse of $5 \mathrm{~ms}$ in duration was delivered to $-47 \mathrm{mV}$ to activate unclamped axonal sodium currents, before hyperpolarizing the neuron to $-77 \mathrm{mV}$ for $4 \mathrm{~ms}$ and then step wise depolarizing it (in $5 \mathrm{mV}$ increments from -77 to $+13 \mathrm{mV}$ for $110 \mathrm{~ms}$ ) to sample somatic $\mathrm{I}_{\mathrm{Na}}$ current activation devoid of axonal contamination as described previously (Milescu et al. 2010). To study steadystate inactivation of transient somatic sodium currents, neurons were subjected to the above protocol with a prepulse to -47 to activate and inactivate axonal sodium currents but then were given a $4 \mathrm{~ms}$ variable hyperpolarizing step from -77 to $-42 \mathrm{mV}$ (in $5 \mathrm{mV}$ steps) before measuring the somatic sodium current at the test potential of $-37 \mathrm{mV}$ (held for $20 \mathrm{~ms}$ ). The amplitude of the background 'persistent' sodium current was measured $10 \mathrm{~ms}$ before the end of the depolarizing steps and of the transient sodium current at the peak of the response during the steady state activation/ inactivation protocol. We also sampled persistent noninactivating sodium currents via an alternative method by subjecting neurons to slow voltage ramps $(16 \mathrm{mV} / \mathrm{s}$, from -107 to $+53 \mathrm{mV}$ ) and leak current substraction in the presence of the above blocking solutions.

\subsection{Current-clamp experiments}

The standard KGlu internal solution and standard external aCSF (as detailed above) were used in all current-clamp experiments unless otherwise indicated in text. Experiments were conducted in the presence of NBQX $(5 \mu \mathrm{M})$ and picrotoxin $(25 \mu \mathrm{M})$ to block excitatory and inhibitory synaptic activity respectively.

\subsection{Voltage-clamp data analysis}

Voltage-clamped currents were averaged in three to five trials at each test holding voltage. We employed capacitive transient artifact and leak subtraction for sodium and calcium inward conductance using a modified $\mathrm{P} / 4$ protocol off-line. Averaged currents from different protocols were exported as text files (.txt) for subtraction in Excel spreadsheets and were then imported into Spike2 (Cambridge electronic design, CED, UK) for measurements and exponential function fitting. Data transformations, statistical analysis and fitting of single order Boltzmann function to the data set were performed in Prism software (version 7, GraphPad, USA).

Measured current amplitude at peak (or steady-state) was converted into conductance at any given holding potential by using eq. (1) below

$G=\frac{I}{V-\text { Erev }}$

where, $\mathrm{G}$ represents the current conductance at a given test holding voltage $\mathrm{V}$, while I is the current amplitude at the given holding voltage and $\mathrm{E}_{\mathrm{rev}}$ is the equilibrium potential (calculated using the Nernst equation) of the current under investigation. The Nernst equation yielded for our conditions $\left(35^{\circ} \mathrm{C}\right)$ an estimated equilibrium potential of $-105,+135,+75 \mathrm{mV}$ for the major ions $\left(\mathrm{E}_{\mathrm{K}+}, \mathrm{E}_{\mathrm{Ca}++}, \mathrm{E}_{\mathrm{Na}+}\right.$, respectively). 
To construct steady-state activation (or inactivation) curves we normalized conductance to its maximal value and plotted it against holding voltage $\left(\mathrm{G} / \mathrm{G}_{\max }\right.$ against holding voltage). The data were then fitted with a single first order Boltzmann function using eq. (2) below

$$
G=\frac{G \max +G \min }{1+\exp \left(\frac{V 50-V}{s}\right)}+G \min
$$

where, $\mathrm{G}$ is the conductance at the holding test voltage of $\mathrm{V}$, $\mathrm{G}_{\max }$ is the maximal conductance set to be 1 and $\mathrm{G}_{\min }$ is the minimum conductance set to be $0, V_{50}$ is the membrane potential for half-maximal activation and $\mathrm{s}$ is the slope factor of the activation/inactivation curve.

To measure the activation and inactivation time constants currents were fitted from start to peak (activation time constant) or from peak to end (inactivation time constant) with a single exponential function according to the eq. (3) below

$I=\operatorname{Imax}{ }^{*} \exp (-t / \tau)$

where, $\mathrm{I}$ is the current amplitude at time $\mathrm{t}$ and $\mathrm{I}_{\max }$ is the maximal current amplitude, while $\tau$ is the time constant. In some currents (e.g. sodium currents and A-type potassium currents), inactivation was best described by two exponential function fitting at most holding voltages. These currents were fitted accordingly with following eq. (4) for bi-exponential inactivation

$I=\operatorname{Imax}(\mathrm{f})^{*} \exp (-t / \tau f)+\operatorname{Imax}(\mathrm{s})^{*} \exp (-t / \tau s)$

where, I is the total current amplitude at time $t, I_{\max (f)}$ is the maximal current amplitude of the first (fast) inactivating component of the current and $\mathrm{I}_{\max (\mathrm{s})}$ is the maximal current amplitude of the second (slow) inactivating component of the current, while $\tau_{\mathrm{f}}$ and $\tau_{\mathrm{s}}$ are the fast and the slow component inactivation time constants respectively. Decision on using mono versus biexponential fitting was guided by inspection of the residual value table and compared by using the $\mathrm{R}$ value and the standard deviation for the fit. Calculated time constants were plotted against holding voltage and were fitted a single order modified first order Boltzmann function [eq. (5)] to calculate the voltage to attain half-maximal activation/inactivation time constant $\left(\mathrm{V}_{50}\right.$ value $)$ and its corresponding slope factor.

$\tau=\frac{\tau \max -\tau \min }{1+\exp \left(\frac{V 50-V}{s}\right)}+\tau \min$

where, $\tau$ is the activation/inactivation time constant at the holding test voltage of $\mathrm{V}, \tau_{\max }$ is the maximal value of the time constant, $\tau_{\min }$ is the minimum value of the time constant, $\mathrm{V}_{50}$ is the membrane potential where time constant is half maximal and $\mathrm{s}$ is the slope factor of the time constant curve.

\subsection{Current-clamp data analysis}

All neurons were monitored frequently for stability of series resistance $\left(\mathrm{R}_{\mathrm{s}}\right)$ and input resistance through the recording and during pharmacological investigations. $\mathrm{R}_{\mathrm{S}}$ did not exceed 25 $\mathrm{M} \Omega$ in our recordings. Action potentials (APs) and accompanying afterhyperpolarization (AHP) were averaged during epochs of interest (100-200 APs) in Spike2 software (CED, UK) and their characteristics were calculated (AP amplitude, AP width, AHP amplitude, AHP repolarization) with respect to the AP threshold (defined as the point where the rate of voltage change exceeded $10 \mathrm{mV} / \mathrm{ms}$, calculated through the first differential of the voltage trace). Average firing rate for each cell was calculated from the inverse of the average interspike interval (ISI) from ISI histograms while the coefficient of variation of the ISI was calculated as the ratio of the standard deviation (SD) of the ISI to the mean ISI. Hyperpolarizing current injections $(-10$ to $-120 \mathrm{pA}$, $1000 \mathrm{~ms}$ in duration) were used to ascertain the extent of $\mathrm{I}_{\mathrm{H}}$ current activation (referred to as the $\mathrm{I}_{\mathrm{H}}$ current-mediated voltage-sag) and of the extent of $\mathrm{I}_{\mathrm{A}}$ current activation (referred to as delayed repolarization). The voltage-sag was measured during the hyperpolarizing step as the voltage difference from the peak of the voltage response at the beginning of the step (occurring in the first 50-100 ms from step initiation) till $20 \mathrm{~ms}$ before the end of the hyperpolarizing step. Delayed repolarization was measured at the termination of the hyperpolarizing step as the time from step termination till the firing of the first AP. Depolarizing current injections ( +10 to $+120 \mathrm{pA}, 1000 \mathrm{~ms}$ in duration) were used to elicit APs and measure the gain (input-output relationship) of vlPAG/DRN DA neurons. We measured instantaneous firing frequency by using the first two APs elicited by the depolarizing step. Sustained firing frequency was measured as the mean AP firing frequency during the last $100 \mathrm{~ms}$ of the depolarizing step as described previously (Dougalis et al. 2012).

\subsection{Statistical data analysis}

All values reported represent mean \pm standard error of the mean (s.e.m). The $\mathrm{n}$ number reported for each experiment represents replicated observations in different neurons from slices obtained from a minimum of three different animals (range of 3-5 per experiment as indicated in results section for each experiment). A $P$ value of less than 0.05 was taken to indicate significance using a paired or unpaired t-test as appropriate for pair-wise comparisons or one-way analysis of variance (ANOVA) followed by Tukey's multiple comparison test. 


\subsection{Modelling and computer simulations}

The DA neuron was modelled using NEURON software (Hines and Carnevale 1997, available freely at https://www. neuron.yale.edu/neuron/) as a single compartment, spherical neuron of $15 \mu \mathrm{m}$ in diameter. General model assumptions and specific model equations used in the construction of the model are detailed in the appendix. The model code will be deposited and will be freely available through the modelDB database (https://senselab.med.yale.edu/modeldb/) following article publication (also available through direct request to the authors). Electrophysiological data were collected at $35{ }^{\circ} \mathrm{C}$ and thus the model replicates behaviour at this temperature. The model DA neuron was constructed to operate through eight Hodgkin and Huxley-type conductances $\left(\mathrm{I}_{\mathrm{NaT}}, \mathrm{I}_{\mathrm{NaP}}\right.$, $\mathrm{I}_{\mathrm{Kdr}}, \mathrm{I}_{\mathrm{A}}, \mathrm{I}_{\mathrm{M}}, \mathrm{I}_{\mathrm{H}}, \mathrm{I}_{\mathrm{CaHVA}}, \mathrm{I}_{\mathrm{CaLVA}}$ ) and a leak conductance. Since it is not possible to examine electrophysiologically a single neuron for all conductances, the values derived for any conductance represent mean values over a population of different neurons examined and these were used in the model unless otherwise stated. All values used in the model (Table 1) were mean values taken from experimental data. In some cases, values were optimized by allowing use of mean \pm one standard deviation of the reported experimental values where necessary to improve model functionality. For the operation of the model and in line with Hodgkin and Huxley (1952) we have used three activation gates for sodium currents and four activation gates for the delayed rectifier potassium current. Only one activation gate was used for calcium currents, $\mathrm{I}_{\mathrm{A}}$ current, $\mathrm{I}_{\mathrm{M}}$ and $\mathrm{I}_{\mathrm{H}}$ current as described previously (Xiao et al. 2004) and in line with our experimental fitting. Barium currents were shifted $10 \mathrm{mV}$ more positive to mirror the $\mathrm{V}_{50}$ values for calcium currents. The leak current in the DA model was given a constant conductance of $0.04 \mathrm{mS} / \mathrm{cm}^{2}$ and an $\mathrm{E}_{\text {rev }}$ of $-55 \mathrm{mV}$ based on experimental data of DA neurons mean input resistance $(7 \mathrm{G} \Omega)$ and reversal potential in the presence of calcium, sodium and potassium channel blockers. Simulated responses (numerical integration in steps of $25 \mu \mathrm{s}$ corresponding to a reconstruction frequency of $40 \mathrm{KHz}$ ) for the model DA neuron were exported programmatically from NEURON as text files (.txt) and imported in Spike2 (CED, UK) for measurements of membrane potential, firing frequency and action potential characteristics under basal and various test conditions.

\section{Results}

We used Pitx3-GFP mice that express GFP selectively in midbrain dopamine neurons (Zhao et al. 2004), to conduct targeted whole-cell recordings from vlPAG/DRN DA neurons in acute brain slices, an approach that we have previously described (Dougalis et al. 2012). Under these conditions, most
DA neurons in the vlPAG/DRN fire action potentials spontaneously (Dougalis et al. 2012).

\subsection{Hyperpolarization-activated cation current $\left(\mathrm{I}_{\mathbf{H}}\right)$}

Midbrain DA neurons express a hyperpolarization-activated inward current $\left(\mathrm{I}_{\mathrm{H}}\right)$ whose blockade can decrease pacemaker frequency of some, but not all, SNc DA neurons (see Neuhoff et al. 2002; Seutin et al. 2001; Khaliq and Bean 2010). The $\mathrm{I}_{\mathrm{H}}$ current recorded in vlPAG/DRN DA neurons was sensitive to the specific $\mathrm{I}_{\mathrm{H}}$ current blocker ZD7288 $(30 \mu \mathrm{M})$ which completely ablated the inward currents recorded under a hyperpolarizing pulse from -62 to $-132 \mathrm{mV}$ in voltage-clamp $(87 \pm 4 \%$ current block, $n=6$, from three mice, Fig. 1a). A series of hyperpolarizing pulses from $-52 \mathrm{mV}$ to $-152 \mathrm{mV}$ for $1 \mathrm{~s}$ in the presence of TTX ( $n=6$, three mice, Fig. 1b) resulted in $\mathrm{I}_{\mathrm{H}}$ current steadystate activation values for $\mathrm{V}_{50}$ and slope of $-114.7 \pm 1.7 \mathrm{mV}$ and $-12.7 \pm 1.6$ respectively (Fig. 1c). $\mathrm{I}_{\mathrm{H}}$ current activation time constant was voltage-dependent becoming faster at more positive potentials $(2118 \pm 664 \mathrm{~ms}$ at $-102 \mathrm{mV}$ and $151.0 \pm 24 \mathrm{~ms}$ at $-152 \mathrm{mV}, n=6, P<0.001$, paired t-test) with $\mathrm{V}_{50}$ and slope values of $-112.7 \pm 7.8 \mathrm{mV}$ and $6.1 \pm 5.1$ respectively (Fig. 1d). Maximal $\mathrm{I}_{\mathrm{H}}$ conductance was calculated to be $0.56 \pm 0.01 \mathrm{nS}$ at $-152 \mathrm{mV}(n=6)$. Results quantifying $\mathrm{I}_{\mathrm{H}}$ current steady-state activation and activation kinetics via a current subtraction protocol (before and after addition of ZD7288) did not differ from results obtained using the simplified activation voltage-clamp protocol used above and thus the subtraction protocol was not routinely used for measurements of $\mathrm{I}_{\mathrm{H}}$ current kinetics of vlPAG/DRN DA neurons (data not shown). To evaluate the impact of $\mathrm{I}_{\mathrm{H}}$ current blockade on autorhythmicity and pacemaker frequency of DA neurons, ZD7288 $(30 \mu \mathrm{M})$ was perfused under current-clamp conditions while monitoring spontaneous firing in the presence of synaptic blockers for glutamatergic and GABAergic transmission (see methods). The $\mathrm{I}_{\mathrm{H}}$ current blocker, did not affect firing frequency of DA neurons (firing frequency in control, $3.1 \pm 0.5 \mathrm{~Hz}$; in ZD 7288, $3.5 \pm 0.8 \mathrm{~Hz}, n=7$ from four mice, $P>0.05$, paired t-test, Fig. 1e, g) or firing regularity (CV-ISI in control, $0.46 \pm 0.07$; in ZD7228, $0.44 \pm 0.07, n=7$ from four mice, $P>0.05$, paired t-test, Fig. 1h) despite eliminating the $\mathrm{I}_{\mathrm{H}}$ mediated voltage-sag under current-clamp induced by hyperpolarizing current injections ( $-60 \mathrm{pA}$ for $1 \mathrm{~s}$, Fig. 1f). For comparative purposes we also recorded $\mathrm{I}_{\mathrm{H}}$ current in $\mathrm{SNc}$ and VTA DA neurons (three mice). As expected, both midbrain nuclei responded with an inward current upon a series of hyperpolarizing voltage steps (from -62 to $-132 \mathrm{mV}$ in $10 \mathrm{mV}$ increments, online resource 1A) albeit with evidently differential current steady-state activation midpoint (online resource 1B) and kinetics (online resource 1C). SNc neurons had more positive $\mathrm{V}_{50}$ values $(\mathrm{SNc},-92 \pm 2.3 \mathrm{mV}, n=6-7$ for each set, $P<0.01$ to both VTA and vlPAG/DRN, ANOVA with Tukey's post-hoc test) for $\mathrm{I}_{\mathrm{H}}$ current activation and a significantly faster activation time constant (at $-132 \mathrm{mV}$, SNc, 
Table 1 A summary of parameters utilized by the vlPAG/DRN DA model neuron

\begin{tabular}{|c|c|c|c|c|c|c|c|c|c|c|}
\hline \multirow[t]{2}{*}{ Current } & \multirow[t]{2}{*}{$\mathrm{E}_{\mathrm{rev}}(\mathrm{mV})$} & \multirow[t]{2}{*}{$\mathrm{G}_{\max }\left(\mathrm{mS} / \mathrm{cm}^{2}\right)$} & \multicolumn{2}{|c|}{$\begin{array}{l}\text { Activation } \\
\text { function }(\mathrm{A})\end{array}$} & \multicolumn{2}{|c|}{$\begin{array}{l}\text { Inactivation } \\
\text { function (B) }\end{array}$} & \multicolumn{2}{|c|}{$\begin{array}{l}\text { Activation function } \\
\text { time constant }\left(\tau_{\mathrm{A}}\right)\end{array}$} & \multicolumn{2}{|c|}{$\begin{array}{l}\text { Inactivation function } \\
\text { time constant }\left(\tau_{\mathrm{B}}\right)\end{array}$} \\
\hline & & & $\mathrm{V}_{50}(\mathrm{mV})$ & slope & $\mathrm{V}_{50}(\mathrm{mV})$ & slope & $\mathrm{V}_{50}(\mathrm{mV})$ & slope & $\mathrm{V}_{50}(\mathrm{mV})$ & Slope \\
\hline $\mathrm{I}_{\mathrm{A}}$ & -73 & 6 & -57.5 & 7.7 & -93 & -6.1 & -68.8 & -5.0 & -24.6 & -8.6 \\
\hline $\mathrm{I}_{\mathrm{Kdr}}$ & -73 & 3 & -25.0 & 12.0 & NA & NA & -38.4 & -6.9 & NA & NA \\
\hline $\mathrm{I}_{\mathrm{M}}$ & -73 & 1 & -35.0 & 8.5 & NA & NA & -27.9 & -6.9 & NA & NA \\
\hline $\mathrm{I}_{\mathrm{NaT}}$ & +50 & 20 & -44.0 & 4.5 & -62.0 & -6.5 & -28.0 & -7.0 & -14.5 & -9.5 \\
\hline $\mathrm{I}_{\mathrm{NaP}}$ & +50 & 0.02 & -57.0 & 3.5 & NA & NA & $\#$ & $\#$ & NA & NA \\
\hline $\mathrm{I}_{\mathrm{CaHVA}}$ & +120 & 0.04 & -22.0 & 5.0 & -40.0 & -7.0 & -40.0 & -3 & -39.0 & -2.6 \\
\hline $\mathrm{I}_{\mathrm{CLVA}}$ & +120 & 0.04 & -57.5 & 6.5 & $-83.0 *$ & $-6.1 *$ & -68.8 & -5.0 & $-24.6^{*}$ & $-8.6^{*}$ \\
\hline $\mathrm{I}_{\mathrm{H}}$ & -40 & 0.08 & -114.7 & -12.8 & NA & NA & -112.7 & 6.7 & NA & NA \\
\hline$I_{\text {leak }}$ & -55 & 0.04 & NA & & & & & & & \\
\hline
\end{tabular}

The values of reversal potential $\left(\mathrm{E}_{\mathrm{rev}}\right)$ and maximal conductance $\left(\mathrm{G}_{\mathrm{max}}\right)$ for each ionic current in the model DA neuron are presented (columns two and three). Columns four to seven detail the mean half-maximal $\left(V_{50}\right)$ and slope values for the voltage-dependence of the extent of activation/inactivation of the gating functions and voltage-dependence of the rate of activation/inactivation of the gating functions derived by first order Boltzmann function fitting to the electrophysiological data (see methods). All values have been corrected for liquid junction potentials

*, electrophysiological estimation; NA, not applicable; \#, function does not exhibit voltage-dependency in activation rate and is held constant at $0.1 \mathrm{~ms}$

$114 \pm 14 \mathrm{~ms}, n=6-7$ for each set, $P<0.05$ to both VTA and vlPAG/DRN, ANOVA with Tukey's post-hoc test) than either VTA $(-121 \pm 5.6 \mathrm{mV}$ and $397 \pm 84 \mathrm{~ms}$ respectively) or vlPAG/ DRN $(-121 \pm 3.5 \mathrm{mV}$ and $260 \pm 34 \mathrm{~ms}$ respectively) that exhibited comparable profiles without any significant differences between them in either parameter (VTA $v s$ vlPAG/ DRN, $P>0.05$ for both comparisons, unpaired t-test). Also no differences were detected in the slope of the activation curve (slope, SNc, $-11.8 \pm 2.6$; VTA, $-12.0 \pm 2.8$; vlPAG/DRN, $-13.0 \pm 1.8, n=6-7$ for each set, $P>0.05$, ANOVA with Tukey's post-hoc test).

\subsection{A-type $\left(I_{A}\right)$ transient potassium currents}

One of the hallmarks of the electrophysiological behavior of DA neurons in the vlPAG/DRN brain slice is the pronounced outward tail current recorded at subthreshold potential ( -60 to $-50 \mathrm{mV}$ ) following a hyperpolarizing pulse (beyond $-85 \mathrm{mV}$ ) under voltage-clamp (Fig. 2a). The amplitude of the outward transient tail conductance after a hyperpolarizing step was suppressed $(90.1 \pm 2.4 \%$ at $-62 \mathrm{mV}, n=6$ from three mice) by $2-$ $3 \mathrm{mM}$ 4-aminopyridine (4-AP) indicating that it represents an A-type transient outward potassium conductance $\left(\mathrm{I}_{\mathrm{A}}\right.$ current, Fig. 2a, b). Subtraction of the 4-AP sensitive current lead to the typical 'current crossing' (Fig. 2c) as reported previously by others (Khaliq and Bean 2008) suggesting that 4-AP also increases some background conductance while blocking the $\mathrm{I}_{\mathrm{A}}$ current (and potentially blocking a fast delayed rectifier conductance in some neurons, see Itri et al. 2005) making its kinetic description difficult. An alternative method to examine $\mathrm{I}_{\mathrm{A}}$ current steady-state activation is using digital current subtraction between two different activation protocols in the presence of TTX $(1 \mu \mathrm{M})$ as reported previously (Koyama and Appel 2006a). Responses to protocol one (Fig. 2d) activated transient and sustained outward currents while responses to protocol two activated slowly-activating sustained outward currents only (reminiscent of delayed rectifier potassium currents, Fig. 2d). Subtraction of the responses (protocol 1 minus protocol 2) resulted in isolation of the $\mathrm{I}_{\mathrm{A}}$ current (Fig. 2e). Steady-state inactivation was studied using a single standard inactivation protocol without digital current subtraction since the peak of the $\mathrm{I}_{\mathrm{A}}$ current at the test voltage studied $(-47 \mathrm{mV})$ was not contaminated by slowly activating outward currents (Fig. 2f). Maximal $\mathrm{I}_{\mathrm{A}}$ conductance was calculated to be $10.0 \pm 1.1 \mathrm{nS}$ at $+8 \mathrm{mV}$ ( $n=6$, from four mice). The $\mathrm{V}_{50}$ value for steady-state activation was $-57.5 \pm 0.5 \mathrm{mV}$ with a slope of $7.7 \pm 0.4$, while the corresponding values of $\mathrm{V}_{50}$ for steady-state inactivation and its slope were $-87.4 \pm 0.8 \mathrm{mV}$ and $-6.1 \pm 0.8$ respectively $(n=6$, Fig. 2g). Activation time constant for $\mathrm{I}_{\mathrm{A}}$ current was voltagedependent becoming faster at more positive potentials $(5.45 \pm 0.27 \mathrm{~ms}$ at $-62 \mathrm{mV}$ and $0.95 \pm 0.06 \mathrm{~ms}$ at $+8 \mathrm{mV}$, $n=6, P<0.001$, paired t-test) with a $\mathrm{V}_{50}$ and slope values of $-68.8 \pm 0.7 \mathrm{mV}$ and $-5.0 \pm 0.7$ respectively (Fig. 2h). Inactivation kinetics were also voltage-dependent with faster inactivation at more positive potentials $(128.3 \pm 9.5 \mathrm{~ms}$ at $-62 \mathrm{mV}$ and $50.8 \pm 10.0 \mathrm{~ms}$ at $+8 \mathrm{mV}, n=6, P<0.01$, paired t-test) with $\mathrm{V}_{50}$ and slope values of $-24.6 \pm 5.7 \mathrm{mV}$ and $-8.6 \pm 5.3$ respectively (Fig. 2i). A second, voltage-independent, slow inactivation time constant $\left(\tau_{\text {slow }}\right)$ could be fitted to $I_{A}$ currents recorded more positively than $-30 \mathrm{mV}$ and contributed to approximately $35 \%$ and $50 \%$ of the maximal $\mathrm{I}_{\mathrm{A}}$ current amplitude recorded at -22 and $+8 \mathrm{mV}$ respectively $(357 \pm 53 \mathrm{~ms}$ at $-22 \mathrm{mV}$ and $562 \pm 106 \mathrm{~ms}$ at $+8 \mathrm{mV}, n=6$, $P>0.05$, paired t-test, online resource $2 \mathrm{~A}$ ). We did not further 

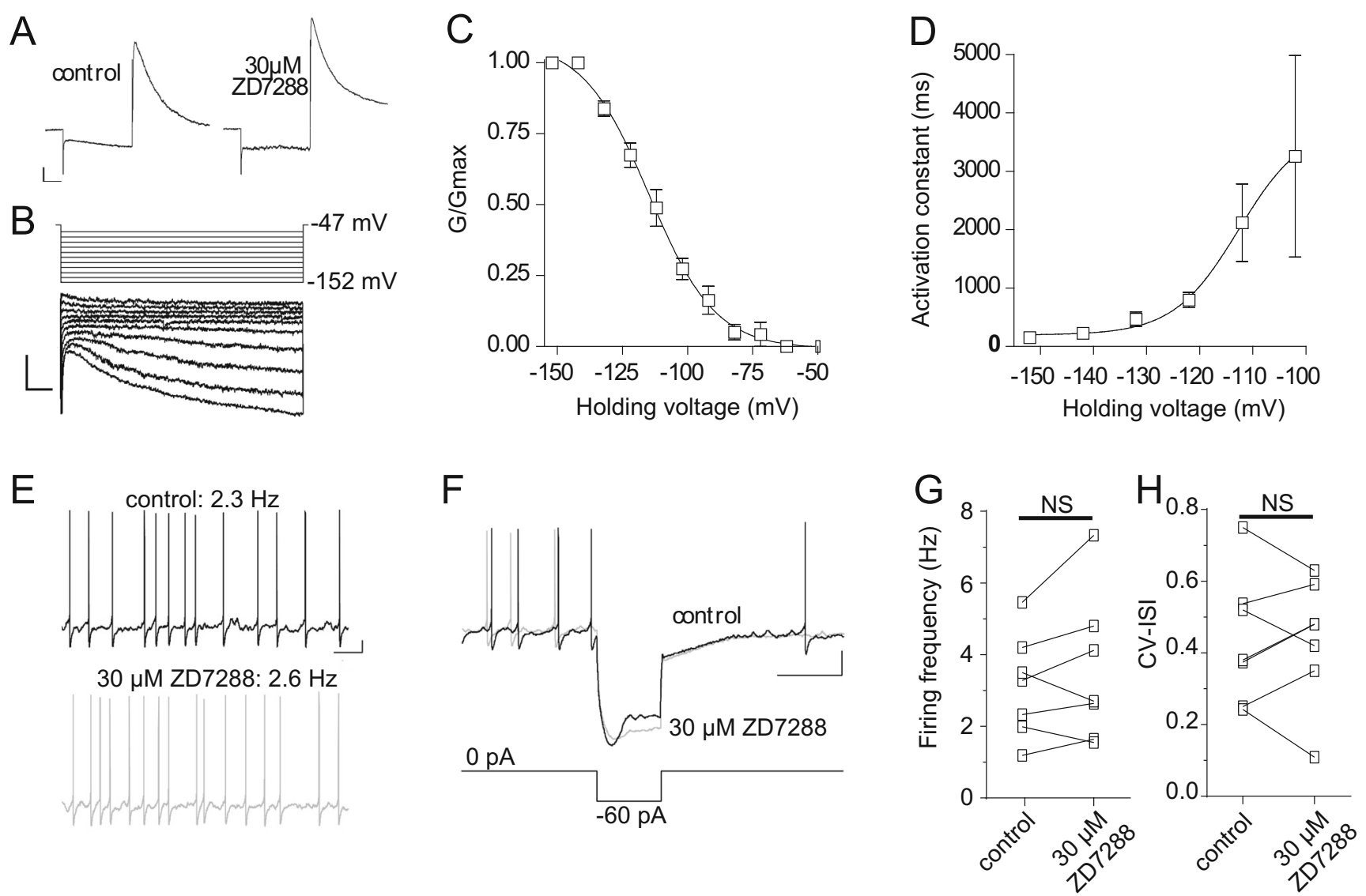

Fig. 1 Hyperpolarization-activated cation current ( $\mathrm{I}_{\mathrm{H}}$ current). a. Representative voltage-clamp electrophysiological traces from a vlPAG/ DRN DA neuron showing the expression of a hyperpolarization-activated inward current $\left(\mathrm{I}_{\mathrm{H}}\right)$ through a single hyperpolarizing step from -62 to $-152 \mathrm{mV}$ (500 ms duration). This current was sensitive to ZD 7288 $(30 \mu \mathrm{M})$, a reputed specific blocker of the $\mathrm{I}_{\mathrm{H}}$ conductance (scale bars, 50 pA and $125 \mathrm{~ms}$ ). b. Electrophysiological traces of $\mathrm{I}_{\mathrm{H}}$ current activation taken from a vlPAG/DRN DA neuron. A series of voltage steps in $10 \mathrm{mV}$ increments was delivered (holding potential $-47 \mathrm{mV}$ ) from -62 to $-152 \mathrm{mV}$ for $1 \mathrm{~s}$ (scale bars, $25 \mathrm{pA}$ and $100 \mathrm{~ms}$ ). c. Average steady-state activation curve $\left(\mathrm{G} / \mathrm{G}_{\max }\right)$ for the $\mathrm{I}_{\mathrm{H}}$ conductance for vlPAG/DRN neurons recorded through a series of hyperpolarizing voltage steps from $-52 \mathrm{mV}$ to -152 in $10 \mathrm{mV}$ increments as shown in $\mathrm{B}(n=6)$. Normalised conductance plots were fitted with a single Boltzmann function to calculate mean $V_{50}$ and slope values (activation, $-114 \mathrm{mV}$; slope, $-12.7 ; n=6$ ). d. Voltagedependence of $\mathrm{I}_{\mathrm{H}}$ current activation time constant. Activation time constant was voltage-dependent and become faster at more positive potentials (mean $\tau_{\text {act }}$ of $151 \mathrm{~ms}$ at $-152 \mathrm{mV}$ ). Data were plotted against holding voltage and fitted with a single Boltzmann function to calculate

investigate the origin of this slow conductance but we noted interestingly that 4-AP subtracted currents (as shown in Fig. 2c) not only exhibited a similar average activation steady-state curve $\left(\mathrm{V}_{50}\right.$ of $-61.4 \pm 0.9$ and slope of $7.1 \pm 0.8, n=3$, online resource $2 \mathrm{~B}$ ) to currents recorded here with the two protocol current subtraction method, but more importantly, 4-AP sensitive currents also exhibited consistently a bi-exponential decay with commensurate, voltage-dependent, fast and slow inactivation time constants that differentially contributed to current amplitude at a range of holding voltages (online resource 2C, D). mean $\mathrm{V}_{50}(-112.7 \mathrm{mV})$ and slope $(6.1)$ values $(n=6)$. e. Representative current-clamp electrophysiological traces taken from a vlPAG/ DRN DA neuron showing the effect of ZD $7288(30 \mu \mathrm{M})$ on firing frequency. The $\mathrm{I}_{\mathrm{H}}$ blocker did not have any effects on the frequency of firing on this cell (control, $2.3 \mathrm{~Hz}$ and in ZD 7288, $2.6 \mathrm{~Hz}$ ) (scale bars, $10 \mathrm{mV}$ and $1 \mathrm{~s}$ ). f. Representative current-clamp electrophysiological traces from the neuron in E showing the effect of ZD $7288(30 \mu \mathrm{M})$ on the hyperpolarizationinduced voltage-sag. The $\mathrm{I}_{\mathrm{H}}$ blocker blocked the voltage-sag in response to hyperpolarizing current injection ( $-60 \mathrm{pA}$, scale bars, $20 \mathrm{mV}$ and $1 \mathrm{~s})$. g. Bar chart comparison of average firing frequency $(\mathrm{Hz})$ before and after application of ZD $7288(30 \mu \mathrm{M})$ as shown in E for eight vlPAG/DRN DA neurons. ZD 7288 did not induce any significant change in the firing frequency of vlPAG/DRN DA neurons (mean firing frequency in control, $3.1 \mathrm{~Hz}$; in ZD 7288, $3.5 \mathrm{~Hz}, n=7$, paired t-test; NS, not significant). H. Bar chart comparison of average CV-ISI before and after application of ZD $7288(30 \mu \mathrm{M})$ as shown in E for seven DA neurons. ZD 7288 did not induce any significant change in CV-ISI of vlPAG/DRN DA neurons (mean CV-ISI in control, 0.46; in ZD 7288, 0.44, $n=7$, paired t-test; NS, not significant)

To examine further the properties of $\mathrm{I}_{\mathrm{A}}$ currents and to compare our results obtained with the methods described above we recruited $\mathrm{I}_{\mathrm{A}}$ currents via a single activation protocol (protocol 1) in the presence of $10 \mathrm{mM}$ TEA (as described previously by Silva et al. 1990) a method that does not rely on digital current subtraction. This resulted in recording $\mathrm{I}_{\mathrm{A}}$ currents (Fig. 2j) with analogous average activation steadystate $\mathrm{V}_{50}$ of $-55.6 \pm 1.0 \mathrm{mV}$, but shallower activation slope of $15.1 \pm 1.1(n=5$, from three mice, online resource $2 \mathrm{E})$, to the two protocol subtraction method and the 4-AP-sensitive $\mathrm{I}_{\mathrm{A}}$ 


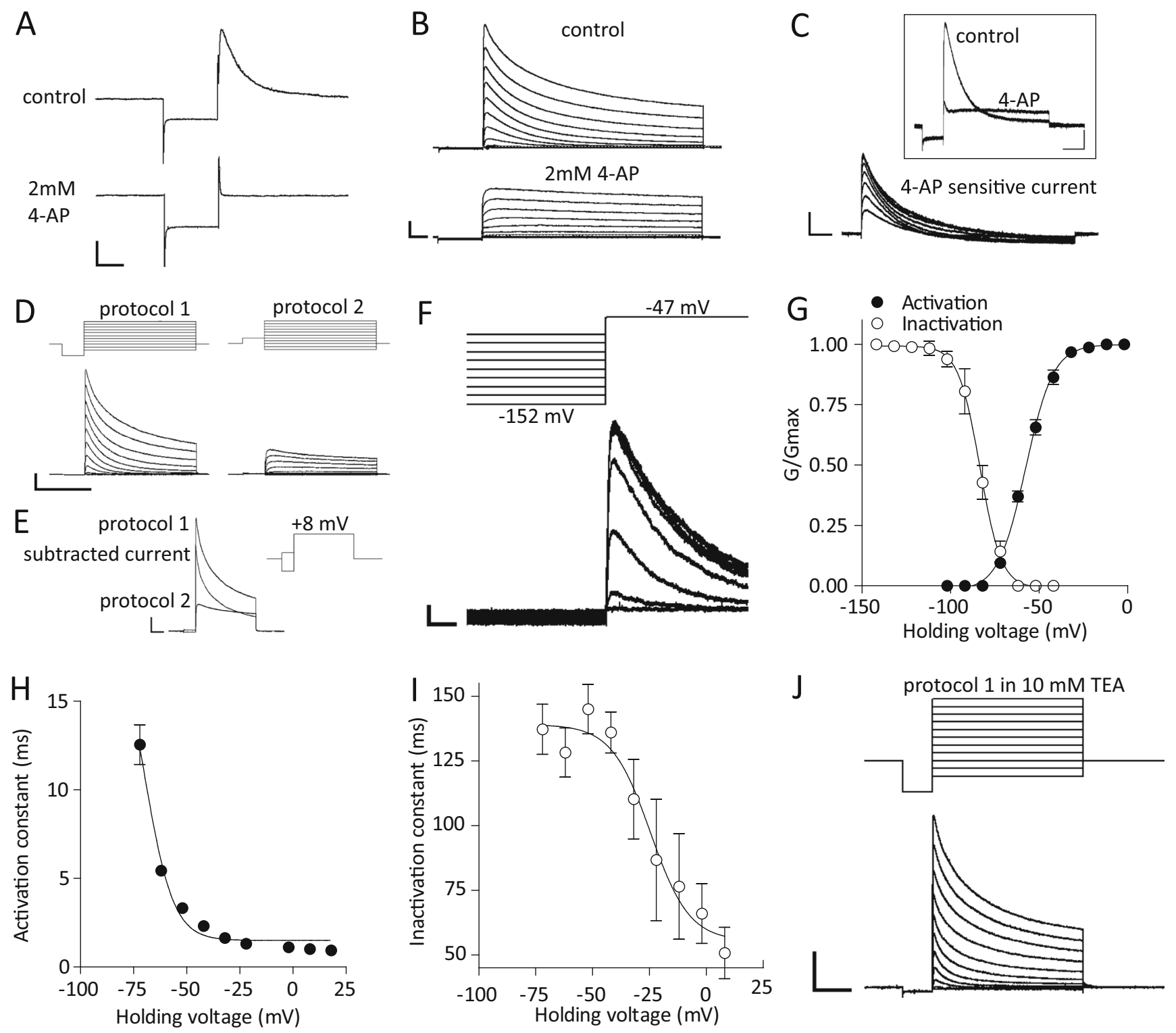

currents. Interestingly, the single protocol $\mathrm{I}_{\mathrm{A}}$ current isolation in TEA also resulted in a bi-exponential inactivation of recorded $\mathrm{I}_{\mathrm{A}}$ currents with differential contributions to current amplitude at different voltages (online resource 2F, G). However, unlike the previous methods, inactivation kinetics under these circumstances did not exhibit strong voltage dependence at positive potentials (online resource $2 \mathrm{~F}$ ). Both 4-AP-sensitive $\mathrm{I}_{\mathrm{A}}$ currents and $\mathrm{I}_{\mathrm{A}}$ currents isolated in TEA exhibited commensurate voltage-dependent activation kinetics (faster at more positive potentials) in good agreement with the two protocol current subtraction method (data not shown).

\subsection{Delayed rectifier $\left(\mathrm{I}_{\mathrm{Kdr}}\right)$ sustained potassium currents}

Sustained, non-inactivating/slowly inactivating, outward currents reminiscent of the delayed rectifier $\mathrm{I}_{\mathrm{Kdr}}$ were isolated using KGlu based-internal solution in the presence of TTX $(1 \mu \mathrm{M})$ via a standard voltage-clamp activation protocol as reported previously (Silva et al. 1990). Currents recruited were slowly activating, largely non-inactivating during the depolarizing $1 \mathrm{~s}$ step, exhibiting a fast deactivation upon return to negative holding potentials and sensitivity to tetraethylammonium (TEA, $10 \mathrm{mM}$ ) suggesting the presence of a delayed rectifier current $\mathrm{I}_{\mathrm{Kdr}}$ in vlPAG/DRN DA neurons (Fig. 3a). Maximal $\mathrm{I}_{\mathrm{Kdr}}$ conductance was calculated to be $7.0 \pm 0.6 \mathrm{nS}$ at $+8 \mathrm{mV}(n=6$, from four mice). The $\mathrm{V}_{50}$ value for steady-state activation was $-26.9 \pm 1.7 \mathrm{mV}$ with a slope of $13.4 \pm 1.8(n=6$, Fig. $3 \mathrm{~b})$. Activation time constant for $\mathrm{I}_{\mathrm{Kdr}}$ current was voltage-dependent becoming faster at more positive potentials $(98.1 \pm 7.7 \mathrm{~ms}$ at $-52 \mathrm{mV}$ and $2.8 \pm 0.2 \mathrm{~ms}$ at $+8 \mathrm{mV}, n=6, P<0.001$, paired $\mathrm{t}-$ test) with $\mathrm{V}_{50}$ and slope of values of $-38.4 \pm 1.0 \mathrm{mV}$ and $-6.8 \pm 0.9$ respectively (Fig. $3 \mathrm{c}$ ). The $\mathrm{I}_{\mathrm{Kdr}}$ current did not exhibit any appreciable inactivation even on long $10 \mathrm{~s}$ pulses at potentials up to $-20 \mathrm{mV}$. We also monitored the deactivation of the 
4 Fig. 2 A-type $\left(\mathrm{I}_{\mathrm{A}}\right)$ potassium current. a. Electrophysiological traces of voltage-clamp recordings (single step from -62 to $-102 \mathrm{mV}$ for $250 \mathrm{~ms}$ ) from a vlPAG/DRN DA neuron before and after addition of 4aminopyridine (4-AP, $2 \mathrm{mM}$ ) in the presence of TTX $(1 \mu \mathrm{M})$. The outward tail current elicited after the end of the hyperpolarizing step (return to the holding potential of $-62 \mathrm{mV}$ ) was completely blocked by the $\mathrm{I}_{\mathrm{A}}$ potassium current blocker 4-AP (scale bars, $50 \mathrm{pA}$ and $100 \mathrm{~ms}$ ). b. Electrophysiological traces of voltage-clamp recordings (prepulse step of $250 \mathrm{~ms}$ from -62 to $-112 \mathrm{mV}$ before a series of test steps of $1 \mathrm{~s}$ in duration from -92 to $+8 \mathrm{mV}$ in $10 \mathrm{mV}$ increments) from a vlPAG/DRN DA neuron before and after addition of 4-aminopyridine (4-AP, $2 \mathrm{mM}$ ). The transient outward currents elicited upon depolarization were blocked by the $\mathrm{I}_{\mathrm{A}}$ potassium current blocker 4-AP leaving a residual sustained current (scale bars, $500 \mathrm{pA}$ and $100 \mathrm{~ms}$ ). c. Overlaid electrophysiological traces from experiment shown in B depicting transient outward currents (test pulse to $-52 \mathrm{mV}$ ) before and after addition of 4-aminopyridine (4-AP, $2 \mathrm{mM}$, inset, scale bars $100 \mathrm{pA}$ and $200 \mathrm{~ms}$ ) accompanied by an overlay of the digitally subtracted 4-AP sensitive current (bottom traces, scale bars $200 \mathrm{pA}$ and $200 \mathrm{~ms}$ ) for the whole series of steps shown in B (depicted test steps from -72 to $-12 \mathrm{mV}$ ). Note that the currents before and after 4-AP 'cross' signifying that 4-AP blocks the $\mathrm{I}_{\mathrm{A}}$ transient outward current but also increases a background conductance. $\mathbf{d}$. Activation of transient and sustained outward potassium currents recorded using a series of depolarizing test pulses ( $1 \mathrm{~s}$ duration, from -92 to $+8 \mathrm{mV}, 10 \mathrm{mV}$ increments) via two different single prepulses ( $250 \mathrm{~ms}$ duration from -72 to $-112 \mathrm{mV}$ and -72 to $-52 \mathrm{mV}$, protocols 1 and 2 respectively) in the presence of TTX $(1 \mu \mathrm{M})$. Protocol 1 recruited fast activating transient and sustained outward currents using a prepulse to $-112 \mathrm{mV}$ to facilitate $\mathrm{I}_{\mathrm{A}}$ current's recovery from inactivation, while protocol 2 recruited only slowly activating sustained outward currents using a prepulse to $-52 \mathrm{mV}$ to inactivate $\mathrm{I}_{\mathrm{A}}$ current (scale bars, $500 \mathrm{pA}$ and $500 \mathrm{~ms}$ ). Digital subtraction of the responses (protocol 1 minus protocol 2) was used to isolate the transient $\mathrm{I}_{\mathrm{A}}$ potassium conductance from background sustained currents. e. Overlaid electrophysiological traces obtained via voltage protocols 1 and

$\mathrm{I}_{\mathrm{Kdr}}$ tail current upon return to a single potential of $-72 \mathrm{mV}$ following the series of test potentials used to recruit the $\mathrm{I}_{\mathrm{Kdr}}$ current as described above (Fig. 2d). Deactivation of the $\mathrm{I}_{\mathrm{Kdr}}$ tail currents at $-72 \mathrm{mV}$ was sensitive to the test voltage potential prior to return to $-72 \mathrm{mV}$ and became faster at more positive test holding voltages $(32.3 \pm 2.0 \mathrm{~ms}$ following return from $-52 \mathrm{mV}$ and $17.2 \pm 1.7 \mathrm{~ms}$ following return from $+8 \mathrm{mV}, n=6, P<0.001$, paired t-test) with $\mathrm{V}_{50}$ and slope of values of $-38.9 \pm 12 \mathrm{mV}$ and $-11.5 \pm 6.7$ respectively (Fig. $3 \mathrm{e}$ ). Activation of delayed rectifier-type currents is usually associated with Kv2.1 and/or Kv2.2 families of voltage-gated potassium channels in sympathetic neurons (Malin and Nerbonne 2002) and SNc DA dissociated neurons (Kimm et al. 2015). $\mathrm{A} \mathrm{K}_{\mathrm{V}} 7$ (KCNQ)-mediated current (also known as the M-type current, $\mathrm{I}_{\mathrm{M}}$ ) has been has been described in VTA neurons (Koyama and Appel 2006b). The $\mathrm{I}_{\mathrm{M}}$ potassium current is a slowly activating, non-inactivating current with sensitivity to TEA (through Kv7.2 subunits, Hadley et al. 2000) and activation that partially overlaps with that of delayed rectifier currents (Brown and Adams 1980; see also review, Brown and Passmore 2009). To examine the possibility of the presence of an $\mathrm{I}_{\mathrm{M}}$ current in vlPAG/DRN DA neurons we used a modified deactivation tail protocol (Koyama and Appel 2006b) where neurons were held at $-72 \mathrm{mV}$ and given an $1 \mathrm{~s}$ prepulse
2 (taken from D). Responses for each protocol together with the resultant digitally subtracted $\mathrm{I}_{\mathrm{A}}$ current (protocol 1 minus protocol 2) are shown at a single test voltage step of $+8 \mathrm{mV}$ for clarity (scale bars, $500 \mathrm{pA}$ and $200 \mathrm{~ms}$ ). f. Inactivation of transient $\mathrm{I}_{\mathrm{A}}$ outward potassium current recorded using a series of depolarizing prepulses ( $1 \mathrm{~s}$ duration, from -152 to $-52 \mathrm{mV}$ ) to differentially recover $\mathrm{I}_{\mathrm{A}}$ current followed by a test pulse (to $-47 \mathrm{mV}$ ) to record its activation as a function of the prepulse potential (scale bars, $100 \mathrm{pA}$ and $50 \mathrm{~ms}$ ). g. Average steady-state activation and inactivation curves $\left(\mathrm{G} / \mathrm{G}_{\max }\right.$ against holding voltage) for the subtracted (protocol 1 minus protocol 2) $\mathrm{I}_{\mathrm{A}}$ current. Normalised conductance plots were fitted with a single Boltzmann function to calculate mean $\mathrm{V}_{50}$ (activation,-57.5 mV; inactivation, $-87.4 \mathrm{mV}$ ) and slope (activation, 7.8; inactivation, -6.1$)$ values $(n=6)$. $\mathbf{h}$. Voltage-dependence of $\mathrm{I}_{\mathrm{A}}$ current activation time constant. Subtracted $\mathrm{I}_{\mathrm{A}}$ currents were fitted with a single exponential function (start to peak) at each test holding voltage to calculate the activation time constant. Activation time constant appeared voltagedependent and became faster at more positive potentials (mean $\tau_{\text {act }}$ of $0.95 \mathrm{~ms}$ at $+8 \mathrm{mV}$ ). Data were plotted against holding voltage and fitted with a single Boltzmann function to calculate mean $\mathrm{V}_{50}(-68.8 \mathrm{mV})$ and slope $(-5.0)$ values $(n=6)$. i. Voltage-dependence of $\mathrm{I}_{\mathrm{A}}$ current inactivation time constant. Subtracted $\mathrm{I}_{\mathrm{A}}$ currents were fitted with a single exponential function (peak to end) at each test holding voltage to calculate the inactivation time constant. Inactivation time constant appeared voltagedependent and became faster at more positive potentials (mean $\tau_{\text {ina }}$ of $50.8 \mathrm{~ms}$ at $+8 \mathrm{mV}$ ). Data were plotted against holding voltage and fitted with a single Boltzmann function to calculate mean $\mathrm{V}_{50}(-24.6 \mathrm{mV})$ and slope $(-8.6)$ values $(n=6)$. j. Typical electrophysiological traces obtained via activation voltage protocol 1 (as shown in D) in the presence of $10 \mathrm{mM}$ TEA to block delayed rectifier currents as an alternative method to obtain $\mathrm{I}_{\mathrm{A}}$ currents without digital subtraction between two protocols (e.g. Silva et al. 1990). $\mathrm{I}_{\mathrm{A}}$ currents obtained exhibited analogous behaviour to that obtained via the two protocol subtraction and the 4-AP subtraction methods (see also online resource 2; scale bars, $500 \mathrm{pA}$ and $250 \mathrm{~ms}$ )

to $-32 \mathrm{mV}$ to activate the $\mathrm{I}_{\mathrm{M}}$ current before stepped to -42 to $-72 \mathrm{mV} 1 \mathrm{~s}$ to record the resultant $\mathrm{I}_{\mathrm{M}}$ current deactivation tail in the presence of TTX $(1 \mu \mathrm{M})$ (online resource $2 \mathrm{~A}, \mathrm{~B})$. We recorded $\mathrm{I}_{\mathrm{M}}$ current relaxations (online resource $3 \mathrm{C}$ ) with similar properties to that reported previously for VTA neurons (Koyama and Appel 2006b). These were sensitive to the KCNQ blocker XE991 $(30 \mu \mathrm{M}$, online resource 3A, B, D). Moreover, XE991 partially inhibited the depolarizationinduced sustained outward currents at $-32 \mathrm{mV}(67 \pm 7 \%$ of control, $n=4$, online resource 3D) suggesting that depolarizing pulses recruit effectively a large component of sustained outward current that is carried forward by the KCNQ family of voltage-gated potassium channels. Finally, perfusion with XE991 $(30 \mu \mathrm{M})$ induced a depolarizing current in voltage clamp at $-70 \mathrm{mV}$ (control holding current at $-70 \mathrm{mV}$, $-18.2 \pm 2.1 \mathrm{pA}$, in XE991, $27.2 \pm 1.5 \mathrm{pA}, n=4, P<0.05$, paired t-test, data not shown).

\subsection{Low and high-voltage activated (LVA and HVA) calcium currents $\left(\mathrm{I}_{\mathrm{CaLVA}}\right.$ and $\left.\mathrm{I}_{\mathrm{CaHVA}}\right)$}

Calcium currents play an important role in neuronal autorhythmicity and pacemaking in DA midbrain neurons (Wolfart and Roeper 2002; Puopolo et al. 2007; Khaliq and 


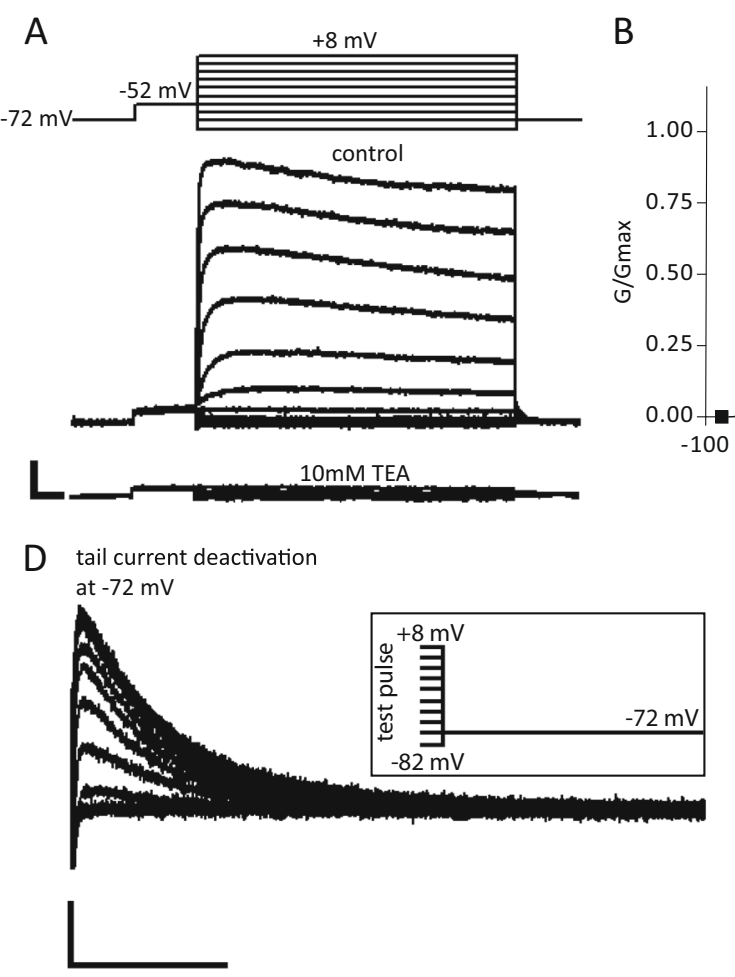

Fig. 3 Delayed rectifier $\left(I_{K d r}\right)$ potassium current. a. Top: Representative electrophysiological traces of slowly-activating, sustained, outward potassium currents $\left(\mathrm{I}_{\mathrm{Kdr}}\right)$ recorded using a prepulse (from- $72 \mathrm{mV}$ to $-52 \mathrm{mV}$ for $250 \mathrm{~ms}$ ) before the delivery of series of depolarizing test steps ( $1 \mathrm{~s}$ duration, from -72 to $+8 \mathrm{mV}, 10 \mathrm{mV}$ increments) in the presence of $1 \mu \mathrm{M}$ TTX (scale bars, $100 \mathrm{pA}, 100 \mathrm{~ms}$ ). Bottom: Sustained outward currents and tail currents were sensitive to TEA $(10 \mathrm{mM})$. b. Average steady-state activation curve $\left(\mathrm{G} / \mathrm{G}_{\max }\right)$ for the slowly-activating, sustained potassium current. Normalised conductance plots were fitted with a single Boltzmann function to calculate mean $\mathrm{V}_{50}(-26.9 \mathrm{mV})$ and slope (13.4) values $(n=6)$. c. Voltage-dependence of $\mathrm{I}_{\mathrm{Kdr}}$ current activation time constant. Currents were fitted with a single exponential function (start to peak) at each test holding voltage to calculate the activation time constant. Activation time constant appeared voltage-dependent and became faster at more positive potentials (mean $\tau_{\text {act }}$ of $2.8 \mathrm{~ms}$ at $+8 \mathrm{mV}$ ). Data were plotted against holding voltage and fitted with a single Boltzmann function to

Bean 2010). We have isolated and recorded calcium currents on vlPAG/DRN DA neurons using standard activation protocols in the presence of blockers of potassium and sodium conductances (Brevi et al. 2001; Pignatelli et al. 2005). Calcium currents exhibited a characteristic inward current-voltage relationship with the maximal amplitude $\left(\mathrm{I}_{\max }\right)$ occurring around -15 to $-10 \mathrm{mV}$ (Fig. 4a) and were fully sensitive to cadmium ( $200 \mu \mathrm{M}, n=4$, from three mice, data not shown). They exhibited a fast, voltage-dependent, activation time constant $\left(\tau_{\text {act }}\right.$, $1.27 \pm 0.28 \mathrm{~ms}$ at $-47 \mathrm{mV}$ and $0.62 \pm 0.06 \mathrm{~ms}$ at $-17 \mathrm{mV}$, $n=4, P<0.05$, paired t-test, Fig. 4b) and inactivated in a voltage-dependent manner during the $250 \mathrm{~ms}$ long steps used (inactivation time constant $\tau_{\text {ina }}, 114 \pm 28 \mathrm{~ms}$ at $-47 \mathrm{mV}$ and $16.7 \pm 1.7 \mathrm{~ms}$ at $-17 \mathrm{mV}, n=4, P<0.05$, paired t-test, Fig. $4 \mathrm{~b}$ ). The corresponding mean $\mathrm{V}_{50}$ values for the voltage dependency of the activation and inactivation time constants were
C
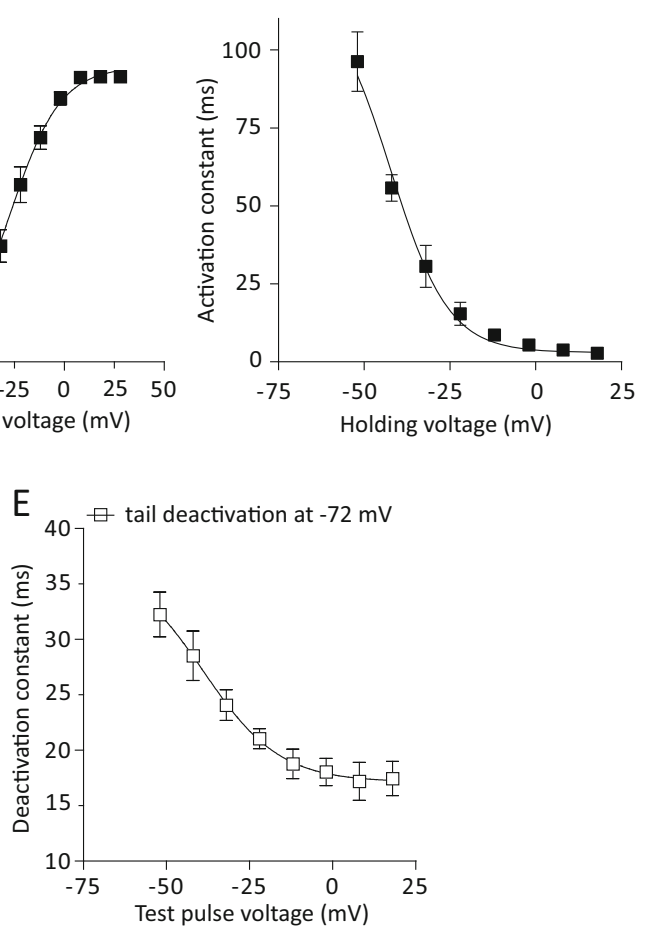

calculate mean $\mathrm{V}_{50}(-38.4 \mathrm{mV})$ and slope $(-6.9)$ values $(n=6)$. d. Overlaid electrophysiological traces of deactivating tail currents recorded at $-72 \mathrm{mV}$. Tail currents were evaluated at $-72 \mathrm{mV}$ following a prepulse (to $-52 \mathrm{mV}$ ) and a series of depolarizing test voltage steps (protocol as in A, shown truncated in the inset for clarity) that recruited the $\mathrm{I}_{\mathrm{kdr}}$ current (scale bars, $50 \mathrm{pA}, 25 \mathrm{~ms}$ ). e. $\mathrm{I}_{\mathrm{Kdr}}$ tail current deactivation time constant recorded at $-72 \mathrm{mV}$ as a function of a series of depolarizing test holding voltages. Tail currents were fitted with a single exponential function (peak to end) and results are plotted against test holding voltage before returning to $-72 \mathrm{mV}$. Deactivation time constant appeared dependent on test voltage and became faster at $-72 \mathrm{mV}$ when neurons were returned to that potential from more positive test potentials (mean $\tau_{\text {deact }}$ of $32 \mathrm{~ms}$ following a test pulse at $-52 \mathrm{mV}$ and $17 \mathrm{~ms}$ following a test pulse at $+8 \mathrm{mV}$ ). Data were plotted against test holding voltage and fitted with a single Boltzmann function to calculate mean $\mathrm{V}_{50}(-38.9 \mathrm{mV})$ and slope $(-11.5)$ values $(n=6)$

$-23.9 \pm 4.3 \mathrm{mV}$ and $-39.0 \pm 2.1 \mathrm{mV}$ with a slope of $-6.9 \pm 3.8$ and $-2.6 \pm 2.1$ respectively $(n=4)$. Mean steadystate activation $\mathrm{V}_{50}$ for the recorded calcium currents using a series of voltage steps was $-26.1 \pm 0.7 \mathrm{mV}$ with a slope value of $5.0 \pm 0.6(n=4$, Fig. $4 \mathrm{c})$. Using fast voltage-ramps $(500 \mathrm{mV} /$ $\mathrm{s}$, from $-107 \mathrm{mV}$ to $+53 \mathrm{mV}$ ) as an alternative way of describing background calcium currents we found that we could successfully discriminate in a subset of vlPAG/DRN DA neurons ( $n=3$ of 7 neurons tested) two peaks of different amplitude (fully sensitive to cadmium), a small one peaking at around -60 to $-50 \mathrm{mV}$ and a larger one peaking at around -20 to $-10 \mathrm{mV}$ (Fig. 4d, e). Analysis of the voltage ramps suggested that LVA calcium currents had a mean steady-state activation $\mathrm{V}_{50}$ of $-61.2 \pm 2.1 \mathrm{mV}$ with a slope value of $3.9 \pm 0.9(n=3)$ while HVA calcium currents exhibited a mean steady-state activation $\mathrm{V}_{50}$ of $-22.4 \pm 1.7 \mathrm{mV}$ with a slope value of $7.1 \pm 1.0(n=3)$. 
To test the effects of calcium current abolition on spontaneous firing we replaced calcium ions with magnesium and recorded in current-clamp mode using KGlu-filled electrodes in standard $\mathrm{aCSF}$ in the presence of synaptic blockers $(n=6$ from three mice, Fig. 4f). We found that this replacement did not prevent expression of spontaneous firing and that it significantly accelerated pacemaking frequency (control firing frequency, $3.7 \pm 0.6$; firing frequency in zero calcium, $5.6 \pm 0.8, n=6$, $P<0.01$, paired t-test, Fig. $4 \mathrm{~g}$ ) without affecting firing regularity (mean CV-ISI and median in $2 \mathrm{mM}$ calcium, $0.66 \pm 0.29$ and 0.42 ; mean CV-ISI and median in zero mM calcium, $0.44 \pm 0.06$ and $0.35, n=6, P>0.05$, paired t-test, Fig. 4h) suggesting that calcium (and calcium-dependent) currents modulate basal firing frequency but are not responsible in driving spontaneous firing of vlPAG/DRN DA neurons.

\subsection{Low and high-voltage activated (LVA and HVA) barium currents ( $\mathrm{I}_{\mathrm{BaLVA}}$ and $\left.\mathrm{I}_{\mathrm{BaHVA}}\right)$}

Calcium currents are known to undergo two types of inactivation (voltage-dependent and calcium-dependent) in a number of preparations (Giannattasio et al. 1991; Haack and Rosenberg 1994; Catterall 2000; Budde et al. 2002). It is unlikely that the inactivation observed here in calcium during the series of depolarizing voltage steps (starting at $-67 \mathrm{mV}$ ) is due to a transient low voltage activated (LVA) calcium current as this voltage protocol should not have recruited such currents to any significant degree while the inactivation kinetics of LVA transient currents are reported to be different from the inactivation seen here for calcium currents (Kang and Kitai 1993a; Pignatelli et al. 2005, see also below the inactivation of barium LVA currents). The inactivation of calcium currents recorded here during long steps could be explained by intracellular calcium release inducing calcium current inactivation or could be attributed to the specific properties of a high voltage activated (HVA) calcium conductance (e.g. see Keja et al. 1992; Kang and Kitai 1993a, 1993b). To distinguish between these possibilities, we replaced calcium $(2 \mathrm{mM})$ with barium $(1 \mathrm{mM})$ in the extracellular solution to reduce calcium release-induced calcium current inactivation (Hille 2001). We used two protocols to record barium currents, commencing from a different potential that can recruit selectively HVA or a composite of LVA/HVA currents (see Brevi et al. 2001; Hille 2001). Barium replacement resulted in robust non-inactivating HVA currents during long steps (250 ms) used in our study (Fig. 5a) suggesting that calcium release-induced calcium current inactivation is the most likely explanation for the observed inactivation of calcium currents (compare Figs. 4a and 5a). During LVA/HVA composite current protocol recording we noticed the appearance of transient fast activating, fast inactivating barium current (Fig. 5a). These currents were subtracted from HVA currents to reveal the LVA component (Fig. 5b) and both components were individually subjected to kinetic analysis. Maximal conductance $\left(\mathrm{G}_{\max }\right)$ for the LVA and HVA calcium component was calculated at $0.65 \pm 0.14 \mathrm{nS}$ and $1.06 \pm 0.18 \mathrm{nS}$ respectively $(n=6$, from three mice). Mean steady-state activation $\mathrm{V}_{50}$ for LVA and HVA barium currents were $-58.9 \pm 1.5 \mathrm{mV}$ and $-34.6 \pm 0.9 \mathrm{mV}$ with slope values of $6.3 \pm 1.2$ and $5.1 \pm 0.8$ respectively $(n=6$, Fig. 5c). Activation time constants were voltage-dependent for both LVA and HVA barium currents, being faster at more positive potentials $\left(\tau_{\text {act }}\right.$ at $-57 \mathrm{mV}$ and $+3 \mathrm{mV}$, LVA, $1.11 \pm 0.18 \mathrm{~ms}$ and $0.53 \pm 0.09 \mathrm{~ms}$; HVA, $2.77 \pm 0.76 \mathrm{~ms}$ and $0.78 \pm 0.12 \mathrm{~ms}$ respectively, $n=6, P<0.05$ for both comparisons, Fig. $5 \mathrm{~d}$ ). There was also significant difference between LVA and HVA activation at all holding potentials more negative than $-7 \mathrm{mV}$ but not at more positive potentials ( $n=6, P<0.05$, paired t-test). Mean activation time constant $\mathrm{V}_{50}$ for LVA and HVA barium currents was $-49.2 \pm 3.4 \mathrm{mV}$ and $-39.7 \pm 5.1 \mathrm{mV}$ with slope values of $-5.8 \pm 3.0$ and $-13.4 \pm 5.1$ respectively $(n=6$, Fig. 5d). LVA inactivation time constant exhibited voltage-dependency, being slower at more positive potentials (at $-67 \mathrm{mV}$, $8.5 \pm 0.8 \mathrm{~ms}$; at $+3 \mathrm{mV}, 16.8 \pm 1.4 \mathrm{~ms}, n=6, P<0.05$, paired ttest, Fig. 5e), while when fitted with a Boltzmann function in the voltage range it exhibited $\mathrm{V}_{50}$ value of $-34.5 \pm 11.1 \mathrm{mV}$ with slope of $0.37 \pm 4.3(n=6)$. Fast voltage-ramps $(200-500 \mathrm{mV} / \mathrm{s}$, from $-107 \mathrm{mV}$ to $+13 \mathrm{mV}$ ) recruited a barium current peaking at -20 to $-30 \mathrm{mV}$ but were less successful in isolating a distinct peak reflecting an LVA component as seen in a some neurons recorded in calcium (e.g. Figure 4d). Despite this we found evidence of activation of an inward conductance at potentials more negative than $-60 \mathrm{mV}$ (Fig. 5f) of different slope than the activation of the HVA component that is expected to begin at much more positive potentials (starting at around $-35 \mathrm{mV}$ ). Steady-state activation $\mathrm{V}_{50}$ for the background voltage-ramp barium current was $-38.9 \pm 2.5 \mathrm{mV}$ with slope value of $6.3 \pm 1.5(n=4)$. Interestingly, leak current reversal during these voltage ramps appeared $30 \mathrm{mV}$ more positive than ramps conducted in calcium (compare ramps in Fig. 4d and 5f) indicating that the leak conductance is probably at least partially carried forward to a degree by potassium ions through bariumsensitive ion channels. Nimodipine $(10 \mu \mathrm{M})$, a L-type calcium channel blocker, blocked partially the HVA component (30-40\% block) evoked by depolarizing steps (from $-87 \mathrm{mV}$ in $10 \mathrm{mV}$ increments) without affecting the transient LVA component signifying that at least in part HVA barium currents are carried forward via the L-type calcium channel (online resource 4A, B).

\subsection{Sodium $\left(\mathrm{I}_{\mathrm{Na}}\right)$ currents}

Transient and background 'persistent' sodium currents are operant on VTA and SNc neurons contributing differentially on their autorhythmicity (Puopolo et al. 2007; Khaliq and Bean 2010). We have recorded fast activating/inactivating $\left(\mathrm{I}_{\mathrm{NaT}}\right)$ and persistent/non-inactivating inward sodium currents $\left(\mathrm{I}_{\mathrm{NaP}}\right)$ through a standard activation depolarization step protocol and 

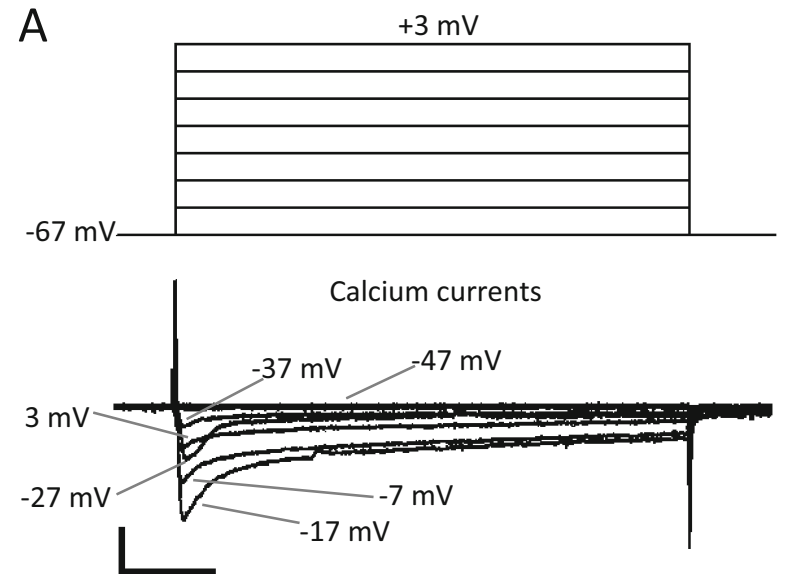

B

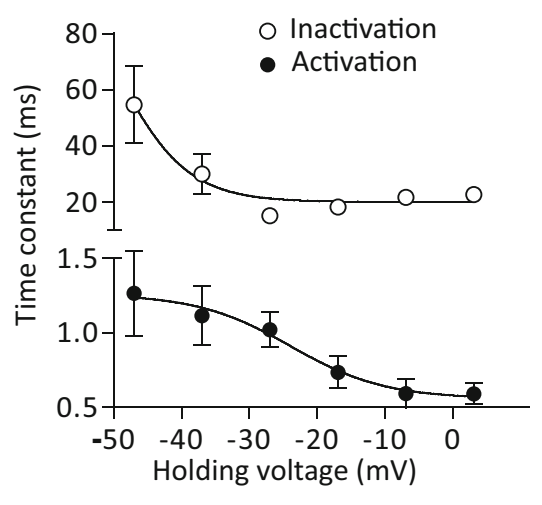

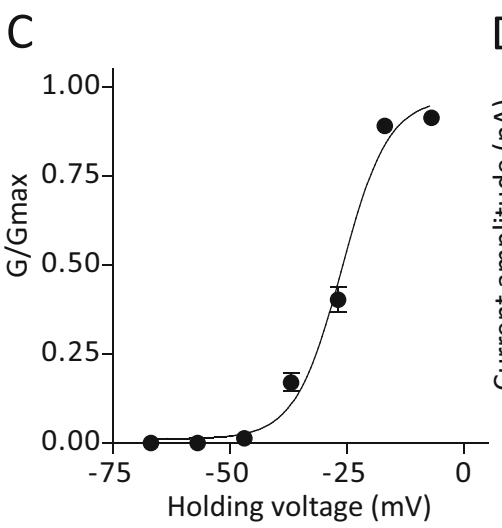

$\mathrm{F}$

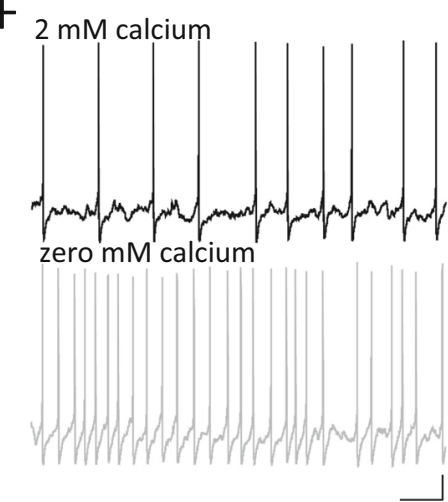

D
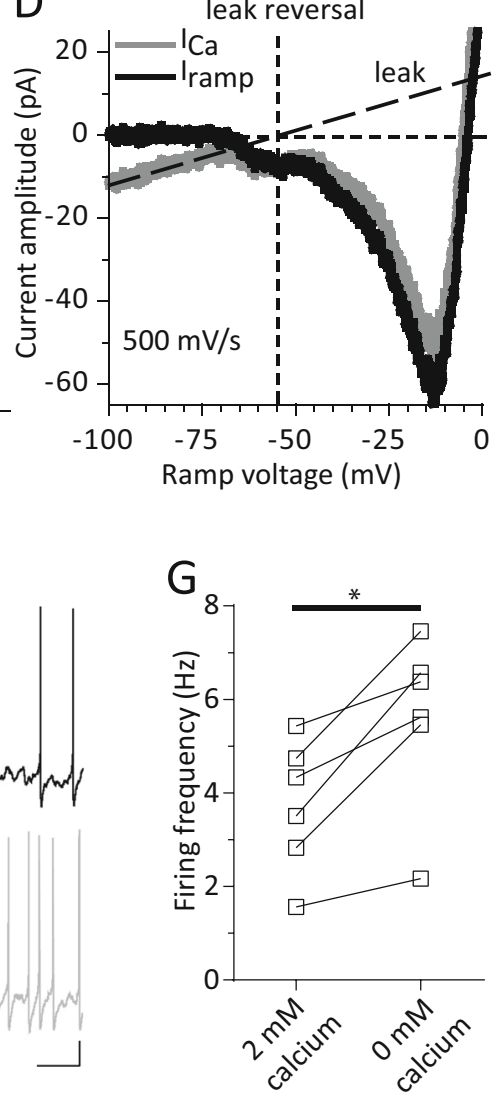

E

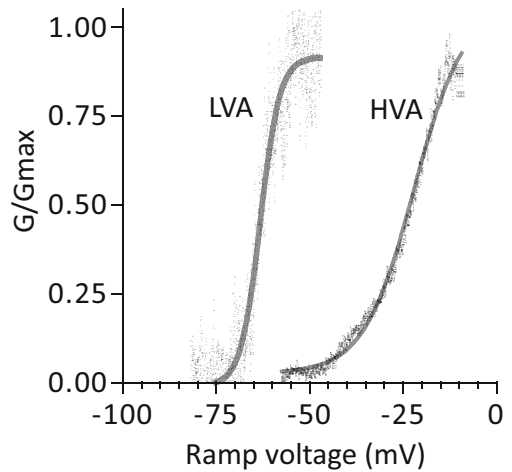

$\mathrm{H}$

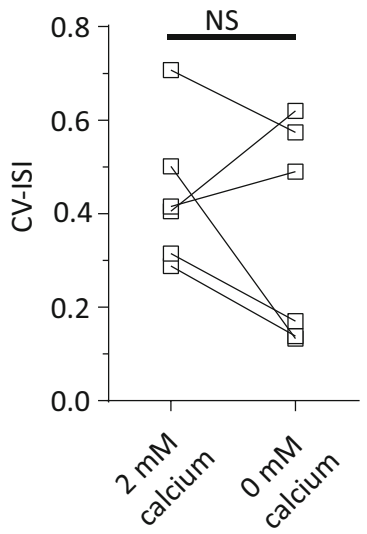

voltage-ramps in the presence of cadmium, cesium and TEA to block calcium and potassium currents respectively as reported previously (Pignatelli et al. 2005; Magistretti et al. 2006). Most voltage-clamped vlPAG/DRN DA neurons showed typical signs of problematic space-clamp control upon recordings of the fast transient sodium conductance as reported previously for neurons in brain slices (e.g. see Magistretti et al. 2006). Typical signs included distortion of sodium current waveform, clear long latencies to spike (> $4 \mathrm{~ms}$ from step onset to peak) and non-graded responses in activation implying activation of unclamped axonic sodium channels. To overcome this problem we have used a modified protocol exploiting a prepulse to selectively inactivate axonal currents and resolve somatic sodium currents as described previously (Milescu et al. 2010). Cells were held at $-107 \mathrm{mV}$ and a $4 \mathrm{~ms}$ depolarizing pulse to $-47 \mathrm{mV}$ was delivered to activate unclamped sodium channels (somatic and axonic, Milescu et al. 2010). Then, a 5 ms hyperpolarizing pulse was delivered to $-77 \mathrm{mV}$ to facilitate the fast deactivation of somatic (but not axonal, see Milescu et al. 2010) sodium currents before delivering $110 \mathrm{~ms}$ long, incremental $5 \mathrm{mV}$ step depolarizations (up to $+13 \mathrm{mV}$ ) to record somatic sodium currents. We routinely recorded good quality somatic sodium 
4 Fig. 4 Calcium currents $\left(\mathrm{I}_{\mathrm{Ca}}\right)$. a. Typical electrophysiological traces of inward calcium currents recorded using a series of depolarizing test steps ( $250 \mathrm{~ms}$ duration, holding potential $-67 \mathrm{mV}$, pulses from -67 to $+3 \mathrm{mV}$, $10 \mathrm{mV}$ increments) in the presence of TTX, 4-AP and TEA (see methods). Note that the calcium currents inactivate during the long test step (scale bars, $50 \mathrm{pA}$ and $50 \mathrm{~ms}$ ). b. Voltage-dependence of activation and inactivation time constants $\left(\tau_{\text {act }}\right.$ and $\left.\tau_{\text {ina }}\right)$ of recorded calcium currents (as shown in A, $n=4)$. To measure $\tau_{\text {act }}$ and $\tau_{\text {ina }}$ time constants calcium currents were fitted a single exponential function (start to peak and peak to end respectively) at each holding potential. Both $\tau_{\text {act }}$ and $\tau_{\text {ina }}$ were voltagedependent, becoming faster at more positive potentials (mean $\tau_{\text {act }}$ and $\tau_{\text {ina }}$ at $-47 \mathrm{mV}, 1.27 \mathrm{~ms}$ and $114 \mathrm{~ms}$; at $-17 \mathrm{mV}, 0.62 \mathrm{~ms}$ and $16.7 \mathrm{~ms}$ respectively). Plotted data were then fitted with a single Boltzmann function to calculate mean $\mathrm{V}_{50}$ (activation, $-23.8 \mathrm{mV}$; inactivation, $-39.0 \mathrm{mV}$ ) and slope (LVA, -6.9 ; HVA, -2.6$)$ values $(n=4)$. c. Average steady-state activation curve $\left(\mathrm{G} / \mathrm{G}_{\max }\right)$ for calcium currents recorded using a series of depolarising pulses (as shown in A). Normalised conductance plot was fitted with a single Boltzmann function to calculate mean $\mathrm{V}_{50}$ $(-26.1 \mathrm{mV})$ and slope $(5.0)$ values for steady state activation $(n=4)$. d. Fast voltage-ramp $(500 \mathrm{mV} / \mathrm{s},-107$ to $+13 \mathrm{mV})$ depicting the activation of a calcium currents. Two distinct peaks were identified (both sensitive to cadmium, not shown) that indicate that LVA and HVA calcium currents are expressed on this vlPAG/DRN neuron. Leak subtracted current (dotted line) revealed that LVA calcium currents peaked at around $-60 \mathrm{mV}$ and HVA calcium currents peaked at around $-15 \mathrm{mV}$. Leak current reversal under these conditions was $-55 \mathrm{mV}$. e. Steady-state activation curves $\left(\mathrm{G} / \mathrm{G}_{\max }\right)$ for LVA and HVA calcium currents for the neuron shown in D. Normalised conductance plots were fitted with a single Boltzmann function to calculate $\mathrm{V}_{50}$ (LVA, $-63.2 \mathrm{mV}$; HVA, $-22.1 \mathrm{mV}$ ) and slope (LVA, 2.6; HVA, 6.7) values for steady-state activation. f. Representative electrophysiological traces recorded in current-clamp mode in normal $(2 \mathrm{mM}$ ) or zero calcium (see methods) aCSF. Substitution of magnesium with calcium increased spontaneous firing of vlPAG/DRN DA neurons without affecting the firing rate regularity (scale bars, $10 \mathrm{mV}$ and $2 \mathrm{~s}$ ). g. Bar chart comparison of mean firing frequency before and after replacement of $2 \mathrm{mM}$ calcium for magnesium as shown in $\mathrm{F}$ for seven vlPAG/DRN DA neurons. This replacement caused a statistically significant change in firing rate (mean firing frequency in $2 \mathrm{mM}$ calcium, $3.3 \mathrm{~Hz}$; in zero $\mathrm{mM}$ calcium, $4.9 \mathrm{~Hz}, n=6,{ }^{*} P<0.05$, paired t-test). h. Bar chart comparison of CV-ISI before and after replacement of $2 \mathrm{mM}$ calcium for magnesium as shown in $\mathrm{F}$ for seven vlPAG/DRN DA neurons. This replacement did not cause a statistically significant change in CV-ISI (mean CV-ISI in $2 \mathrm{mM}$ calcium, 0.66 ; in zero $\mathrm{mM}$ calcium, $0.40, n=6, P>0.05$, paired t-test, NS, not significant)

currents in one every 3-4 neurons at room temperature (20 $22{ }^{\circ} \mathrm{C}$ ) but at a much lower yield of one every 8-10 neurons at higher temperature in line with the problems that occur in electrophysiological recordings of very fast activating currents (Hille 2001; Milescu et al. 2010). At our target temperature of $35^{\circ} \mathrm{C}$, a few vlPAG/DRN DA neurons ( $n=6$, from three mice) exhibited a smooth graded response of somatic sodium current activation in the subthreshold range of -65 to $-40 \mathrm{mV}$ were accepted for kinetic analysis (Fig. 6a). Steady-state inactivation was studied by a series of prepulses ( -77 to $-42 \mathrm{mV}$ for $5 \mathrm{~ms}$, test pulse at $-37 \mathrm{mV}$ for $20 \mathrm{~ms}$ ) delivered after a depolarizing step ( -107 to $-47 \mathrm{mV}$ for $4 \mathrm{~ms}$ ) to induce the inactivation of the axonal sodium current as detailed above for the construction of the steady-state activation curve (Fig. 6b). Following the fast transient sodium response, a "persistent", background, sodium current appeared as an inward non-inactivating/slowly inactivating current upon $110 \mathrm{~ms}$ long step depolarizations (Fig. 6c). Both fast and persistent currents were fully sensitive to TTX while we did not detect any consistent residual inward current in the presence of TTX that would imply the activation of a TTX-resistant sodium conductance $(n=8$, from four mice, data not shown). Slow voltage-ramps $(16 \mathrm{mV} / \mathrm{s},-107$ to $+53 \mathrm{mV}$ ) confirmed the presence of a background sodium conductance (Fig. 6d) that was 100-150 times smaller in magnitude than the transient sodium conductance. The average $G_{\max }$ for the transient sodium component was $16.1 \pm 1.2 \mathrm{nS}$ with $\mathrm{G}_{\max }$ of persistent sodium currents reaching a much smaller value of $0.19 \pm 0.04 \mathrm{nS}(n=6)$. Step depolarization and slow voltage-ramp activation protocols resulted in a $\mathrm{G}_{\mathrm{NaP}}$ that activated 5-10 $\mathrm{mV}$ more negative than $\mathrm{G}_{\mathrm{NaT}}$ (Fig. 6e). Steady-state activation $\mathrm{V}_{50}$ values were $-45.2 \pm 1.4 \mathrm{mV}$ and $-56.6 \pm 1.3 \mathrm{mV}$ with slope of $5.3 \pm 1.3$ and $3.3 \pm 1.0$ for $\mathrm{G}_{\mathrm{NaT}}$ and $\mathrm{G}_{\mathrm{NaP}}$ respectively ( $n=6$ ) whereas the corresponding values for ramp voltage determination of $\mathrm{G}_{\mathrm{NaP}}$ for $\mathrm{V}_{50}$ and slope was $-60.7 \pm 3.0 \mathrm{mV}$ and $2.3 \pm 0.7(n=3$, Fig. $6 \mathrm{e})$. $\mathrm{I}_{\mathrm{NaT}}$ steadystate inactivation had a $\mathrm{V}_{50}$ of $-62.8 \pm 2.5$ and a slope of $-6.4 \pm 1.7$ ( $n=4$, Fig. 6e). $\mathrm{I}_{\mathrm{NaT}}$ activation time constant $\left(\tau_{\text {act }}\right)$ was voltage-dependent becoming faster at more positive potentials $\left(\tau_{\text {act }}\right.$ at $-47 \mathrm{mV}, 704.5 \pm 152.4 \mu \mathrm{s}$; at $-2 \mathrm{mV}$, $147.2 \pm 17.8 \mu \mathrm{s}, n=6, P<0.05$, paired t-test, Fig. $6 \mathrm{f})$. The $\mathrm{V}_{50}$ and slope for the $\tau_{\text {act }}$ was $-34.4 \pm 4.1 \mathrm{mV}$ and $-5.6 \pm 3.8$ respectively $(n=6)$. The inactivation phase of the sodium current was consistently fitted with two (fast and slow, $\tau_{\text {inaF }}$ and $\left.\tau_{\text {inas }}\right)$ rather than one exponential function. The contribution of the $\tau_{\text {inas }}$ exponential component in the inactivation phase of $\mathrm{I}_{\mathrm{NaT}}$ would account only for $<5 \%$ of the amplitude of the current for the voltage range studied (up to $-2 \mathrm{mV}, n=6$, data not shown). $\mathrm{I}_{\mathrm{NaT}}$ inactivation time constants showed similar voltage-dependence (Fig. $6 \mathrm{f}$ ) with both $\tau_{\text {inaF }}$ and $\tau_{\text {inas }}$ becoming faster at more positive potentials $\left(\tau_{\text {inaF }}\right.$ and $\tau_{\text {inas }}$ at $-47 \mathrm{mV}$, $888.8 \pm 83 \mu \mathrm{s}$ and $17.4 \pm 2.0 \mathrm{~ms}$; at $-2 \mathrm{mV}, 410.2 \pm 80.5 \mu \mathrm{s}$ and $4.2 \pm 0.6 \mathrm{~ms}$ respectively, $n=6, P<0.05$ for both paired comparisons, paired t-test). The corresponding $\mathrm{V}_{50}$ values for $\tau_{\text {inaF }}$ and $\tau_{\text {inas }}$ extracted with a first order Boltzmann in the voltage range tested, returned values of $-46.4 \pm 1.5 \mathrm{mV}$ and $-50.5 \pm 1.4 \mathrm{mV}$ and slopes of $-11.3 \pm 1.5$ and $-7.7 \pm 1.4$ respectively $(n=6)$. To investigate the impact of sodium channel blockade on spontaneous firing and pacemaker frequency we recorded in current-clamp in control conditions using standard KGlu filled electrodes and superfused the slice with $1 \mu \mathrm{M}$ TTX (Fig. 6g, h) as reported previously (Khaliq and Bean 2010). We found that after perfusion of TTX the neurons stopped firing and had a stable, non-oscillating, resting membrane potential that was on average $-6.4 \pm 0.8 \mathrm{mV}$ more negative than the average action potential threshold $(-43.1 \pm 1.3 \mathrm{mV})(n=8$, from three mice, data not shown). These data suggest that TTX-sensitive sodium currents are important in membrane repolarization to AP threshold and neuron's spontaneous firing. 
A

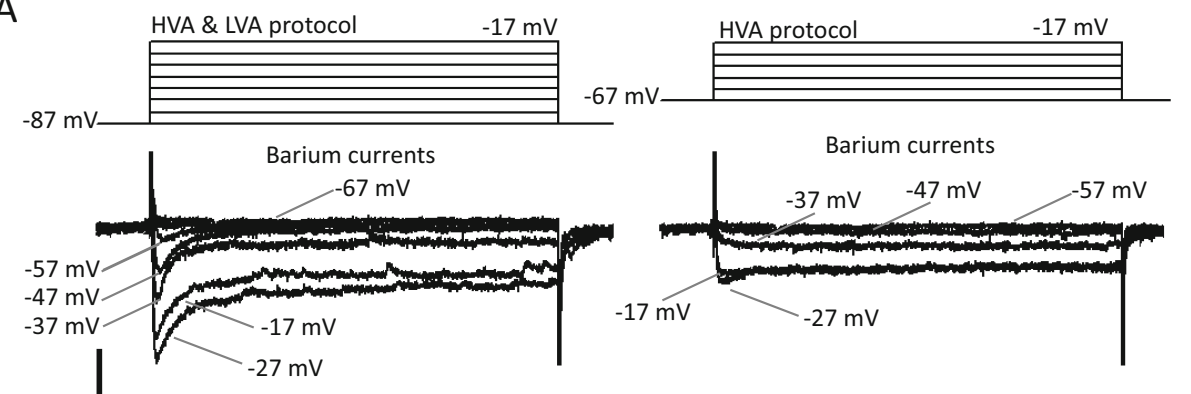

C

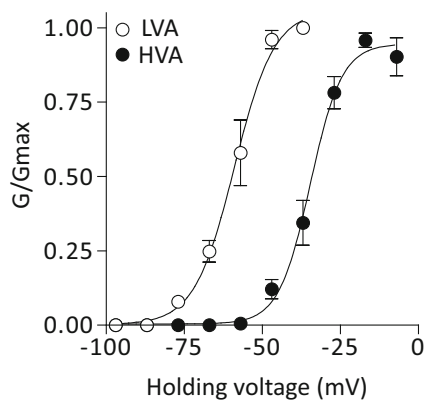

D

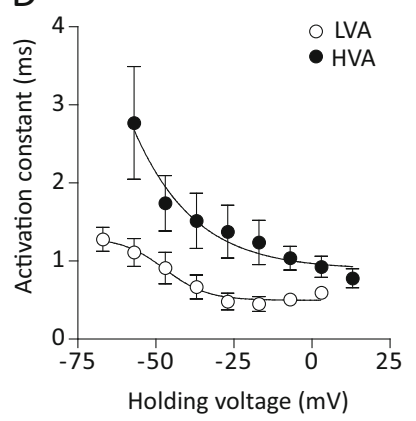

Fig. 5 Barium currents $\left(\mathrm{I}_{\mathrm{Ba}}\right)$. a. Typical electrophysiological traces of inward barium currents recorded using two different protocols (differing in their starting holding voltage) for isolating composite LVA/HVA (left) and HVA only (right) barium currents (see methods). Note the transient barium currents in the LVA/HVA protocol and the non-inactivating barium currents in the HVA protocol during the long test step (scale bars, $25 \mathrm{pA}$ and $50 \mathrm{~ms}$ ). b. Typical electrophysiological traces from HVA/LVA composite and HVA only protocols at a single potential of $-17 \mathrm{mV}$ taken from A. Subtraction of the current traces revealed the LVA transient component. c. Average steady-state activation curves $\left(\mathrm{G} / \mathrm{G}_{\max }\right)$ for LVA and HVA barium currents. Normalised conductance plots were fitted with a single Boltzmann function to calculate mean $\mathrm{V}_{50}$ (LVA, $-58.9 \mathrm{mV}$; HVA, $-34.6 \mathrm{mV}$ ) and slope (LVA, 6.3; HVA, 5.1) values for activation $(n=6)$. d. Voltage-dependence of barium LVA and HVA current activation time constant. HVA and subtracted LVA currents were fitted a single exponential function (start to peak) at each test holding voltage to calculate $\tau_{\text {act }}$ for each component. Both HVA and LVA had a $\tau_{\text {act }}$ that was voltage-dependent becoming faster with more positive potentials (mean LVA and HVA $\tau_{\text {act }}$ at $-47 \mathrm{mV}, 0.91 \mathrm{~ms}$ and $1.74 \mathrm{~ms}$; at $+3 \mathrm{mV}, 0.53 \mathrm{~ms}$ and $0.78 \mathrm{~ms}$ respectively). Plotted data fitted with a single Boltzmann function to calculate mean $\mathrm{V}_{50}$ (LVA, $-49.2 \mathrm{mV}$; HVA, $-39.7 \mathrm{mV}$ ) and slope (LVA, -5.8 ; HVA, -13.4$)$ values $(n=6)$. e. Voltage-dependence of barium LVA current inactivation time constant. Subtracted LVA currents were fitted a single exponential function (peak to

\subsection{Model DA neuron}

\subsubsection{Validation of in silico model DA neuron}

Based on our experimental data, we constructed a single compartment, simplified, Hodgkin and Huxley- type DA neuron to compare model and in vitro neurophysiological behavior and explore the origin of autorhythmicity and pacemaking in silico. A summary of the mean values used for constructing the model DA neuron are given in Table 1 . General equations used for the model DA neuron are given in the appendix. The
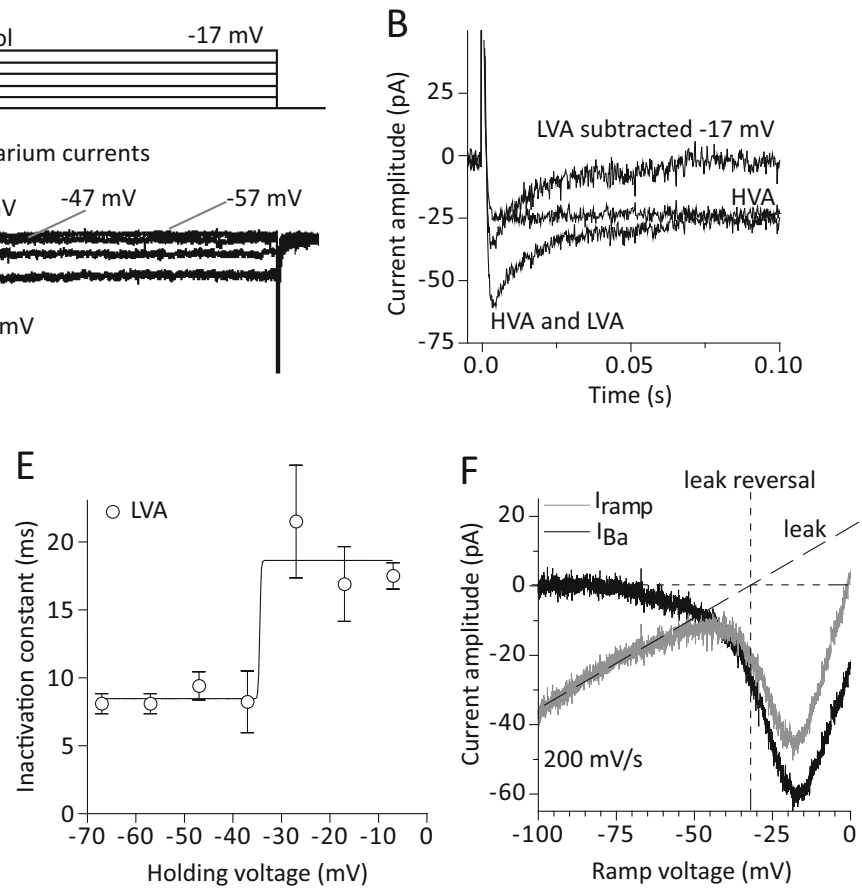

end) at each test holding voltage to calculate the inactivation time constant $\left(\tau_{\text {ina }}\right)$. Inactivation time constant became slower at more positive voltages (mean $\tau_{\text {ina }}$ at $-47 \mathrm{mV}$ and $+3 \mathrm{mV}, 8.5 \mathrm{~ms}$ and $16.8 \mathrm{~ms}$ respectively). Plotted data were fitted with a single Boltzmann function to calculate the mean $\mathrm{V}_{50}(-34.5 \mathrm{mV})$ and slope $(0.37)$ values for $\tau_{\text {ina }}$ $(n=6)$. f. Fast voltage-ramp $(200 \mathrm{mV} / \mathrm{s},-107$ to $+13 \mathrm{mV})$ taken from the cell shown in A depicting the activation of a background barium current. Leak subtracted current (dotted line) revealed that barium currents peaked at around $-20 \mathrm{mV}$. Fast voltage-ramps in barium were less efficient than voltage steps in isolating the LVA transient (see also Fig. 4d, e for calcium voltage-ramps). Although we did not observe a well-defined peak at the voltage range of -65 to $-55 \mathrm{mV}$ we observed the development of an inward current at such potentials well before the expected start of the activation of HVA current (usually around $-35 \mathrm{mV}$, peaking at -15 to $-20 \mathrm{mV}$ ). Note the change in the slope of the development of the putative LVA and HVA inward barium currents. Plotted voltage-ramp data were fitted with a single Boltzmann function to calculate the mean $\mathrm{V}_{50}(-38.9 \mathrm{mV})$ and slope (6.3) values for barium current steady-state activation. Note that, in the presence of barium, leak current is reversing at around $-25 \mathrm{mV}$ which is $30 \mathrm{mV}$ more positive that its reversal when using calcium as the charge carrier suggesting that a barium sensitive leak conductance is operant on vlPAG/DRN DA neurons

model DA neuron exhibited autorhythmicity and fired APs at a pacemaker frequency of $4.6 \mathrm{~Hz}$ (Fig. 7a). AP characteristics (Fig. 7b) were measured and were compared with the corresponding mean values measured in the in vitro DA neuron in brain slices (Table 2, Fig. 7b). We found that the model DA neuron had similar AP characteristics and internal ratios to the native DA neurons (see also Dougalis et al. 2012) but notably had a faster action potential in width (at base) than in vitro vlPAG/DRN DA neurons. Model DA neuron exhibited an input resistance of $1.3 \mathrm{G} \Omega$ when subjected to small $(-10 \mathrm{pA}$, $1000 \mathrm{~ms}$ ) hyperpolarizing current injection in range with 
in vitro data (Dougalis et al. 2012). We injected a series of hyperpolarizing and depolarizing currents to the model DA neuron to validate its electrophysiological behavior (Fig. 7c, d) and to compare and contrast it with that of the in vitro DA neuron (Fig. 7e-h). Hyperpolarizing current injections were used to monitor the hyperpolarization-activated, cation current ( $\mathrm{I}_{\mathrm{H}}$ current-mediated voltage-sag) and $\mathrm{I}_{\mathrm{A}}$ current-mediated delayed repolarization (quantified as the delay to firing a spike after the end of a hyperpolarizing current injection, Fig. 7c) which are the hallmarks of DA neuron electrophysiological behavior in vlPAG/DRN. Both responses were qualitatively and quantitatively similar to these obtained from in vitro brain slice preparation (Fig. $7 \mathrm{~g}, \mathrm{~h}$ ). Notably, the responses to the voltage-sag amplitude appeared fairly linear to those in vitro but the responses of the delayed depolarization produced an abruptly graded response at low current injections with no further increases detectable in delay to spiking thereafter. Upon injection of depolarizing pulses (Fig. 7d), the model DA neuron exhibited responses with similar gain (slope) in firing output to in vitro DA neurons only when given small magnitude current injection (10-40 pA, Fig. 7e-f). However, upon increasing the magnitude of the current injection (60$120 \mathrm{pA}$ ), the model DA neuron exhibited higher gain in output and fired at higher frequencies than that of the in vitro DA neurons while, it exhibited depolarization block (DB) at current magnitude within a similar range with in vitro data (Fig. 7e, f). Such behavior could be improved dramatically when needed by using a range of values reflecting the mean \pm one standard deviation of the experimentally derived parameter during optimization without changing qualitatively the behavior and the origins of pacemaker (see later Figs. 8-10). These data indicate that despite its apparent reductionist approach in the modelling of ion channel properties imposed herein, the model DA neuron captured and replicated well essential elements of vlPAG/DRN DA neuronal behavior as seen in vitro. Using this basal state of the model without further adjustments we then proceeded into further experiments attempting to understand the contribution of individual ion currents to firing frequency, autorhythmicity and threshold for depolarization block (Figs. 8-10).

\subsubsection{Contribution of ionic conductances in pacemaker firing frequency and expression of autorhythmicity}

To evaluate the contribution of each ionic current to pacemaker firing frequency and neuronal autorhythmicity we used the model DA neuron to generate predictions by reducing the maximal conductance levels $\left(\mathrm{G}_{\max }\right.$ from $100 \%$ to zero) of each one individual conductance in turn (Fig. 8). We found that complete elimination of the $\mathrm{I}_{\mathrm{A}}$ current caused a two-fold increase of model DA neuron basal pacemaker frequency (Fig. 8b). In contrast, eliminating the $\mathrm{I}_{\mathrm{H}}$ current lead to a very small change (decrease) in firing frequency (Fig. 8c).
Eliminating $\mathrm{I}_{\mathrm{CaLVA}}$ and $\mathrm{I}_{\mathrm{CaHVA}}$ calcium conductances decreased the model's pacemaking frequency to different levels (Fig. 8d, e). The former, but not the latter, appeared as a strong regulator of firing frequency (causing an approximate $25 \%$ reduction in basal firing frequency) but complete and concomitant elimination of both calcium conductances did not stop spontaneous firing suggesting that calcium currents are not indispensable for vlPAG/DRN neuron's spontaneous firing (Fig. 8f). Complete elimination of $\mathrm{I}_{\mathrm{M}}$ current did not affect autorhythmicity either but instead it caused a two-fold increase in firing frequency (Fig. 8g). In contrast, small reductions of $I_{K d r}$ initially increased firing frequency (data not shown) but when the $G_{\max }$ value was reduced below $25 \%$ of its basal level, a stable non-oscillating depolarized potential above AP threshold $(-35 \mathrm{mV})$ was attained accompanied by complete elimination of spontaneous firing even upon depolarizing current injections (Fig. 8h). Under these circumstances, even a small increase in the magnitude of the sustained outward $\mathrm{I}_{\mathrm{M}}$ current (to $125 \%$ of the basal values, see online resource 3, also Wladyka and Kunze 2006; Shah et al. 2011) could rescue firing even when $I_{K d r}$ was set to zero (Fig. 8i) suggesting that although the $\mathrm{I}_{\mathrm{kdr}}$ current modulates strongly autorhythmicity, its effect could be rescued by the small increase of another sustained potassium conductance in line with what would be expected from a Hodgkin and Huxley formalism, where the operation of sustained potassium conductances is a prerequisite for spontaneous firing. Elimination of $\mathrm{I}_{\mathrm{NaP}}$ obliterated spontaneous firing but APs could still be observed under injection of depolarizing current (Fig. 8j). Eliminating the $\mathrm{I}_{\mathrm{NaT}}$ current alone halted spontaneous firing (and depolarizing current injection- induced firing) but caused the appearance of a $6 \mathrm{mV}$ oscillating background (at a frequency of $5.1 \mathrm{~Hz}$ ) below AP threshold. Interestingly, concomitant elimination of both transient and persistent sodium currents stopped firing and produced a stable resting membrane potential nearly $10-20 \mathrm{mV}$ more negative from the AP threshold ( -55 to- $64 \mathrm{mV}$, depending on the simulation conditions, Fig. $8 \mathrm{k}$ ). The origin of the background oscillating potential seen in the absence of a $\mathrm{I}_{\mathrm{NaT}}$ current was explored by inducing small reductions (to $75 \%$ of the value of $\mathrm{G}_{\max }$ ) in either the $\mathrm{I}_{\mathrm{NaP}}$ current (Fig. 8m) or the $\mathrm{I}_{\mathrm{M}}$ current (Fig. 8n). Both treatments completely obliterated the background oscillation observed in the absence of the $\mathrm{I}_{\mathrm{NaT}}$ current suggesting an interaction of the $\mathrm{I}_{\mathrm{M}}$ and $\mathrm{I}_{\mathrm{NaP}}$ persistent and opposing currents could give rise to a background oscillation under certain values/conditions in the subthreshold range when the $\mathrm{I}_{\mathrm{NaT}}$ current is completely eliminated. These simulation data suggest that sodium, but not calcium, currents are primarily the mediators of autorhythmicity in the model DA neuron, while any individual potassium current alone $\left(\mathrm{I}_{\mathrm{A}}, \mathrm{I}_{\mathrm{Kdr}}, \mathrm{I}_{\mathrm{M}}\right)$, although strongly modulated pacemaker firing frequency, did not endow DA neurons with autorhythmicity. In contrast, it is a prerequisite that all individual sustained potassium currents 

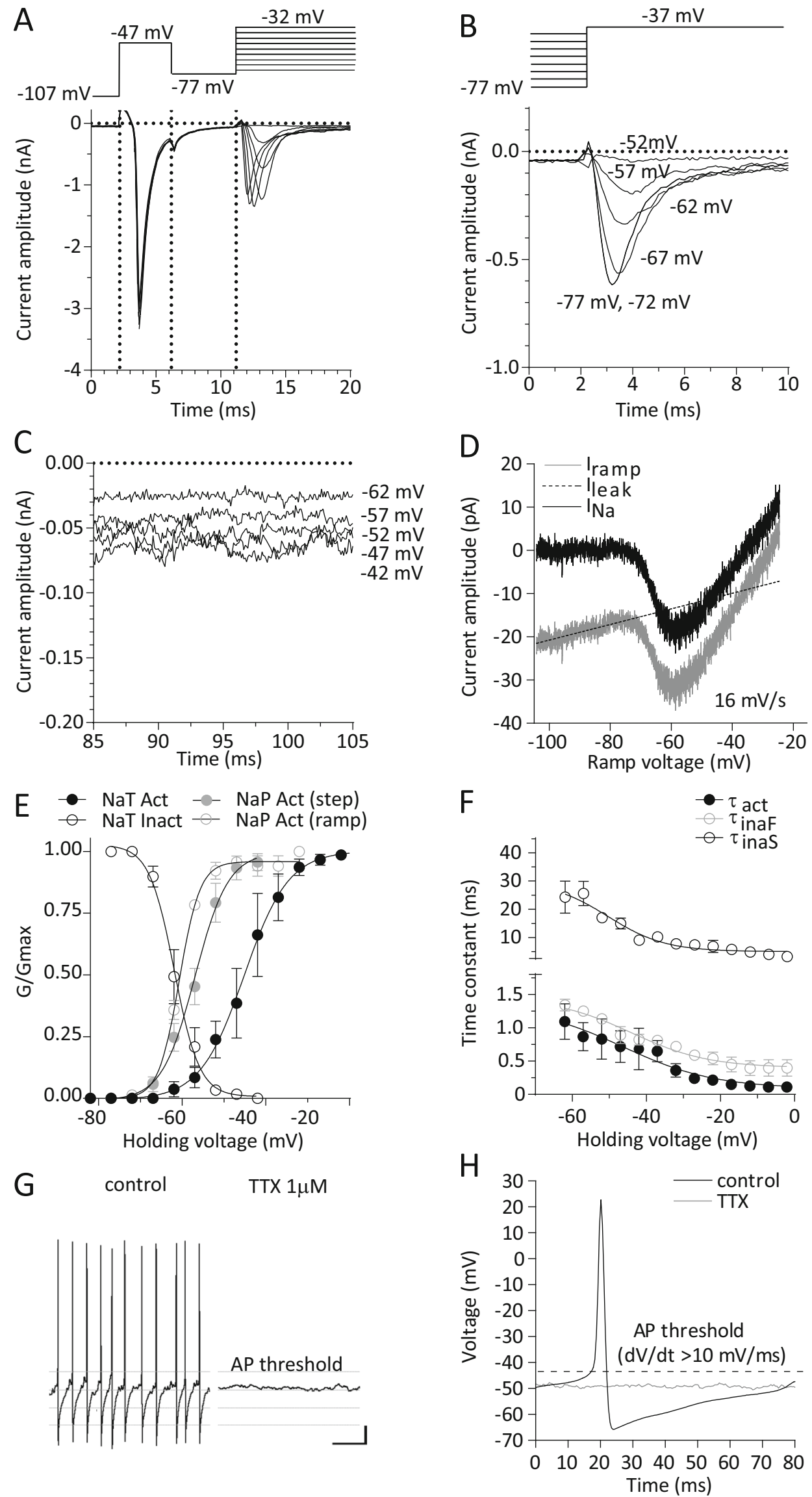
4Fig. 6 Transient and persistent sodium currents $\left(\mathrm{I}_{\mathrm{NaT}}\right.$ and $\left.\mathrm{I}_{\mathrm{NaP}}\right)$. a. Representative average electrophysiological traces depicting the activation of a transient inward sodium current recorded using a protocol that selectively inactivates axonal sodium currents as described previously (Milescu et al. 2010). Neurons were held at $-107 \mathrm{mV}$ and a depolarising pulse ( $4 \mathrm{~ms}$ ) was delivered to $-47 \mathrm{mV}$ to elicit unclamped sodium currents, followed by a brief $5 \mathrm{~ms}$ hyperpolarisation to $-77 \mathrm{mV}$ to facilitate recovery of somatic (but not axonal) sodium currents before eliciting a series of test pulses in $5 \mathrm{mV}$ increments and of $110 \mathrm{~ms}$ in duration to activate somatic sodium currents. Experiments were conducted in the presence of blockers of potassium and calcium conductances. Note the activation of the unclamped distorted sodium current in the initial depolarising $5 \mathrm{~ms}$ step and the gradual incremental nature of the sodium currents during the test pulse. b. Representative average electrophysiological traces taken from cell shown in A depicting the protocol used to study steady-state inactivation of somatic sodium channels. Holding current and initial depolarisation to activate unclamped sodium currents were identical to the one used for the study of steady-state activation (shown in A) but the subsequent $5 \mathrm{~ms}$ hyperpolarising pulse varied from $-77 \mathrm{mV}$ to $-42 \mathrm{mV}$ while the test pulse for studying the inactivation was set constant to $-37 \mathrm{mV}$. c. Representative average electrophysiological traces taken from cell shown in A depicting the persistence of an inward current even after $100 \mathrm{~ms}$ of depolarization at different potentials. Test potential are displayed next to each trace. Traces displayed here have not been subtracted for linear leak current. The transient sodium current occurring at the beginning of the traces have been truncated for simplicity. d. Slow voltage-ramp $(16 \mathrm{mV} /$ $\mathrm{s},-107$ to $+53 \mathrm{mV}$ ) depicting the activation of a background persistent sodium current. Leak subtracted current (dotted line) revealed the activation kinetics of the persistent sodium current $\left(\mathrm{I}_{\max }\right.$ of $15 \mathrm{pA}$ at $-57 \mathrm{mV}$ ). e. Average steady-state activation/inactivation curves $\left(\mathrm{G} / \mathrm{G}_{\max }\right)$ for the transient and persistent sodium current $(n=6)$. Persistent sodium current curves have been quantified via both a prepulse step protocol shown in A and $\mathrm{C}(n=6)$ and through slow voltage ramps as shown in $\mathrm{D}(n=3)$. Normalised conductance plots were fitted with a single Boltzmann function to calculate mean steady-state activation $\mathrm{V}_{50}$ (transient, $-45.2 \mathrm{mV}$;

are blocked simultaneously or reduced to less than $10 \%$ of their basal $G_{\max }$ value that causes spontaneous firing to cease due to the attainment of a depolarized steady state (e.g. Figure 8h).

\subsubsection{Contribution of ionic conductances in repolarization during ISI}

To understand in more detail the interplay of ionic currents in pacemaking and their contribution to the repolarization during the ISI we examined the trajectories of the each ionic current during this period in the model DA neuron (Fig. 9). We found that $\mathrm{I}_{\mathrm{kdr}}$ potassium currents deactivated with an outward relaxation during ISI facilitating repolarization while the $\mathrm{I}_{\mathrm{A}}$ and $\mathrm{I}_{\mathrm{M}}$ currents activated during ISI thus opposing repolarization (Fig. 9b). Interestingly, the $\mathrm{I}_{\mathrm{M}}$ current did not fully inactivate exhibiting a persistent outward current even at the peak of the AHP. We detected a rather small contribution (compared to $\mathrm{I}_{\mathrm{Kdr}}$ and $\mathrm{I}_{\mathrm{A}}$ type currents) from a inward current relaxation from the $\mathrm{I}_{\mathrm{H}}$ current giving rise to a net outward current during ISI repolarisation. Importantly, $\mathrm{I}_{\mathrm{CaHVA}}$ current did not contribute with any inward current during the ISI repolarization but $\mathrm{I}_{\mathrm{CaLVA}}$ current gave rise to a small inward persistent current persistent, $-56.6 \mathrm{mV}$ ) and its slope (transient, 5.3; persistent, 3.3). Ramp voltage determination of persistent sodium current activation had a mean $\mathrm{V}_{50}$ and slope of -60.7 and 2.3 respectively. Mean transient sodium current inactivation $V_{50}$ and slope values were $-62.8 \mathrm{mV}$ and -6.4 respectively. $\mathbf{f}$. Voltage-dependence of $\mathrm{I}_{\mathrm{NaT}}$ current activation and inactivation time constants. $\mathrm{I}_{\mathrm{NaT}}$ currents were fitted a single exponential function (start to peak) and two exponential functions (from peak to end) at each test holding voltage to calculate a single activation $\left(\tau_{\text {act }}\right)$ and two $\left(\tau_{\text {inaF }}\right.$ and $\left.\tau_{\text {inas }}\right)$ inactivation time constants respectively. Activation time constant appeared voltage-dependent becoming faster at more positive potentials (mean $\tau_{\text {act }}$ of $705 \mu \mathrm{s}$ at $-47 \mathrm{mV}$ and $147 \mu$ s at $-2 \mathrm{mV}$ ). Data were plotted against holding voltage and fitted with a single Boltzmann function to calculate mean $\mathrm{V}_{50}(-34.4 \mathrm{mV})$ and slope $(-5.6)$ values $(n=6)$. Inactivation was consistently better fitted with two rather than one exponential functions signifying the presence of a fast and a slow time constant $\left(\tau_{\text {inaF }}\right.$ and $\left.\tau_{\text {inas }}\right)$. The slow decay constant contributed to about $5 \%$ of the maximal sodium current, while both fast and slow inactivation exhibited striking voltage dependency. The $\tau_{\text {inaF }}$ became faster at more positive potentials (mean $\tau_{\text {inaF }}$ of $889 \mu \mathrm{s}$ at $-47 \mathrm{mV}$ and $410 \mu \mathrm{s}$ at $-2 \mathrm{mV})$. Similarly, $\tau_{\text {inas }}$ also became faster at more positive potentials (mean $\tau_{\text {inas }}$ of $17.4 \mathrm{~ms}$ at $-47 \mathrm{mV}$ and $4.2 \mathrm{~ms}$ at $-2 \mathrm{mV}$ ). Data were plotted against holding voltage and fitted with a single Boltzmann function to calculate mean $\mathrm{V}_{50}(-46.4 \mathrm{mV}$ and $-50.5 \mathrm{mV})$ and slope $(-11.3$ and -7.7) values for the $\tau_{\text {inaF }}$ and $\tau_{\text {inas }}$ components respectively $(n=6)$. g. Representative electrophysiological traces recorded under current clamp in standard aCSF using KGlu filled electrodes before and after perfusion of TTX $(1 \mu \mathrm{M})$. Dotted lines are arranged in $10 \mathrm{mV}$ intervals with top line representing the AP threshold. Note that, TTX caused the development of stable, non-oscillating membrane potential below AP threshold in vlPAG/ DRN DA neurons. $\mathbf{h}$. Representative electrophysiological trace of averaged (150) action potentials and average membrane potential after TTX superfusion $(1 \mu \mathrm{M})$ for the cell shown in $\mathrm{G}$. In this cell the membrane potential was on average $7.2 \mathrm{mV}$ more hyperpolarized compared to the average action potential threshold $(-43.1 \mathrm{mV})$

even at the peak of the AHP with growing contribution during the early phase of the ISI repolarisation. $\mathrm{I}_{\mathrm{NaP}}$ remained inwardly active at the peak of the AHP and activated therafter strongly and quickly compared to $\mathrm{I}_{\mathrm{CaLVA}}$. It displayed similar magnitude to the $\mathrm{I}_{\mathrm{CaLVA}}$ current after $50-75 \mathrm{~ms}$ post $\mathrm{AHP}$ peak but $\mathrm{I}_{\mathrm{NaP}}$ grew rapidly in inward contribution in the latter part of the ISI repolarisation, unlike the $\mathrm{I}_{\mathrm{CaLVA}}$ that maintained a steady (not growing) non-inactivating inward contribution during late ISI repolarization. Collectively, these results highlight the importance of the persistent sodium current activation in driving autorhythmicity in vlPAG/DRN DA neurons and the opposing and facilitating roles of other inward and outward currents in setting the trajectory of voltage during ISI.

\subsubsection{Contribution of individual ionic conductances in depolarization block (DB)}

DA neurons of the vlPAG/DRN, unlike serotonin neurons in the vicinity, exhibit depolarization block (DB), a gradual adaptation and ultimately cessation of firing following challenge with an increasing magnitude depolarizing current pulse (Dougalis et al. 2012). Attainment of a DB state in DA neurons has been postulated to be important in the mode of action 

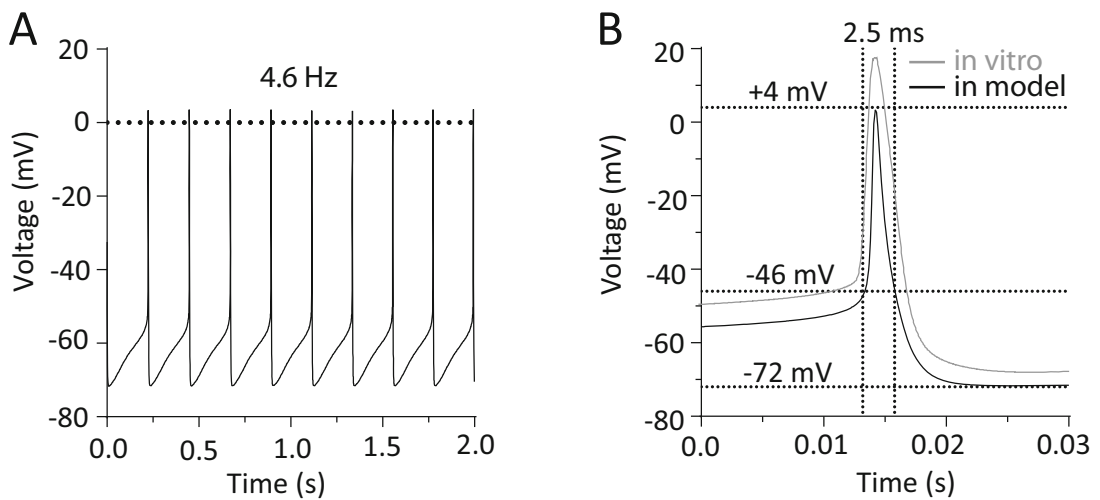

C
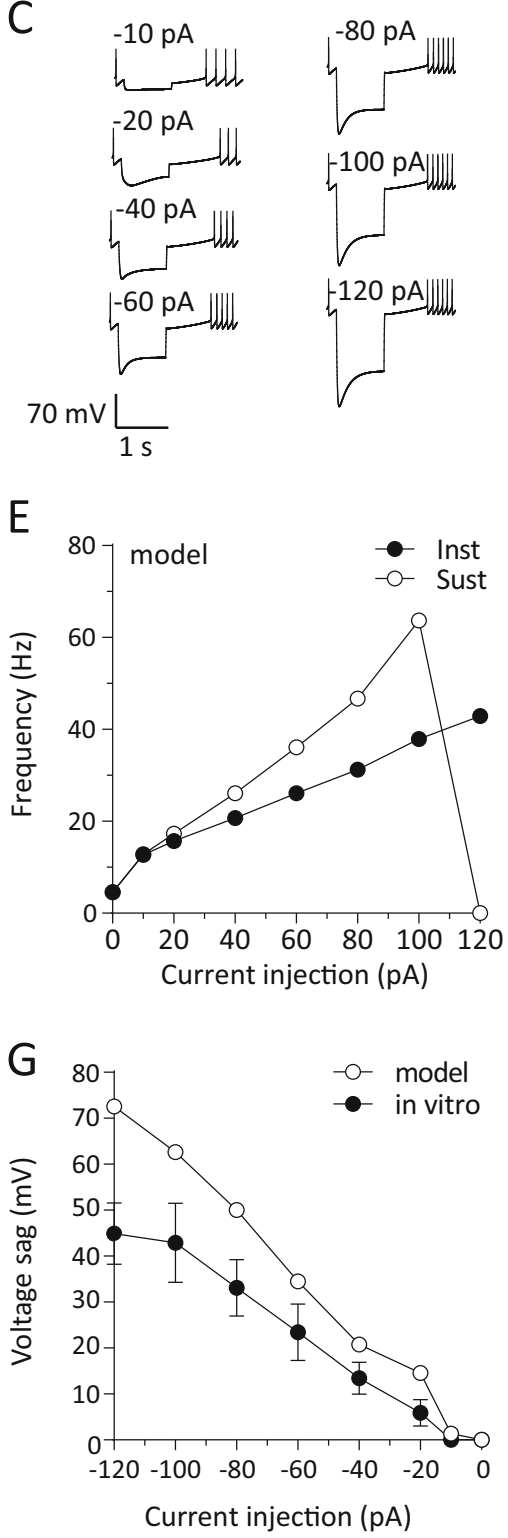

of antipsychotics in schizophrenia (Grace et al. 1997). Our model DA neuron also exhibited DB upon depolarizing current injections at a similar range of depolarizing current
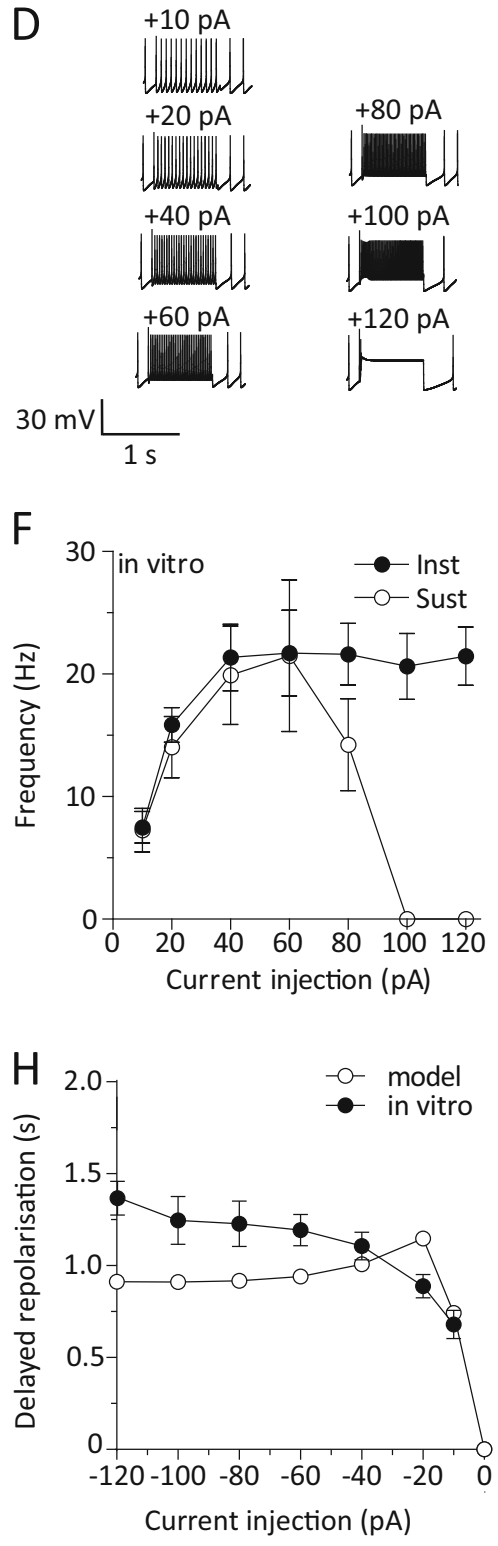

injections as native vlPAG/DRN DA neurons (see Fig. 7d, also Fig. 10a). We thus used the model DA neuron to understand how individual ionic conductances affect the magnitude 
4 Fig. 7 Electrophysiological properties of model DA neuron. a. Representative $2 \mathrm{~s}$ simulation trace of spontaneous AP firing in model DA neuron (model parameters detailed in Table 1). Model DA neuron fired APs at $4.6 \mathrm{~Hz}$. b. Detail of a representative AP in model DA neuron and in vitro DA neuron in vlPAG/DRN. Dotted horizontal lines depict the peak of the $\mathrm{AP}(+4 \mathrm{mV}), \mathrm{AP}$ threshold $(-46 \mathrm{mV})$ and AHP maximal trough $(-72 \mathrm{mV})$. c. Representative simulation traces depicting model DA neuron behaviour following a $1000 \mathrm{~ms}$ incremental hyperpolarizing current injection $(-10$ to $-120 \mathrm{pA})$. Note that, increasing the magnitude of the hyperpolarizing current injection elicited larger voltage-sag responses and delayed repolarisation to firing in model DA neuron. $\mathbf{d}$. Representative simulation traces of AP firing in model DA neuron following $1000 \mathrm{~ms}$ incremental depolarizing current injection ( +10 to $+120 \mathrm{pA})$. Note that, increasing the magnitude of the depolarizing current injection elicited higher frequency of firing and eventually lead to depolarization block (cessation of firing) in model DA neuron. e. Input-output relationship for instantaneous and sustained firing frequency following incremental injection of depolarizing current pulses $(+10$ to $+120 \mathrm{pA})$ in model DA neuron as shown in D. f. Input-output relationship for instantaneous and sustained firing frequency following incremental injection of depolarizing current pulses (+10 to +120 $\mathrm{pA})$ in in vitro vlPAG/DRN DA neurons $(n=5)$. g. Comparative inputoutput relationship for the hyperpolarization-induced voltage-sag in DA model (as shown in C) and in vitro vlPAG/DRN DA neurons $(n=8)$. $\mathbf{h}$. Comparative input-output relationship for delayed repolarisation to firing following the termination of hyperpolarizing current pulses in DA model (as shown in C) and in vitro vlPAG/DRN DA neurons $(n=8)$

of the current required (threshold) to achieved DB by focusing first on sodium and calcium currents. Reducing the $\mathrm{G}_{\max }$ value (to $50 \%$ ) of the $\mathrm{I}_{\mathrm{NaT}}$ resulted in a reduction in the magnitude of the depolarized current required to produce DB (Fig. 10b) while increasing the $\mathrm{G}_{\max }$ (to $150 \%$ ) lead to exactly the opposite effect. In contrast, decreasing $\mathrm{G}_{\max }$ value of the $\mathrm{I}_{\mathrm{NaP}}$ (to $50 \%$ ) resulted in no or a small increase in the DB threshold (Fig. 10c) while increasing $\mathrm{G}_{\max }\left(1.5\right.$ times the $\left.\mathrm{G}_{\max }\right)$ produced a lower threshold for DB. Similar reductions or increases in either $\mathrm{I}_{\mathrm{CaHVA}}$ or $\mathrm{I}_{\mathrm{CaLVA}}$ conductances did not affect the threshold for DB to the extent that sodium conductance modulation revealed in the model DA neuron (Fig. 10d-e) Similarly, moderate changes (reduction/increase) in the $\mathrm{G}_{\max }$ of the $\mathrm{I}_{\mathrm{H}}$ current as imposed previously for the sodium and calcium currents did not produce any significant effects on the threshold of depolarization block (online resource 5A). Changes in the $G_{\max }$ value for the $I_{A}$ potassium current did not have an apparent effect on the DB threshold either (online resource 5B) although further changes beyond 50\% modulated the threshold for DB in a manner similar to the effect of $\mathrm{I}_{\mathrm{NaT}}$ (data not shown). Changes in the $\mathrm{G}_{\max }$ values of the $\mathrm{I}_{\mathrm{M}}$ current caused an effect similar but at much lower magnitude to the $\mathrm{I}_{\mathrm{Kdr}}$ current (online resource $5 \mathrm{C}$ and $\mathrm{D}$ ). The effects of small changes in the $\mathrm{G}_{\max }$ value of the $\mathrm{I}_{\mathrm{Kdr}}$ were more pronounced than the other potassium conductances where $50 \%$ increase or decrease in the $\mathrm{G}_{\max }$ value strongly increased and decreased the DB threshold respectively (online resource 5D). The data suggest that the maximal somatic conductance of transient and persistent sodium currents distinctly modulates, in an opposing manner, the threshold for DB. Similarly, potassium currents reduce the threshold for DB to different degrees but calcium currents do not, indicating the operating complexity between sodium and potassium currents in setting the DB threshold for vlPAG/DRN DA neurons. Imposing larger reductions/increases than $50 \%$ of the maximum conductance of each individual current (e.g. reductions set to less than 20\%) lead to identical changes to those reported above, but also revealed some modulating effects of the $\mathrm{I}_{\mathrm{A}}$ and calcium currents (not seen before at 50 to $150 \%$ level changes imposed), suggesting that under more extreme challenges to the test conditions (longer steps, larger reductions/increases in the maximal conductance) other ionic currents may have a role in setting the DB threshold (data not shown).

\section{Discussion}

DA neurons of the vlPAG and DRN fire action potentials at a frequency of 1-10 Hz but exhibit a much higher coefficient of variation in their firing patterns than SNc DA neurons (Dougalis et al. 2012). Their firing rate and pattern were found to be most similar to those of the VTA DA neurons (Dougalis et al. 2012). However, details of their intrinsic mechanism of operation including, the characteristics of ionic currents and their contribution to spontaneous firing are not known although such mechanisms have been long described for other midbrain DA neuronal groups (see Grace and Onn 1989; Silva et al. 1990; Puopolo et al. 2007; Khaliq and Bean 2010). To address this gap in knowledge we used voltage-clamp electrophysiology and pharmacology to isolate and describe a number of ionic currents operant on adult vlPAG/DRN DA neurons. We then analysed the data using a Hodgkin and Huxley formalism (Hodgkin and Huxley 1952) to construct a conductance-based computer model of a vlPAG/DRN DA neuron. We used this model to perform simulations and

Table 2 A comparison of AP characteristics between in vitro and in silico DA neuron

\begin{tabular}{lll}
\hline Parameter & in vitro DA neuron & model DA neuron \\
\hline Firing frequency, $(\mathrm{Hz})$ & 4.1 & 4.6 \\
AP threshold, $(\mathrm{mV})$ & -43 & -46 \\
AP width at base, $(\mathrm{ms})$ & 4.2 & 2.5 \\
AHP amplitude, $(\mathrm{mV})$ & 25 & 26 \\
AHP maximum, $(\mathrm{mV})$ & -65 & -72 \\
AHP repolarization, $(\mathrm{mV} / \mathrm{ms})$ & 0.12 & 0.08 \\
\hline
\end{tabular}

Comparative summary of characteristics for AP and AHP parameters in computer simulated model DA neuron and native DA neurons recorded in in vitro brain slices (values given as mean, $\mathrm{AP}$ characteristics were measured at AP threshold (width at base) or relative to the AP threshold (AP amplitude, AHP amplitude), while AHP repolarization was measured in the first $100 \mathrm{~ms}$ following the peak of the AHP. AP threshold was determined as the point where the slope of voltage change exceeded $10 \mathrm{mV} / \mathrm{ms}$. All values have been corrected for liquid junction potentials 
A control: $4.6 \mathrm{~Hz}$

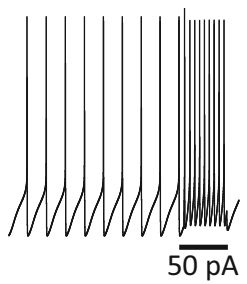

Gmax $0 \%$ CaHVA: $4.5 \mathrm{~Hz}$

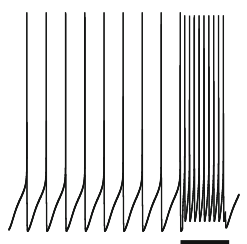

G Gmax $0 \% \mathrm{M}: 9.0 \mathrm{~Hz}$

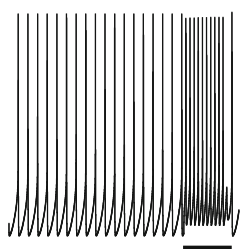

J Gmax $0 \% \mathrm{NaP}: 0 \mathrm{~Hz}$

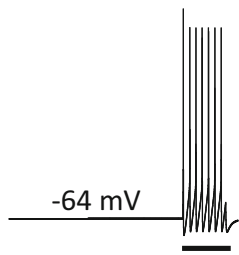

M Gmax $0 \%$ NaT \& $75 \% \mathrm{NaP}: 0 \mathrm{~Hz}$
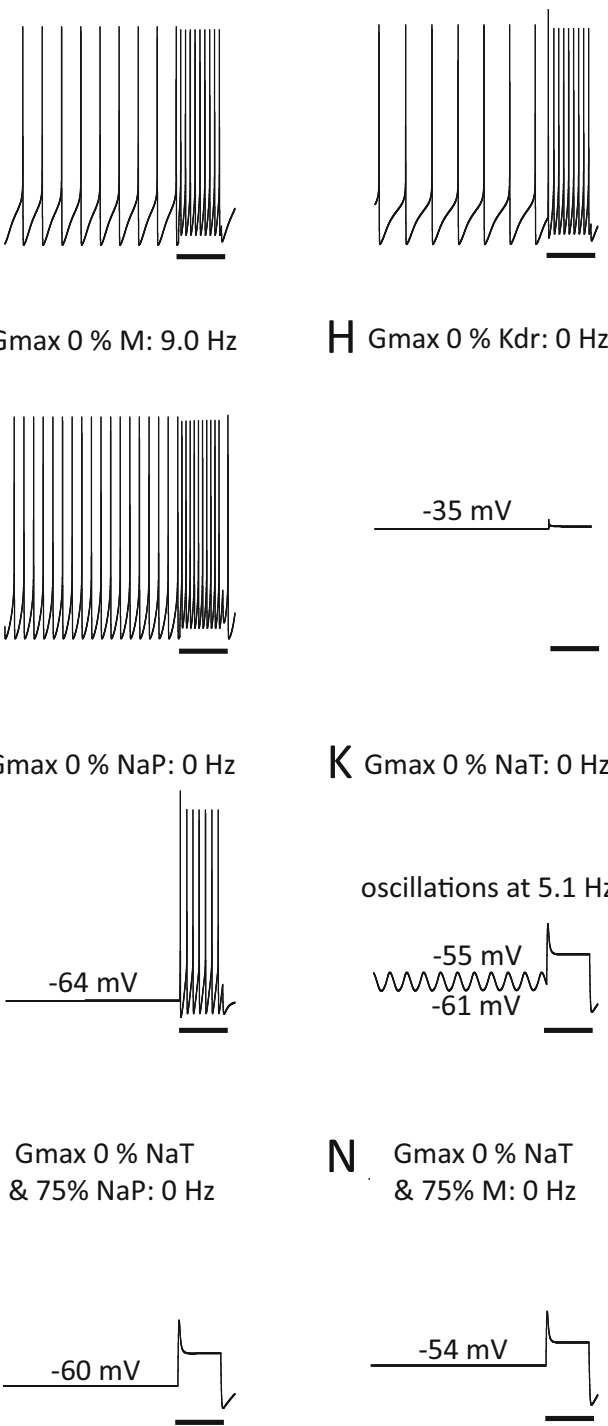

N Gmax $0 \% \mathrm{NaT}$ \& $75 \% \mathrm{M}: 0 \mathrm{~Hz}$
C Gmax 0\% H: $4.3 \mathrm{~Hz}$

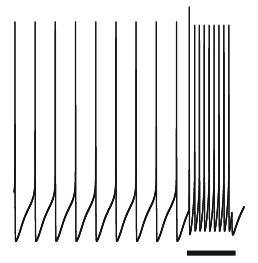

E Gmax $0 \%$ CaLVA: $3.3 \mathrm{~Hz}$

F Gmax 0\% CaHVA

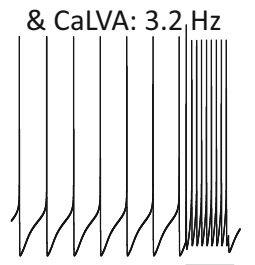

$\mathrm{H}$ Gmax $0 \% \mathrm{Kdr}: 0 \mathrm{~Hz}$

I $\mathrm{Gmax} 0 \% \mathrm{Kdr}$ \& $125 \% \mathrm{M}$ rescue: $0.5 \mathrm{~Hz}$

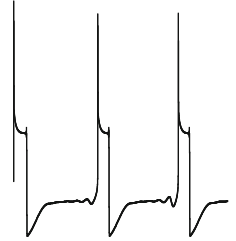

K Gmax $0 \%$ NaT: $0 \mathrm{~Hz}$

oscillations at $5.1 \mathrm{~Hz}$

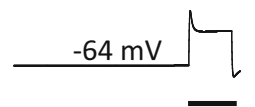

$$
20 \mathrm{mV}
$$

examine the involvement of individual ionic currents in DA neuron's excitability by exploring their contribution in spontaneous firing, pacemaker frequency and threshold for spike frequency adaptation.

DA neurons of the midbrain express a hyperpolarizationactivated cation current $\left(\mathrm{I}_{\mathrm{H}}\right.$ current) that has been long thought to be the hallmark of DA neuron electrophysiology. Recent evidence contest the absolute correlation of an $\mathrm{I}_{\mathrm{H}}$ current with the DA phenotype as some non-DAergic neurons in the VTA also express $I_{H}$ currents (see Margolis et al. 2006) while a subset of VTA DA neurons projecting to the prefrontal cortex (mesocortical projection pathway) do not express an $\mathrm{I}_{\mathrm{H}}$ current (Lammel et al. 2008). In $\mathrm{I}_{\mathrm{H}}$-expressing midbrain DA neurons, blockade of $\mathrm{I}_{\mathrm{H}}$ conductance does not halt autorhythmicity, but instead leads to a reduction in pacemaker firing frequency only in a subtype of SNc, but not in VTA, DA neurons (Neuhoff et al. 2002; Puopolo et al. 2007; Khaliq and Bean 2010). DA vlPAG/DRN neurons expressed an $I_{H}$ current of similar 
4 Fig. 8 Contribution of individual ionic currents to pacemaker firing frequency and autorhythmicity in model DA neuron. Representative simulation traces depicting the contribution of each individual conductance to AP firing frequency in model DA neuron. Maximal conductance of each current was reduced to $0 \%$ of its maximal conductance value $\left(\mathrm{G}_{\max }\right)$ and the effects on AP firing frequency were noted. The bar appearing at the end of each trace is the response to a 50 pA depolarising current injection $(500 \mathrm{~ms})$. a: The model DA neuron fired APs at a basal firing frequency of $4.6 \mathrm{~Hz}$. b: $\mathrm{I}_{\mathrm{A}}$ current elimination lead to a two-fold increase in firing frequency of model DA neuron. $\mathbf{c}$ : Elimination of the $\mathrm{I}_{\mathrm{H}}$ current induced a small decrease in firing frequency of model DA neuron. $\mathbf{d}$ : Elimination of the $\mathrm{I}_{\mathrm{CaHVA}}$ did not modify firing frequency of model DA neuron. e: Elimination of the $\mathrm{I}_{\mathrm{CaLVA}}$ reduced the frequency of firing of model DA neuron by $25 \%$. f: Concomitant elimination of both $\mathrm{I}_{\mathrm{CaHVA}}$ and $\mathrm{I}_{\mathrm{CaLVA}}$ calcium conductances did not eliminate spontaneous firing of model DA neuron. $g$ : $\mathrm{I}_{\mathrm{M}}$ current elimination lead to a two-fold increase in firing frequency of model DA neuron. $\mathbf{h}$ : Elimination of $\mathrm{I}_{\mathrm{Kdr}}$ current stopped spontaneous firing of model DA neuron. $i$ : A small increase of $I_{M}$ current (to $125 \%$ of basal $G_{\max }$ ) in the absence of a $\mathrm{I}_{\mathrm{Kdr}}$ current restored spontaneous firing of model DA neuron. j: Elimination of $\mathrm{I}_{\mathrm{NaP}}$ current lead to the development of a stable nonoscillating membrane potential below AP threshold. However, upon depolarising current injection the model DA neuron still fired APs. k: Elimination of $\mathrm{I}_{\mathrm{NaT}}$ current lead to the development of an oscillating membrane potential below AP threshold (oscillating range, max, $-55 \mathrm{mV}$; min, $-61 \mathrm{mV}$ ). Upon depolarising current injection the model DA neuron did not fire APs. I: Concomitant elimination of both $\mathrm{I}_{\mathrm{NaT}}$ and $\mathrm{I}_{\mathrm{NaP}}$ sodium conductances lead to the development of a stable nonoscillating membrane potential $(-64 \mathrm{mV})$ below AP threshold and cessation of spontaneous AP firing. Upon depolarising current injection the model DA neuron did not fire APs. m: A small decrease (to $75 \%$ of $\mathrm{G}_{\max }$ ) in $\mathrm{I}_{\mathrm{NaP}}$ in the absence of $\mathrm{I}_{\mathrm{NaT}}$ current stopped the development of a background oscillation in model DA neuron. $\mathbf{n}$ : A small decrease (to $75 \%$ of $\mathrm{G}_{\max }$ ) in $\mathrm{I}_{\mathrm{M}}$ current in the absence of a $\mathrm{I}_{\mathrm{NaT}}$ current was also successful (as was $I_{\mathrm{NaP}}$ alone, see above $\mathrm{M}$ ) to stop the development of a background oscillation in model DA neuron

magnitude and kinetics with that of VTA, but not of that of the SNc, DA neurons (online resource 1). Blockade of $\mathrm{I}_{\mathrm{H}}$ current in DA vlPAG/DRN neurons resulted, as expected, in ablation of the hyperpolarization-induced, slowly activating, inward current (under voltage-clamp) or the depolarizing voltage-sag (under current-clamp) but did not significantly affect basal firing frequency or pacemaker variability in vitro. The DA model and in vitro brain slice data were in good agreement regarding the magnitude and kinetics of the $\mathrm{I}_{\mathrm{H}}$ current-mediated voltagesag. In our DA model, reduction or complete removal of the $\mathrm{I}_{\mathrm{H}}$ current did not halt autorhythmicity and did not have large effects on firing of the model DA neuron, although it resulted in a reduction in basal firing frequency (about 10\%). This is largely because the $V_{50}$ for activation of the $I_{H}$ current $(-115 \mathrm{mV})$ is more negative than the subthreshold potential that the neuron usually attains in vitro (normally up to $-70 \mathrm{mV}$ ) and thus the $\mathrm{I}_{\mathrm{H}}$ current does not get efficiently activated during AHP following an AP (estimated availability at $-70 \mathrm{mV}$ of less than $5 \%$ ). The more positive $\mathrm{V}_{50}$ for $\mathrm{I}_{\mathrm{H}}$ current activation of the SNc DA neurons $(25 \mathrm{mV}$ more positive than vlPAG/DRN or VTA DA neurons, see online resource 1) could potentially explain the effects of $\mathrm{I}_{\mathrm{H}}$ current blockade on $\mathrm{SNc}$ DA neuron's firing rate and the lack of effects in VTA DA neurons firing rate reported previously (Neuhoff et al. 2002; Puopolo et al. 2007, Khaliq and Bean 2010) or the lack of effects seen here for vlPAG/DRN DA neurons. Examination of the trajectory of activation of $\mathrm{I}_{\mathrm{H}}$ current in the model DA neuron during the ISI suggests that a small $\mathrm{I}_{\mathrm{H}}$ inward current declines steadily from the peak of AHP to AP threshold giving rise to a net outward current during ISI repolarization. The $\mathrm{I}_{\mathrm{H}}$ current did not modulate the threshold for DB (online resource 5) further arguing that this current plays no significant role in vlPAG/DRN DA neuron excitability. These data taken together suggest that, similar to VTA DA neurons (Khaliq and Bean 2010), autorhythmicity of adult vlPAG/ DRN DA neurons is largely independent of the $\mathrm{I}_{\mathrm{H}}$ current which contributes little in setting their basal firing frequency or influencing neuronal excitability.

Repolarization of DA neurons during the ISI and tuning of pacemaker frequency in individual SNc and VTA neurons is dependent on expression of an A-type transient potassium current $\left(I_{A}\right.$ current, Silva et al. 1990; Liss et al. 2001; Koyama and Appel 2006a). The diversity of $\mathrm{I}_{\mathrm{A}}$ current behavior is dependent on the different number of potassium transcripts that can contribute to the assembly of these channels. Kv4.1, Kv4.2, Kv4.3, $\mathrm{Kv} 3.4$ and Kv1.4 subunits have been reputed to give rise to transient $\mathrm{I}_{\mathrm{A}}$-like currents but only the Kv4.3 transcripts were found expressed in SNc DA neurons (Liss et al. 2001). SNc DA neuron's $\mathrm{I}_{\mathrm{A}}$ currents are mediated by the Kv4.3 (long) transcript (heteropoda toxin 3-sensitive) where it seems to form functional $\mathrm{I}_{\mathrm{A}}$ channels in combination with accessory protein KChip3 (but not KChip 1,2 or 4, see Liss et al. 2001). Using a two protocol subtraction method (as reported before, Koyama and Appel 2006a), we recorded outward transient currents sensitive to 4-AP blockade which possessed a voltage-dependent activation time constant consistent with previous reports (Liss et al. 2001; Koyama and Appel 2006a). However, in our study the activation $V_{50}$ for the $I_{A}$ current was found to be 30 and $20 \mathrm{mV}$ more negative to what has been reported previously for $\mathrm{SNc}$ and VTA DA neurons respectively (Liss et al. 2001; Koyama and Appel 2006a), while the inactivation $V_{50}$ was similar to the study of VTA DA neurons (approximately $-90 \mathrm{mV}$, Koyama and Appel 2006a) and about $30 \mathrm{mV}$ more negative to what has been reported in SNc DA neurons recorded under whole-cell conditions (Liss et al. 2001). Additionally, inactivation of the $\mathrm{I}_{\mathrm{A}}$ currents in vlPAG/DRN DA neurons studied here was described better with the sum of two exponentials at potentials more positive than $-30 \mathrm{mV}$, a fast (about $100 \mathrm{~ms}$, voltagedependent) and a slow (about $550 \mathrm{~ms}$, voltage-independent), which differs kinetically from the reported monoexponential and voltage-independent fast decay (around 30-50 ms) of $\mathrm{I}_{\mathrm{A}}$ currents in both SNc and VTA neurons in vitro (Silva et al. 1990; Liss et al. 2001; Koyama and Appel 2006a). The differences in the activation /inactivation range and kinetics of the inactivation of $\mathrm{I}_{\mathrm{A}}$ currents described here with previous reports of $\mathrm{I}_{\mathrm{A}}$ currents in VTA and SNc DA neurons can be attributed to 

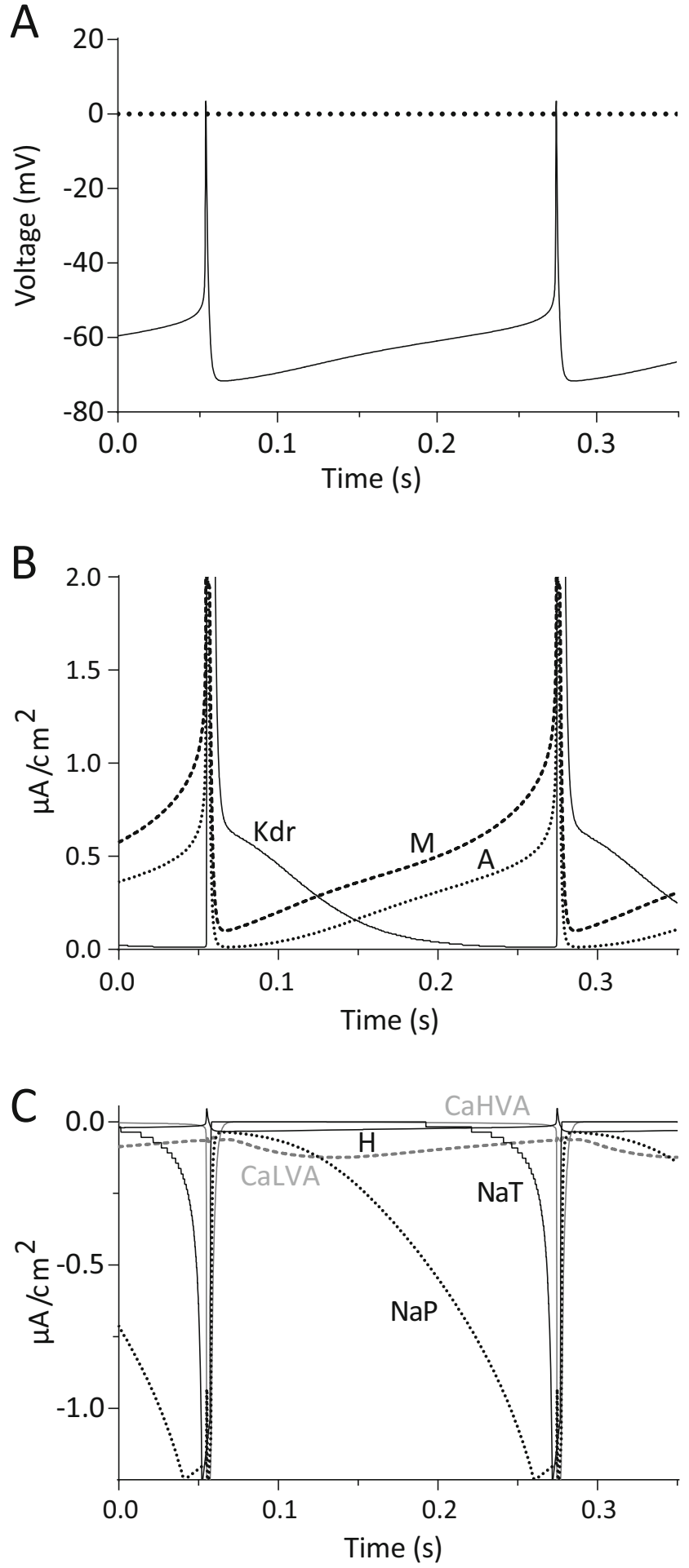

differences in the preparations (e.g. physiological versus room temperature, brain slices versus dissociated neurons, whole-cell recordings versus outside-out patches) or inherently differential calcium influx kinetics (that may affect the properties of $\mathrm{I}_{\mathrm{A}}$ currents recorded, see Koyama and Appel 2006a)and residual recruited currents. By exclusion of the above possibilities, the discrepancies can also represent a real difference in the gating properties of $\mathrm{I}_{\mathrm{A}}$ currents in vlPAG/DRN DA neurons given that
Fig. 9 Contribution of individual ionic currents to interspike interval (ISI) repolarisation in model DA neuron. a: Simulation trace depicting the voltage trajectory during ISI between two successive APs in model DA neuron. b: Simulation trace depicting the current flowing through three individual outward potassium currents $\left(\mathrm{A}, \mathrm{M}\right.$ and $\left.\mathrm{K}_{\mathrm{dr}}\right)$ during the ISI of two successive APs (voltage response as shown in A). Note that, the deactivation of the $\mathrm{IK}_{\mathrm{dr}}$ facilitates repolarisation from AHP peak to AP threshold while the activation of the $\mathrm{I}_{\mathrm{M}}$ and $\mathrm{I}_{\mathrm{A}}$ potassium currents oppose repolarisation. c: Representative simulation traces depicting the kinetics of inward sodium, calcium and H-type currents during the ISI of two successive APs (voltage response as shown in A). Note that, a persistent sodium current $\left(\mathrm{I}_{\mathrm{NaP}}\right)$ activated shortly after the AHP peak giving rise to a strong inward current. Similarly, but displaying very different kinetics in activation and magnitude of response, $\mathrm{I}_{\mathrm{CaLVA}}$ gave rise to a small but non-inactivating inward current during ISI. In contrast, $\mathrm{I}_{\mathrm{CaHVA}}$ current did not activate at all during ISI, while the small magnitude activation of the $\mathrm{I}_{\mathrm{H}}$ current (a declining inward conductance) gave rise to a net outward current during ISI.

SNc and VTA DA neurons exhibit projection-specific differential physiological characteristics and greater diversity in their properties than previously envisaged (e.g see results from Lammel et al. 2008, 2011). The methodological differences between our study and the available studies (Liss et al. 2001; Koyama and Appel 2006a) could explain some of the differences observed in $\mathrm{I}_{\mathrm{A}}$ current gating in vlPAG/DRN DA neurons. For example, $\mathrm{I}_{\mathrm{A}}$ channels may be distributed both in somatic and dendritic domains but the latter are severed in dissociated neurons while often a mixture of somatic/extrasomatic channels is sampled in outside-out patch recordings leading to potential difference in the kinetics recorded (as acknowledged by Liss and colleagues, a $15 \mathrm{mV}$ difference in inactivation $V_{50}$ value was seen between whole-cell and outside-out patch recordings in SNc DA neurons). Also, in both the studies of Liss and colleagues and Koyama and Appel, only a single exponential was fitted and reported for $\mathrm{I}_{\mathrm{A}}$ currents despite the fact that the currents could have had more complex inactivation properties (referred to as dominant fast time constant for SNc DA neurons in Liss et al. 2001; see also clear evidence of biexponential decay in $\mathrm{I}_{\mathrm{A}}$ current recordings from dissociated VTA DA neurons in Koyama and Appel 2006a, figure 1A2). In our hands, VTA and vlPAG/DRN DA neurons exhibited similar $\mathrm{I}_{\mathrm{A}}$ current behavior with slow (100-150 ms inactivation time constant), initially monoexponential decay (at more negative potentials than $-40 \mathrm{mV}$ ) with biexponential inactivation apparent at more positive potentials than $-30 \mathrm{mV}$ on long steps (see tail currents in online resource 1). The available data derived from the interpretation of the three main studies of $\mathrm{I}_{\mathrm{A}}$ current kinetics (Silva et al. 1990; Liss et al. 2001 and Koyama and Appel 2006a) and the evidence from projection specific studies of DAergic populations (see Lammel et al. 2008, 2011) suggest that $I_{A}$ current properties in SNc and VTA DA neurons are not similar but a systematic study of the $\mathrm{I}_{\mathrm{A}}$ current properties of projection-specific DA groups is still lacking making further interpretation and comparison to our data difficult. Interestingly, in our hands, 4-AP sensitive 
Fig. 10 Contribution of individual ionic currents to depolarization block (DB) in model DA neuron. a.

Representative $5 \mathrm{~s}$ simulation traces depicting the responses of model DA neurons to a sequence of depolarizing current injections (500 ms, black lines) leading to DB under basal control conditions. b. Strong effects of increasing (left, $150 \%$ of $\mathrm{G}_{\max }$ ) and decreasing (right, 50\% of $\mathrm{G}_{\mathrm{max}}$ ) of $\mathrm{I}_{\mathrm{NaT}}$ on the threshold of DB. c. Strong effects of increasing (left, $150 \%$ of $\mathrm{G}_{\max }$ ) and decreasing (right, $50 \%$ of $\mathrm{G}_{\max }$ ) of $\mathrm{I}_{\mathrm{NaP}}$ on the threshold of DB. The effects of manipulating $\mathrm{I}_{\mathrm{NaP}}$ on DB threshold were in opposite polarity from those observed by manipulation of $\mathrm{I}_{\mathrm{NaT}}$ (see $\mathrm{B}$ above). d. No apparent effects of increasing (left, $150 \%$ of $\mathrm{G}_{\max }$ ) or decreasing (right, $50 \%$ of $\mathrm{G}_{\max }$ ) of $\mathrm{I}_{\mathrm{CaHVA}}$ on the threshold of DB. e. No apparent effects of increasing (left, $150 \%$ of $\mathrm{G}_{\max }$ ) or decreasing (right, $50 \%$ of $\mathrm{G}_{\max }$ ) of $\mathrm{I}_{\mathrm{CaLVA}}$ on the threshold of DB

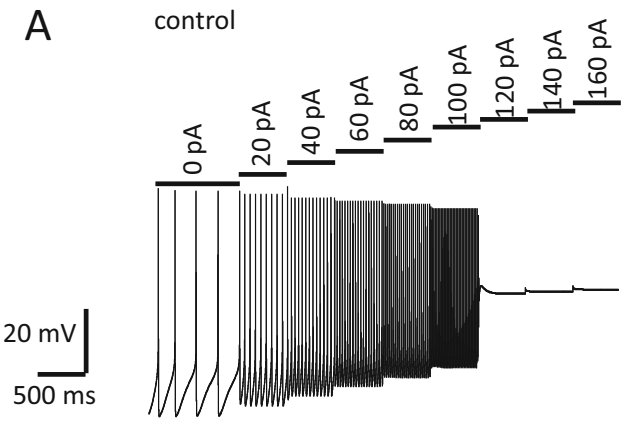

B

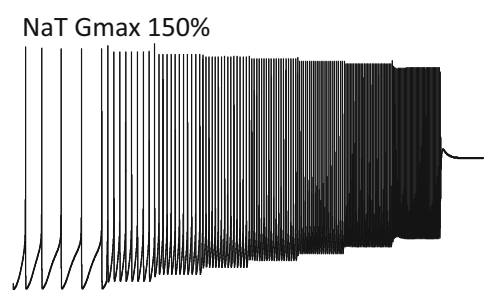

NaT Gmax 50\%

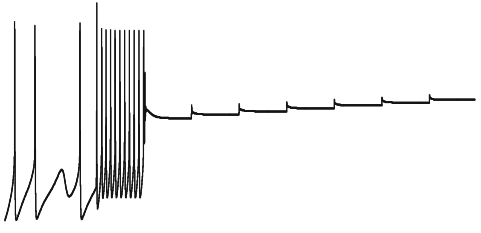

C
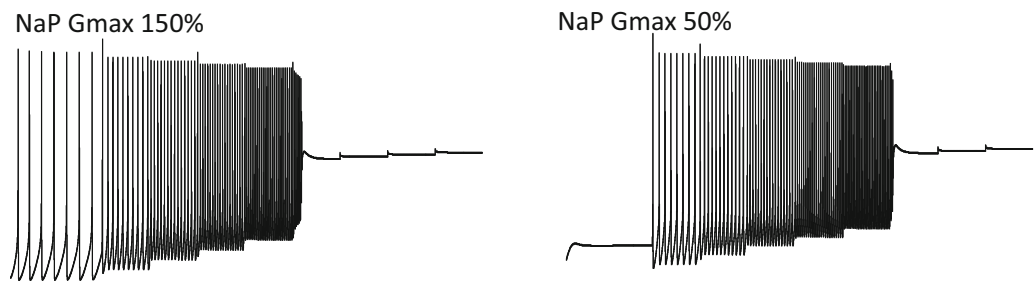

D

CaHVA Gmax $150 \%$
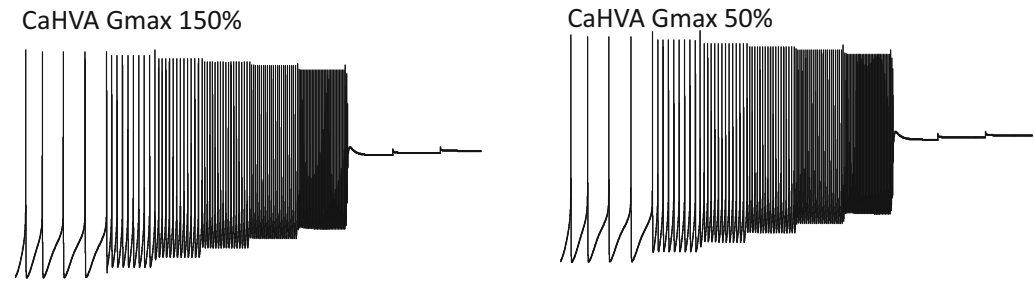

E CaLVA Gmax $150 \%$

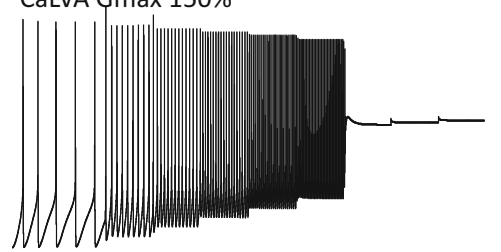

CaLVA Gmax 50\%

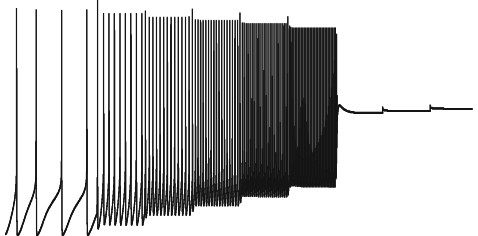

currents also exhibited a bi-exponential inactivation (online resource 2) in a similar manner to those recorded with the two protocol subtraction method. In our experiments, 4-AP increased a background conductance leading to typical current 'crossing' upon current subtraction in good agreement to what has been reported before by others in DA neurons (see Liss et al. 2001; Khaliq and Bean 2008) indicating some of the potential inherent difficulties in using 4-AP as a tool to kinetically describe $\mathrm{I}_{\mathrm{A}}$ currents. Despite this, a third method of isolating the $\mathrm{I}_{\mathrm{A}}$ conductance (recordings in the presence of TEA as in Silva et al. 1990) also showed that the $I_{A}$ currents in vlPAG/
DRN DA neurons exhibit a strong biexponential decay with the fast exponential decay being twice as long (approximately $70 \mathrm{~ms}$, albeit voltage-independent) compared to the reports of Liss and colleagues or Koyama and Appel suggesting that the biexponential properties seen here should most likely reflect $\mathrm{I}_{\mathrm{A}}$ current physiological operation in adult vlPAG/DRN DA neurons in brain slices. Also, all three methods of $\mathrm{I}_{\mathrm{A}}$ current isolation used here resulted in commensurate $V_{50}$ values for the activation of the current (around -55 to $-60 \mathrm{mV}$ ) suggesting further that the kinetics of activation described here are likely to reflect $\mathrm{I}_{\mathrm{A}}$ current gating in situ in brain slices rather than an 
artefact of the isolation method. The contribution of other background residual currents that could potentially contaminate our $\mathrm{I}_{\mathrm{A}}$ recording (e.g. a calcium-activated potassium conductance, $\mathrm{I}_{\mathrm{H}}$ current) cannot be excluded, although such a proposal could not directly explain the biexponential decay of the 4-APsensitive currents seen here or the biexponential slow decay seen in TEA recordings. Using our voltage-clamp data, we modelled a reduced version of $\mathrm{I}_{\mathrm{A}}$ current with a single, voltage-dependent exponential decay according to the two protocol subtraction method. Our model DA neuron exhibited well-defined delayed repolarization upon hyperpolarizing current steps to $>-80 \mathrm{mV}$ under current-clamp that was carried forward by the $\mathrm{I}_{\mathrm{A}}$ conductance and was quantitatively similar to delayed repolarization to firing recorded in brain slices in vitro. The model DA neuron predicted that a reduction in $\mathrm{I}_{\mathrm{A}}$ currents will lead to an increase in pacemaker firing frequency an effect seen previously in SNc DA neurons using blockade with heteropoda toxin-3 (Liss et al. 2001). $\mathrm{I}_{\mathrm{A}}$ currents develop a strong outward current during ISI repolarization from the peak of the AHP to AP threshold. Although abolition of the $\mathrm{I}_{\mathrm{A}}$ current lead to a two-fold increase in firing frequency, it did not stop spontaneous firing, suggesting that $\mathrm{I}_{\mathrm{A}}$ current is not essential for autorhythmicity. Interestingly, blocking the $\mathrm{I}_{\mathrm{A}}$ current in the DA neuron model did not strongly reduce the threshold for DB (online resource 5) suggesting that $\mathrm{I}_{\mathrm{A}}$ currents are major determinants of neuronal excitability in DA vlPAG/DRN neurons but do not exhibit high propensity in modulating $\mathrm{BD}$ threshold at least when exposed to the sequences and range of depolarizing pulses studied herein. Indeed, longer pulses (> $1000 \mathrm{~ms}$ ) or greater changes in $\mathrm{G}_{\max }$ rendered the DA model neuron more susceptible to the effects of the $\mathrm{I}_{\mathrm{A}}$ current (data not shown) suggesting that under certain, more robust, conditions DB threshold is also modulated by $\mathrm{I}_{\mathrm{A}}$ currents. In a similar manner to $I_{A}$ potassium currents, blockade of $I_{K d r}$ positively modulated firing frequency in the DA neuron model but also modulated strongly the threshold for DB (online resource 5). The deactivation of $\mathrm{I}_{\mathrm{Kdr}}$ following the AHP had a dramatic effect in membrane repolarization during ISI repolarization to AP threshold. We also show here that outward potassium currents recorded in vlPAG/DRN DA neurons are a composite of different non-inactivating/slowly-inactivating currents including a non-inactivating potassium conductance activating at more negative potentials than typical $\mathrm{I}_{\mathrm{Kdr}}$ currents and mediated through XE991-sensitive KCNQ (Kv7) channels suggesting that an M-current $\left(\mathrm{I}_{\mathrm{M}}\right)$ is also expressed in DA vlPAG/DRN neurons with similar properties to that expressed in VTA DA neurons (online resource 3, see also Koyama and Appel 2006b, see also Hansen et al. 2006; Drion et al. 2010). The complete abolition of $\mathrm{I}_{\mathrm{Kdr}}$ current renders the neuron highly depolarized and stops autorhythmicity. This is largely because $\mathrm{I}_{\mathrm{Kdr}}$ current is the only persistent outward current in this neuron that can help facilitate effective repolarization after an AP by virtue of its deactivation. Despite the three-fold lower maximal conductance of the $\mathrm{I}_{\mathrm{M}}$ current compared to the $\mathrm{I}_{\mathrm{Kdr}}$ current used in our model, increasing the $\mathrm{I}_{\mathrm{M}}$ current contribution by just minute amounts $(10-25 \%)$ rescues the spontaneous firing of the DA neuron in the complete absence of an $\mathrm{I}_{\mathrm{Kdr}}$ current, leading to the establishment of low frequency rhythmic APs and plateaus in the DA model neuron. The model suggests that $I_{M}$ current does not inactivate completely following AHP, thus providing a steady background outward current even at the peak of the AHP. This explains the fact that increase of $\mathrm{I}_{M}$ current rescues neuron's spontaneous firing in the absence of $\mathrm{I}_{\mathrm{Kdr}}$ while abolition of the $\mathrm{I}_{\mathrm{M}}$ current dramatically increased firing frequency (and induced a depolarising current in vitro) but did not halt spontaneous firing of the DA model neuron (as partially seen in Koyama and Appel 2006b also see opposite effects of $I_{M}$ current opener retigabine in Hansen et al. 2006). The $I_{M}$ current also had an effect on the threshold for DB in a manner similar, but far less stronger to the $\mathrm{I}_{\mathrm{Kdr}}$ current (see online resource 5). Our model suggests that the $\mathrm{I}_{\mathrm{M}}$ current is efficiently activated during ISI repolarization and in tandem with the $\mathrm{I}_{\mathrm{A}}$ current provides a strong outward current delaying the repolarization to AP threshold. These results collectively suggest that potassium currents strongly modulate neuronal excitability of vlPAG/DRN neurons but are not necessary individually per se for the expression of autorhythmicity. It is rather that, the complete negation of sustained outward currents like $\mathrm{I}_{\mathrm{Kdr}}$ and $\mathrm{I}_{\mathrm{M}}$ simultaneously, is the prerequisite to render the neuron inactive and unable to generate rhythmically APs in line with a Hodgkin and Huxley formalism (Hodgkin and Huxley 1952).

Calcium currents play an important role in the pacemaking process in a number of diverse neuronal preparations, from midbrain SNc DA neurons to hypothalamic GABAergic suprachiasmatic nucleus neurons (e.g. Jackson et al. 2004; Khaliq and Bean 2010). Unlike SNc DA neurons, VTA DA neurons express a sodium 'persistent' background current that dominates over the rather small calcium current in the subthreshold range during ISI (Puopolo et al. 2007; Khaliq and Bean 2010). In our study, similar to the results obtained by Khaliq and Bean (2010) in VTA DA neurons, blockade of voltage-gated calcium currents by low calcium/high magnesium solutions lead to an increase in the pacemaker frequency in vlPAG/DRN DA neurons. Although such a treatment could have also reduced calcium-dependent potassium conductances leading to an increase in firing rate (but see also studies of Wolfart and Roeper 2002; Deignan et al. 2012), it demonstrates that pacemaking can persist in the absence of calcium indicating that calcium is not critical in driving spontaneous firing of vlPAG/DRN DA neurons. Interestingly, removal of calcium from the extracellular solution lead to small, albeit statistically insignificant reduction in the pacemaker variability unlike what would be expected from blockade of calcium-dependent potassium currents (see Wolfart et al. 2001), implying that this change represents potentially the algebraic sum of negating all calcium-driven and calcium-dependent currents (with potentially opposing roles in 
pacemaker variability, see Poetschke et al. 2015) in vlPAG/ DRN DA neurons. Our experiments indicate that both transient LVA and slowly-inactivating HVA (through a calcium releasemediated calcium inactivation) calcium currents are expressed in vlPAG/DRN DA neurons with properties similar to those described previously in other systems (Brevi et al. 2001; Jackson et al. 2004) but also in DA neurons (Kang and Kitai 1993a, 1993b). The slowly inactivating HVA component was sensitive to nimodipine, indicating that an L-type calcium current is partly responsible for the inward conductance recorded (online resource 4). The LVA component had characteristics reminiscent of a T-type current (see Kang and Kitai 1993a), was resistant to nimodipine (online resource 4) but sensitive to cadmium. Our model suggests that full blockade of the $\mathrm{I}_{\text {CaLVA }}$ currents, did not halt DA model neuron autorhythmicity or affect its threshold for DB but reduced basal pacemaker firing frequency by nearly $30 \%$. $\mathrm{I}_{\mathrm{CaLVA}}$ currents contributed with a small (compared to potassium currents) but constant inward current (that did not fully inactivate during or shortly after AP) during ISI repolarization to AP threshold. In contrast, to the $\mathrm{I}_{\mathrm{CaLVA}}$ currents, $\mathrm{I}_{\mathrm{CaHVA}}$ currents failed to contribute with any inward current during ISI repolarization to AP threshold, remaining essentially inactive during ISI and activating only during the AP. Blocking $\mathrm{I}_{\mathrm{CaHVA}}$ current did not halt autorhythmicity of DA model neuron (alone or in combination with blockade of the $\mathrm{I}_{\mathrm{CaLVA}}$ currents) and did not affect pacemaker firing frequency or contribute to any degree in setting the threshold for DB (even on longer steps as described for the $\mathrm{I}_{\mathrm{A}}$ current). This is perhaps expected by $\mathrm{I}_{\mathrm{CaHVA}}$ current that has on average nearly $30 \mathrm{mV}$ more positive activation midpoint in comparison to the $\mathrm{I}_{\mathrm{CaLVA}}$ current, explaining why the latter can affect neuronal operation in the subthreshold range. Our data collectively suggest that calcium currents are not required for spontaneous firing on the model DA neuron but $\mathrm{I}_{\mathrm{CaLVA}}$ currents may have a role in modulating neuronal excitability in the opposite direction and less strongly than potassium conductances.

Sodium currents play an important role in the pacemaking activity of VTA DA neurons driving repolarization during ISI through a voltage-dependent, TTX-sensitive and a voltage-independent, TTX-resistant component (see Khaliq and Bean 2010). They are less important in the pacemaking of SNc DA neurons where calcium currents dominate and drive repolarization during the ISI (Puopolo et al. 2007). Blocking TTXsensitive sodium channels in the brain slice lead to a stable, non-oscillating, resting membrane potential in vlPAG/DRN DA neurons, in a similar manner to what has been reported previously for VTA DA neurons (Khaliq and Bean 2010) and in contrast to the reported behavior of SNc DA neurons (see Nedergaard et al. 1993; Mercuri et al. 1994; Chan et al. 2007; Puopolo et al. 2007; Guzman et al. 2009). In the model DA neuron, blockade of both fast and persistent sodium conductances lead to the establishment of a hyperpolarized stable non- oscillating membrane potential in the same manner as in the in vitro brain slice. $\mathrm{I}_{\mathrm{NaT}}$ and $\mathrm{I}_{\mathrm{NaP}}$ currents start activating at different time points and rates following AHP during ISI repolarization and are adominant force driving DA neurons towards AP threshold. $\mathrm{I}_{\mathrm{NaP}}$ current does not fully inactivate following an $\mathrm{AP}$ and rapidly activate during ISI repolarization relatively fast when compared to $\mathrm{I}_{\mathrm{NaT}}$ current that is engaged significantly only in the last $50 \mathrm{~ms}$ before the occurrence of a AP under normal conditions. Blockade of $\mathrm{I}_{\mathrm{NaP}}$ current alone halted spontaneous firing but depolarizing current injections restored the firing capacity of the DA model neuron suggesting that the expression of spontaneous firing is affected by the $\mathrm{I}_{\mathrm{NaP}}$ current but not the capacity to generate APs in response to external inputs. Blockade of $\mathrm{I}_{\mathrm{NaT}}$ currents alone stopped all spiking behavior on model DA neuron, inclusive of depolarizationinduced spiking, and lead to a relatively hyperpolarized (compared to AP threshold) membrane potential with evidence of background oscillations (at $5 \mathrm{~Hz}$ ) that were dependent on an interaction of the $\mathrm{I}_{\mathrm{NaP}}$ current and the $\mathrm{I}_{\mathrm{M}}$ current. Indeed, small reductions (i.e. 10-25\%) in either $\mathrm{I}_{\mathrm{M}}$ current or $\mathrm{I}_{\mathrm{NaP}}$ current during the absence of a $\mathrm{I}_{\mathrm{NaT}}$ current obliterated the oscillatory background behavior and resulted in a steady-state non-oscillating hyperpolarizing potential without affecting the overall working of the model. Such a behavior was not seen in the brain slice preparation suggesting that it could be related to the direct interaction of the activation kinetics of outward and inward persistent conductances in the model but its physiological significance (if any) is not currently known. Perhaps, studies utilizing dynamic voltage-clamp techniques to selectively negate $\mathrm{I}_{\mathrm{NaP}}$ or $\mathrm{I}_{\mathrm{NaT}}$ conductance in conjunction with pharmacology could help enormously in clarifying the distinct roles of transient and persistent sodium currents (or any interactions of the latter with the $\mathrm{I}_{\mathrm{M}}$ seen in the model DA neuron) in setting the properties of in vitro adult vlPAG/DRN DA neurons. Finally, using the DA model neuron we found that both transient and persistent sodium currents affected strongly DB threshold albeit exhibiting opposing effects. In good agreement with our study, Tucker et al. (2012) also found that reduction in somatic transient sodium currents reduces the DB threshold in a both in vitro and model SNc DA neuron (Tucker et al. 2012). During our voltage-clamp analysis, we could not reliably detect any discernable sodium current that persisted in the presence of TTX and thus we infer that TTX-resistant currents are not operant on vlPAG/DRN DA neurons, although it is also possible that we may have missed this small component. Our data collectively suggest that sodium currents endow DA vlPAG/DRN neurons with autorhythmicity by slowly activating during ISI repolarization and effectively bringing the neuron towards the AP threshold for firing.

In conclusion, the data presented here extend our previous physiological characterization (Dougalis et al. 2012) and argue that DA neurons of the vlPAG/DRN express autorhythmicity in the absence of synaptic transmission via the interplay of 
potassium and sodium currents without the absolute need of calcium currents. The properties of the ionic currents recorded here $\left(\mathrm{I}_{\mathrm{H}}\right.$ current, $\mathrm{I}_{\mathrm{A}}$ current), the lack of small oscillating potentials in the presence of sodium channel blockers taken together with the mechanisms for autorhythmicity (reliance more on sodium rather than calcium currents) also support further the idea that vlPAG/DRN DA neurons are operationally similar to VTA, rather than SNc, DA neurons. In particular, the properties of a slowly inactivating $\mathrm{I}_{\mathrm{A}}$ current in conjunction with the small and slowly activating $\mathrm{I}_{\mathrm{H}}$ current described herein pinpoint that vlPAG/DRN DA neurons are most similar to prefrontal cortex or medial shell of nucleus accumbens projecting DA neurons (see Lammel et al. 2008, 2011). Given the importance of vlPAG/DRN DA neurons in arousal, reward and nociception, it would be interesting in the future to understand how the electrical properties and the synaptic activity arriving at these neurons may be modulated by sleep-wake cycles, drugs of abuse (e.g cocaine, heroin), or how it may change in models of nociception (e.g. following spinal cord injury or in neuropathic pain).

Acknowledgements This research was supported by grant U120085816 from the U.K. Medical Research Council (MRC) and a University Research Fellowship from The Royal Society to M.A.U. and, by the German DFG project LI1745/1 to B.L.

Compliance with ethical standards All animal breeding and experimental procedures were conducted in accordance with the Animals (Scientific Procedures) Act of 1986 (United Kingdom) or as approved by the German Regierungspräsidium Tübingen. The manuscript does not contain clinical studies or patient data.

Conflict of interest The authors declare that they have no conflict of interest.

Software availability The model and simulation experiments described herein have been conducted using free access software (NEURON, https://www.neuron.yale.edu/neuron/). Model parameters have been described extensively in the manuscript and model files are freely available from the authors upon request. Upon publication, the files will be made freely available to the modelDB database (https://senselab. med.yale.edu/modeldb/).

\section{Appendix}

\section{General model features}

General equations for the construction and the workings of the NEURON simulator and the implementation of neuronal model within NEURON have been described extensively previously (see Hines and Carnevale 1997) and are briefly discussed and presented below together with specific equations used for the working of vlPAG/DRN DA neuron.

To follow the time evolution of neuronal membrane potential $\left(V_{m}\right)$ for a neuron with a membrane capacitance $\mathrm{C}_{\mathrm{m}}$ one must obtain the sum of each operant ionic current (denoted as (ion)) flowing through ionic channels on the neuron's membrane. The general eq. (6) (current balance equation) below describes the relationship of membrane voltage and ionic current flowing through individual ionic channels for any neuron with membrane capacitance $\mathrm{C}_{\mathrm{m}}$.

$C m \frac{d V m}{d t}=-\sum I($ ion $)$

The ionic current $\left(\mathrm{I}_{(\mathrm{ion})}\right)$ passing through any ionic channel is both a voltage and time-dependent function. It can be calculated as the product of the ion's maximal conductance $\left(\mathrm{G}_{\max }\right)$ times the driving force at any given potential $\left(\mathrm{V}_{\mathrm{m}^{-}}\right.$ $E_{\text {rev, }}$, where $E_{\text {rev }}$ is the reversal potential for the conductance, Eq. (7))

$I($ ion $)=G \max (V m, t)^{*}(V m-$ Erev $)$

The ionic currents in the model were described by standard, Hodgkin-Huxley-type equations in which generalized Boltzmann-type equations defined the voltage- and timedependent activation and inactivation of conductances. Specifically, ionic conductances were evaluated by solving general eq. (8). Conductance $\left(\mathrm{G}_{(\mathrm{ion})}\right)$ of any given ion at any given time $t$ at any given $V_{m}$ can be described as the product of the ion's maximal conductance $\left(\mathrm{G}_{\max }\right)$ times the activation function $\left(\mathrm{A}_{(\mathrm{ion})}\right)$ times the inactivation function $\left(\mathrm{B}_{(\mathrm{ion})}\right)$ of the ion. $\mathrm{A}_{\text {(ion) }}\left(\mathrm{V}_{\mathrm{m}}, \mathrm{t}\right)$ and $\mathrm{B}_{\text {(ion) }}\left(\mathrm{V}_{\mathrm{m}}, \mathrm{t}\right)$ are functions describing the voltage and time dependence of activation and inactivation associated with $\mathrm{G}_{(\mathrm{ion})}$, while $\mathrm{p}$ and $\mathrm{n}$ is the power to which $\mathrm{A}_{(\mathrm{ion})}$ and $\mathrm{B}_{(\mathrm{ion})}$ were raised and was taken as the standard value for a given ionic channel. $P$ and $\mathrm{n}$ can be physically represented by the number of gating stages/steps for the ion channel activation and inactivation function respectively and were given a value of 3 and 1 for sodium channels and 4 and zero for potassium channel activation and inactivation functions respectively as described previously by Hodgkin and Huxley (1952).

$G($ ion $)=G \max (\text { ion })^{*} A^{p}($ ion $)(V m, t)^{*} B^{n}($ ion $)(V m, t)$

$\mathrm{A}_{(\mathrm{ion})}$ and $\mathrm{B}_{(\mathrm{ion})}$ were given by the solution to the general differential eqs. (9) and (10) below:

$\frac{d A(\text { ion })}{d t}=\frac{A \infty(\text { ion })-A(\text { ion })}{\tau A(\text { ion })}$

$\frac{d B(\text { ion })}{d t}=\frac{B \infty(\text { ion })-B(\text { ion })}{\tau B(\text { ion })}$

where $\mathrm{A}^{\mathbf{0 0}}{ }_{\text {(ion) }}$ and $\mathrm{B}^{\mathbf{0 0}}{ }_{\text {(ion) }}$ are the voltage-dependent, steady-state values of the activation and inactivation functions, respectively; and $\tau_{\mathrm{A} \text { (ion) }}$ and $\tau_{\mathrm{B} \text { (ion) }}$ are the voltage-dependent time constants of the activation and inactivation functions, 
respectively. The values of $\mathrm{A}^{\mathbf{0 0}}{ }_{\text {(ion) }}$ and $\mathrm{B}^{\mathbf{0 0}}{ }_{\text {(ion) }}$ were determined from the general eqs. (11) and (12) below:

$$
\begin{aligned}
& A \infty(\text { ion })=\frac{1}{1+\exp \left(\frac{V m-h A}{s A}\right)} \\
& B \infty(\text { ion })=\frac{1}{1+\exp \left(\frac{V m-h B}{s B}\right)}
\end{aligned}
$$

where $\mathrm{V}_{\mathrm{m}}$ is the membrane voltage, whereas $\mathrm{h}_{\mathrm{A}}, \mathrm{h}_{\mathrm{B})}$ and $\mathrm{s}_{\mathrm{A}}$, $\mathrm{s}_{\mathrm{B})}$ determine the midpoints $\left(\mathrm{V}_{50}\right)$ and slopes of the steady-state activation and inactivation functions, respectively. The voltage dependence of the activation and inactivation time constants ( $\tau_{\mathrm{A}(\text { ion) }}$ and $\left.\tau_{\mathrm{B}(\text { ion) }}\right)$ were represented by one of the two following functions respectively. Otherwise, $\tau$ was held constant.

$$
\begin{aligned}
& \tau \mathrm{A}(\text { ion })=\frac{\tau \mathrm{Amax}-\tau \mathrm{Amin}}{1+\exp \left(\frac{V m-h \tau A}{s \tau A}\right)}+\tau \mathrm{Amin} \\
& \tau B(\text { ion })=\frac{\tau B \max -\tau B \min }{1+\exp \left(\frac{V m-h \tau B}{s \tau B}\right)}+\tau B \min
\end{aligned}
$$

where $V_{m}$ is the membrane voltage; $\tau_{A \text { max }}, \tau_{A \text { min }}$ and $\tau_{B}$ ${ }_{\max }, \tau_{\mathrm{B} \min }$ are the maximal and minimal values for the activation and inactivation time constants, respectively; $h \tau_{\mathrm{A}}, \mathrm{h} \tau_{\mathrm{B}}$ and $\mathrm{s} \tau_{\mathrm{A}}, \mathrm{s} \tau_{\mathrm{B}}$ determine the $\left(\mathrm{V}_{50}\right)$ midpoints and slopes of the time constant functions, respectively. Equation (13) \& (14) were used since the voltage dependence of the time constants was best fit by a sigmoid in the physiological range.

Open Access This article is distributed under the terms of the Creative Commons Attribution 4.0 International License (http:// creativecommons.org/licenses/by/4.0/), which permits unrestricted use, distribution, and reproduction in any medium, provided you give appropriate credit to the original author(s) and the source, provide a link to the Creative Commons license, and indicate if changes were made.

\section{References}

Beier, K. T., Steinberg, E. E., DeLoach, K. E., Xie, S., Miyamichi, K., Schwarz, L., Gao, X. J., Kremer, E. J., Malenka, R. C., \& Luo, L. (2015). Circuit architecture of VTA dopamine neurons revealed by systematic input-output mapping. Cell, 162, 622-634.

Bishop, M. W., Chakraborty, S., Matthews, G. A., Dougalis, A., Wood, N. W., Festenstein, R., \& Ungless, M. A. (2010). Hyperexcitable substantia nigra dopamine neurons in PINK1- and HtrA2/Omideficient mice. Journal of Neurophysiology, 104, 3009-3020.

Branch, S. Y., Sharma, R., \& Beckstead, M. J. (2014) Aging decreases Ltype calcium channel currents and pacemaker firing fidelity in substantia nigra dopamine neurons. The Journal of neuroscience: the official journal of the Society for Neuroscience, 34, 9310-9318
Brevi, S., de Curtis, M., \& Magistretti, J. (2001). Pharmacological and biophysical characterization of voltage-gated calcium currents in the endopiriform nucleus of the guinea pig. Journal of Neurophysiology, 85, 2076-2087.

Brown, D. A., \& Adams, P. R. (1980) Muscarinic suppression of a novel voltage-sensitive $\mathrm{K}+$ current in a vertebrate neurone. Nature, 283, 673-676.

Brown, D. A., \& Passmore, G. M. (2009) Neural KCNQ (Kv7) channels. British Journal of Pharmacology, 156, 1185-1195.

Budde, T., Meuth, S., \& Pape, H. C. (2002). Calcium-dependent inactivation of neuronal calcium channels. Nature Reviews. Neuroscience, 3, 873-883.

Catterall, W. A. (2000). Structure and regulation of voltage-gated Ca2+ channels. Annual Review of Cell and Developmental Biology, 16, 521-555.

Chan, C. S., Guzman, J. N., Ilijic, E., Mercer, J. N., Rick, C., Tkatch, T., Meredith, G. E., \& Surmeier, D. J. (2007). Rejuvenation' protects neurons in mouse models of Parkinson's disease. Nature, 447, 1081-1086.

Deignan, J., Lujan, R., Bond, C., Riegel, A., Watanabe, M., Williams, J. T., Maylie, J., \& Adelman, J. P. (2012). SK2 and SK3 expression differentially affect firing frequency and precision in dopamine neurons. Neuroscience, 217, 67-76.

Ding, S., Wei, W., \& Zhou, F. M. (2011). Molecular and functional differences in voltage-activated sodium currents between GABA projection neurons and dopamine neurons in the substantia nigra. Journal of Neurophysiology, 106, 3019-3034.

Dougalis, A. G., Matthews, G. A., Bishop, M. W., Brischoux, F., Kobayashi, K., \& Ungless, M. A. (2012). Functional properties of dopamine neurons and co-expression of vasoactive intestinal polypeptide in the dorsal raphe nucleus and ventro-lateral periaqueductal grey. The European Journal of Neuroscience, 36, 3322-3332.

Drion, G., Bonjean, M., Waroux, O., Scuvée-Moreau, J., Liégeois, J. F., Sejnowski, T. J., Sepulchre, R., \& Seutin, V. (2010). M-type channels selectively control bursting in rat dopaminergic neurons. The European Journal of Neuroscience, 31, 827-835.

Drion, G., Massotte, L., Sepulchre, R., \& Seutin, V. (2011). How modeling can reconcile apparently discrepant experimental results: The case of pacemaking in dopaminergic neurons. PLoS Computational Biology, 7, e1002050.

Edwards, F. A., Konnerth, A., Sakmann, B., \& Takahashi, T. (1989). A thin slice preparation for patch clamp recordings from neurones of the mammalian central nervous system. Pflügers Archiv - European Journal of Physiology, 414, 600-612.

Flores, J. A., El Banoua, F., Galan-Rodriguez, B., \& Fernandez-Espejo, E. (2004). Opiate anti-nociception is attenuated following lesion of large dopamine neurons of the periaqueductal grey: Critical role for D1 (not D2) dopamine receptors. Pain, 110, 205-214.

Flores, J. A., Galan-Rodriguez, B., Ramiro-Fuentes, S., \& FernandezEspejo, E. (2006). Role for dopamine neurons of the rostral linear nucleus and periaqueductal gray in the rewarding and sensitizing properties of heroin. Neuropsychopharmacology : Official Publication of the American College of Neuropsychopharmacology, 31, 1475-1488.

Giannattasio, B., Jones, S. W., \& Scarpa, A. (1991). Calcium currents in the A7r5 smooth muscle-derived cell line. Calcium-Dependent and Voltage-Dependent Inactivation. The Journal of General Physiology, 98, 987-1003.

Grace, A. A., \& Bunney, B. S. (1983a). Intracellular and extracellular electrophysiology of nigral dopaminergic neurons-1. Identification and Characterization. Neuroscience, 10, 301-315.

Grace, A. A., \& Bunney, B. S. (1983b). Intracellular and extracellular electrophysiology of nigral dopaminergic neurons-2. Action Potential Generating Mechanisms and Morphological Correlates. Neuroscience, 10, 317-331. 
Grace, A. A., \& Bunney, B. S. (1984a). The control of firing pattern in nigral dopamine neurons: Burst firing. The Journal of Neuroscience : the Official Journal of the Society for Neuroscience, 4, 2877-2890.

Grace, A. A., \& Bunney, B. S. (1984b). The control of firing pattern in nigral dopamine neurons: Single spike firing. The Journal of Neuroscience : the Official Journal of the Society for Neuroscience, 4, 2866-2876.

Grace, A. A., \& Onn, S. P. (1989). Morphology and electrophysiological properties of immunocytochemically identified rat dopamine neurons recorded in vitro. The Journal of Neuroscience : the Official Journal of the Society for Neuroscience, 9, 3463-3481.

Grace, A. A., Bunney, B. S., Moore, H., \& Todd, C. L. (1997). Dopamine-cell depolarization block as a model for the therapeutic actions of antipsychotic drugs. Trends in Neurosciences, 20, 31-37.

Guzman, J. N., Sanchez-Padilla, J., Chan, C. S., \& Surmeier, D. J. (2009). Robust pacemaking in substantia nigra dopaminergic neurons. The Journal of neuroscience : the Official Journal of the Society for Neuroscience, 29, 11011-11019.

Guzman, J. N., Sanchez-Padilla, J., Wokosin, D., Kondapalli, J., Ilijic, E., Schumacker, P. T., \& Surmeier, D. J. (2010). Oxidant stress evoked by pacemaking in dopaminergic neurons is attenuated by DJ-1. Nature, 468, 696-700.

Haack, J. A., \& Rosenberg, R. L. (1994). Calcium-dependent inactivation of L-type calcium channels in planar lipid bilayers. Biophysical Journal, 66, 1051-1060.

Hadley, J. K., Noda, M., Selyanko, A. A., Wood, I. C., Abogadie, F. C., \& Brown, D. A. (2000). Differential tetraethylammonium sensitivity of KCNQ1-4 potassium channels. British Journal of Pharmacology, 129, 413-415.

Hansen, H. H., Ebbesen, C., Mathiesen, C., Weikop, P., Rønn, L. C., Waroux, O., Scuvée-Moreau, J., Seutin, V., \& Mikkelsen, J. D. (2006). The KCNQ channel opener retigabine inhibits the activity of mesencephalic dopaminergic systems of the rat. The Journal of Pharmacology and Experimental Therapeutics, 318, 1006-1019.

Hille, B. (2001). Ion channels of excitable membranes, pp. 95-131. Sunderland: Sinauer Associates Inc.

Hines, M. L., \& Carnevale, N. T. (1997). The NEURON simulation environment. Neural Computation, 9, 1179-1209.

Hodgkin, A. L., \& Huxley, A. F. (1952). A quantitative description of membrane current and its application to conduction and excitation in nerve. The Journal of Physiology, 117, 500-544.

Hokfelt, T., Martensson, R., Bjorklund, A., Kleinau, S., \& Goldstein, M. (1984). Distribution of tyrosine-hydroxylaseimmunoreactive neurons in the rat brain. In A. Bjorklund \& T. Hokfelt (Eds.), Handbook of chemical neuroanatomy (pp. 277379). Amsterdam: Elsevier Science.

Itri, J. N., Michel, S., Vansteensel, M. J., Meijer, J. H., \& Colwell, C. S. (2005) Fast delayed rectifier potassium current is required for circadian neural activity. Nature Neuroscience, 8, 650-656.

Jackson, A. C., Yao, G. L., \& Bean, B. P. (2004). Mechanism of spontaneous firing in dorsomedial suprachiasmatic nucleus neurons. The Journal of Neuroscience : the Official Journal of the Society for Neuroscience, 24, 7985-7998

Kang, Y., \& Kitai, S. T. (1993a). Calcium spike underlying rhythmic firing in dopaminergic neurons of the rat substantia nigra. Neuroscience Research, 18, 195-207.

Kang, Y., \& Kitai, S. T. (1993b). A whole cell patch-clamp study on the pacemaker potential in dopaminergic neurons of rat substantia nigra compacta. Neuroscience Research, 18, 209-221.

Keja, J. A., Stoof, J. C., \& Kits, K. S. (1992). Dopamine D2 receptor stimulation differentially affects voltage-activated calcium channels in rat pituitary melanotropic cells. The Journal of Physiology, 450, 409-435.

Khaliq, Z. M., \& Bean, B. P. (2008). Dynamic, nonlinear feedback regulation of slow pacemaking by A-type potassium current in ventral tegmental area neurons. The Journal of Neuroscience : the Official Journal of the Society for Neuroscience, 28, 10905-10917.

Khaliq, Z. M., \& Bean, B. P. (2010). Pacemaking in dopaminergic ventral tegmental area neurons: Depolarizing drive from background and voltage-dependent sodium conductances. The Journal of Neuroscience : the Official Journal of the Society for Neuroscience, 30, 7401-7413.

Kimm, T., \& Bean, B. P. (2014). Inhibition of A-type potassium current by the peptide toxin SNX-482. The Journal of Neuroscience : the Official Journal of the Society for Neuroscience, 34, 9182-9189.

Kimm, T., Khaliq, Z. M., \& Bean, B. P. (2015). Differential regulation of action potential shape and burst-frequency firing by BK and $\mathrm{Kv} 2$ channels in substantia Nigra dopaminergic neurons. The Journal of Neuroscience : the Official Journal of the Society for Neuroscience, $35,16404-16417$.

Koyama, S., \& Appel, S. B. (2006a). A-type K+ current of dopamine and GABA neurons in the ventral tegmental area. Journal of Neurophysiology, 96, 544-554.

Koyama, S., \& Appel, S. B. (2006b). Characterization of Mcurrent in ventral tegmental area dopamine neurons. Journal of Neurophysiology, 96, 535-543.

Koyama, S., Kanemitsu, Y., \& Weight, F. F. (2005). Spontaneous activity and properties of two types of principal neurons from the ventral tegmental area of rat. Journal of Neurophysiology, 93, 3282-3293.

Koyama, S., Brodie, M. S., \& Appel, S. B. (2007). Ethanol inhibition of m-current and ethanol-induced direct excitation of ventral tegmental area dopamine neurons. Journal of Neurophysiology, 97, 1977-1985.

Lacey, M. G., Mercuri, N. B., \& North, R. A. (1989). Two cell types in rat substantia nigra zona compacta distinguished by membrane properties and the actions of dopamine and opioids. The Journal of Neuroscience : the Official Journal of the Society for Neuroscience, 9, 1233-1241.

Lammel, S., Hetzel, A., Hackel, O., Jones, I., Liss, B., \& Roeper, J. (2008). Unique properties of mesoprefrontal neurons within a dual mesocorticolimbic dopamine system. Neuron, 57, 760-773.

Lammel, S., Ion, D. I., Roeper, J., \& Malenka, R. C. (2011). Projectionspecific modulation of dopamine neuron synapses by aversive and rewarding stimuli. Neuron, 70, 855-862.

Lerner, T. N., Shilyansky, C., Davidson, T. J., Evans, K. E., Beier, K. T., Zalocusky, K. A., Crow, A. K., Malenka, R. C., Luo, L., Tomer, R., \& Deisseroth, K. (2015). Intact-Brain analyses reveal distinct information carried by SNc dopamine Subcircuits. Cell, 162, 635-647.

Li, C., McCall, N. M., Lopez, A. J., \& Kash, T. L. (2013). Alcohol effects on synaptic transmission in periaqueductal gray dopamine neurons. Alcohol, 47, 279-287.

Li, C., Sugam, J. A., Lowery-Gionta, E. G., McElligott, Z. A., McCall, N. M., Lopez, A. J., McKlveen, J. M., Pleil, K. E., \& Kash, T. L. (2016). Mu opioid receptor modulation of dopamine neurons in the periaqueductal gray/dorsal raphe: A role in regulation of pain. Neuropsychopharmacology : Official Publication of the American College of Neuropsychopharmacology, 41, 2122-2132.

Liss, B., Franz, O., Sewing, S., Bruns, R., Neuhoff, H., \& Roeper, J. (2001). Tuning pacemaker frequency of individual dopaminergic neurons by Kv4.3L and KChip3.1 transcription. The EMBO Journal, 20, 5715-5724.

Lu, J., Jhou, T. C., \& Saper, C. B. (2006). Identification of wake-active dopaminergic neurons in the ventral periaqueductal gray matter. The Journal of neuroscience : the Official Journal of the Society for Neuroscience, 26, 193-202.

Magistretti, J., Castelli, L., Forti, L., \& D'Angelo, E. (2006). Kinetic and functional analysis of transient, persistent and resurgent sodium currents in rat cerebellar granule cells in situ: An electrophysiological and modelling study. The Journal of Physiology, 573, 83-106.

Malin, S. A., \& Nerbonne, J. M. (2002). Delayed rectifier K+ currents, IK, are encoded by Kv2 alpha-subunits and regulate tonic firing in 
mammalian sympathetic neurons. The Journal of Neuroscience : the Official Journal of the Society for Neuroscience, 22, 10094-10105.

Margolis, E. B., Lock, H., Hjelmstad, G. O., \& Fields, H. L. (2006). The ventral tegmental area revisited: Is there an electrophysiological marker for dopaminergic neurons? The Journal of Physiology, 577, 907-924.

Matthews, G. A., Nieh, E. H., Vander Weele, C. M., Halbert, S. A., Pradhan, R. V., Yosafat, A. S., Glober, G. F., Izadmehr, E. M., Thomas, R. E., Lacy, G. D., Wildes, C. P., Ungless, M. A., \& Tye, K. M. (2016). Dorsal raphe dopamine neurons represent the experience of social isolation. Cell, 164, 617-631.

Mercuri, N. B., Bonci, A., Calabresi, P., Stratta, F., Stefani, A., \& Bernardi, G. (1994). Effects of dihydropyridine calcium antagonists on rat midbrain dopaminergic neurones. British Journal of Pharmacology, 113, 831-838.

Milescu, L. S., Bean, B. P., \& Smith, J. C. (2010). Isolation of somatic $\mathrm{Na}+$ currents by selective inactivation of axonal channels with a voltage prepulse. The Journal of Neuroscience : the Official Journal of the Society for Neuroscience, 30, 7740-7748.

Mosharov, E. V., Larsen, K. E., Kanter, E., Phillips, K. A., Wilson, K., Schmitz, Y., Krantz, D. E., Kobayashi, K., Edwards, R. H., \& Sulzer, D. (2009). Interplay between cytosolic dopamine, calcium, and alpha-synuclein causes selective death of substantia nigra neurons. Neuron, 62, 218-229.

Nedergaard, S., Flatman, J. A., \& Engberg, I. (1993). Nifedipine- and omega-conotoxin-sensitive $\mathrm{Ca} 2+$ conductances in guinea-pig substantia nigra pars compacta neurones. The Journal of Physiology, 466, 727-747.

Neuhoff, H., Neu, A., Liss, B., \& Roeper, J. (2002). I(h) channels contribute to the different functional properties of identified dopaminergic subpopulations in the midbrain. The Journal of Neuroscience : the Official Journal of the Society for Neuroscience, 22, 1290-1302.

Philippart, F., Destreel, G., Merino-Sepúlveda, P., Henny, P., Engel, D., \& Seutin, V. (2016). Differential somatic $\mathrm{Ca} 2+$ channel profile in midbrain dopaminergic neurons. The Journal of Neuroscience : the Official Journal of the Society for Neuroscience, 36, 7234-7245.

Pignatelli, A., Kobayashi, K., Okano, H., \& Belluzzi, O. (2005). Functional properties of dopaminergic neurones in the mouse olfactory bulb. The Journal of Physiology, 564, 501-514.

Poetschke, C., Dragicevic, E., Duda, J., Benkert, J., Dougalis, A., DeZio, R., Snutch, T.P., Striessnig, J. \& Liss, B. (2015) Compensatory Ttype $\mathrm{Ca} 2+$ channel activity alters D2-autoreceptor responses of substantia nigra dopamine neurons from Cav1.3 L-type Ca2+ channel KO mice. Scientific reports, $\mathbf{5}, 13688$.

Pollack, A. E. (2001). Anatomy, physiology, and pharmacology of the basal ganglia. Neurologic Clinics, 19, 523-534.

Poulin, J. F., Zou, J., Drouin-Ouellet, J., Kim, K. Y., Cicchetti, F., \& Awatramani, R. B. (2014). Defining midbrain dopaminergic neuron diversity by single-cell gene expression profiling. Cell Reports, 9 , 930-943.

Puopolo, M., Raviola, E., \& Bean, B. P. (2007). Roles of subthreshold calcium current and sodium current in spontaneous firing of mouse midbrain dopamine neurons. The Journal of Neuroscience : the Official Journal of the Society for Neuroscience, 27, 645-656.

Putzier, I., Kullmann, P. H., Horn, J. P., \& Levitan, E. S. (2009). Cav1.3 channel voltage dependence, not $\mathrm{Ca} 2+$ selectivity, drives pacemaker activity and amplifies bursts in nigral dopamine neurons. The Journal of Neuroscience : the Official Journal of the Society for Neuroscience, 29, 15414-15419.
Schultz, W. (1998). Predictive reward signal of dopamine neurons. Journal of Neurophysiology, 80, 1-27.

Seutin, V., Massotte, L., Renette, M. F., \& Dresse, A. (2001). Evidence for a modulatory role of $\mathrm{Ih}$ on the firing of a subgroup of midbrain dopamine neurons. Neuroreport, 12, 255-258.

Shah, M. M., Migliore, M., \& Brown, D. A. (2011). Differential effects of Kv7 (M-) channels on synaptic integration in distinct subcellular compartments of rat hippocampal pyramidal neurons. The Journal of Physiology, 589, 6029-6038.

Silva, N. L., Pechura, C. M., \& Barker, J. L. (1990). Postnatal rat nigrostriatal dopaminergic neurons exhibit five types of potassium conductances. Journal of Neurophysiology, 64, 262-272.

Stuart, G. J., Dodt, H. U., \& Sakmann, B. (1993). Patch-clamp recordings from the soma and dendrites of neurons in brain slices using infrared video microscopy. Pflügers Archiv - European Journal of Physiology, 423, 511-518.

Subramaniam, M., Althof, D., Gispert, S., Schwenk, J., Auburger, G., Kulik, A., Fakler, B.\& Roeper, J. (2014) Mutant $\alpha$-synuclein enhances firing frequencies in dopamine substantia nigra neurons by oxidative impairment of A-type potassium channels. The Journal of Neuroscience : the Official Journal of the Society for Neuroscience, 34, 13586-13599.

Surmeier, D. J., \& Schumacker, P. T. (2013). Calcium, bioenergetics, and neuronal vulnerability in Parkinson's disease. The Journal of Biological Chemistry, 288, 10736-10741.

Surmeier, D. J., Guzman, J. N., Sanchez-Padilla, J., \& Schumacker, P. T. (2011). The role of calcium and mitochondrial oxidant stress in the loss of substantia nigra pars compacta dopaminergic neurons in Parkinson's disease. Neuroscience, 198, 221-231.

Tucker, K. R., Huertas, M. A., Horn, J. P., Canavier, C. C., \& Levitan, E. S. (2012). Pacemaker rate and depolarization block in nigral dopamine neurons: A somatic sodium channel balancing act. The Journal of Neuroscience : The Official Journal of the Society for Neuroscience, $32,14519-14531$.

Wilson, C. J., \& Callaway, J. C. (2000). Coupled oscillator model of the dopaminergic neuron of the substantia nigra. Journal of Neurophysiology, 83, 3084-3100.

Wladyka, C. L., \& Kunze, D. L. (2006). KCNQ/M-currents contribute to the resting membrane potential in rat visceral sensory neurons. The Journal of Physiology, 575, 175-189.

Wolfart, J., \& Roeper, J. (2002). Selective coupling of T-type calcium channels to SK potassium channels prevents intrinsic bursting in dopaminergic midbrain neurons. The Journal of Neuroscience : the Official Journal of the Society for Neuroscience, 22, 3404-3413.

Wolfart, J., Neuhoff, H., Franz, O., \& Roeper, J. (2001). Differential expression of the small-conductance, calcium-activated potassium channel SK3 is critical for pacemaker control in dopaminergic midbrain neurons. The Journal of Neuroscience : the Official Journal of the Society for Neuroscience, 21, 3443-3456.

Xiao, J., Cai, Y., Yen, J., Steffen, M., Baxter, D. A., Feigenspan, A., \& Marshak, D. (2004). Voltage-clamp analysis and computational model of dopaminergic neurons from mouse retina. Visual Neuroscience, 21, 835-849.

Zhao, S., Maxwell, S., Jimenez-Beristain, A., Vives, J., Kuehner, E., Zhao, J., O'Brien, C., de Felipe, C., Semina, E., \& Li, M. (2004). Generation of embryonic stem cells and transgenic mice expressing green fluorescence protein in midbrain dopaminergic neurons. The European Journal of Neuroscience, 19, 1133-1140. 